듬 SCHOOL of GRADUATE STUDIES

EAST TENNESSEE STATE UNIVERSITY
East Tennessee State University Digital Commons@ East Tennessee State University

$5-2014$

\title{
Assessment of Red Blood Cell Membrane Fatty Acid Composition in Relation to Dietary Intake in Patients Undergoing Cardiac Catheterization
}

Nicole S. Litwin

East Tennessee State University

Follow this and additional works at: https://dc.etsu.edu/etd

Part of the Dietetics and Clinical Nutrition Commons

\section{Recommended Citation}

Litwin, Nicole S., "Assessment of Red Blood Cell Membrane Fatty Acid Composition in Relation to Dietary Intake in Patients Undergoing Cardiac Catheterization" (2014). Electronic Theses and Dissertations. Paper 2319. https://dc.etsu.edu/etd/2319

This Thesis - Open Access is brought to you for free and open access by the Student Works at Digital Commons @ East Tennessee State University. It has been accepted for inclusion in Electronic Theses and Dissertations by an authorized administrator of Digital Commons @ East Tennessee State

University. For more information, please contact digilib@etsu.edu. 
Assessment of Red Blood Cell Membrane Fatty Acid Composition in Relation to Dietary Intake in Patients Undergoing Cardiac Catheterization

\begin{tabular}{c} 
A thesis \\
presented to \\
the faculty of the Department of Allied Health Sciences \\
East Tennessee State University \\
In partial fulfillment \\
of the requirements for the degree \\
Master of Science in Clinical Nutrition \\
by \\
Nicole Litwin \\
May 2014 \\
Eileen Cress \\
W. Andrew Clark, Chair \\
\hline
\end{tabular}

Keywords: red blood cell membrane, fatty acid composition, omega-3 fatty acids, coronary artery disease, oxidative stress 


\begin{abstract}
Assessment of Red Blood Cell Membrane Fatty Acid Composition in Relation to Dietary Intake in Patients Undergoing Cardiac Catheterization

by

Nicole Litwin
\end{abstract}

Red blood cells (RBC) have been shown to mediate plaque development seen in coronary artery disease (CAD). This study determined whether differences in RBC fatty acid (FA) composition were related to CAD risk. FAs were extracted from RBCs of 38 individuals who have undergone cardiac catheterization, 9 of whom had obstructive CAD, and analyzed via gas chromatography. Ferric reducing ability of plasma (FRAP) assay was used to determine oxidative stress. Food frequency questionnaires were used to correlate RBC omega-3 FA to daily intake of omega-3 FA. No correlation was found between RBC content and intake of omega-3 FA. FRAP values and RBC FA composition did not differ between the 2 groups with exception of the saturated FA, palmitic acid ( $p=0.018)$. These results suggest that RBC FA composition may differ between individuals with or at risk for CAD. Additional research is needed to validate this biomarker as a predictor of CAD. 
Copyright 2013 by Nicole Litwin, All Rights Reserved 


\section{ACKNOWLEDGEMENTS}

First, I would like to thank Dr. Balraj Singh and Dr. Kamesh Sivagnanam for recruiting study participants at the ETSU Heart Clinic and all clinic personnel who assisted with blood withdrawal. I would like to thank Elizabeth Sublette for allowing the use of her food frequency questionnaire for inclusion in this study. I would also like to thank ETSU graduate student and dietetic intern, Robert Santos-Prowse, for assisting with the methylation process of blood samples. And lastly, I would like to thank my thesis chair and committee members for all their time and support during my research process. This research was supported by a thesis research grant and thesis scholarship awarded by ETSU School of Graduate Studies. 


\section{TABLE OF CONTENTS}

Page

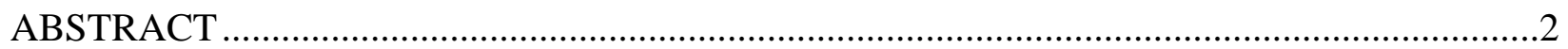

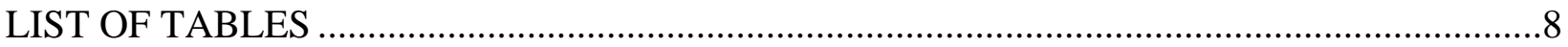

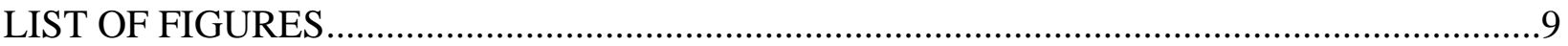

\section{Chapter}

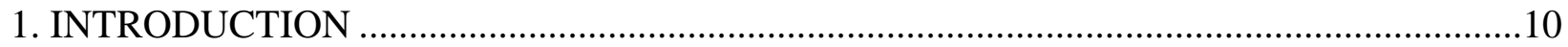

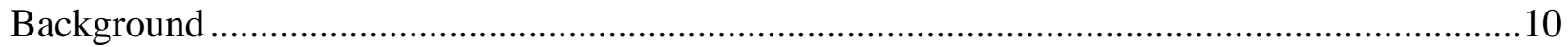

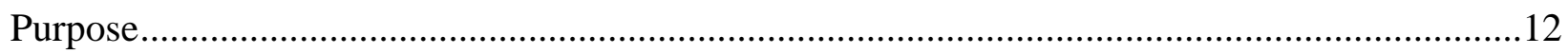

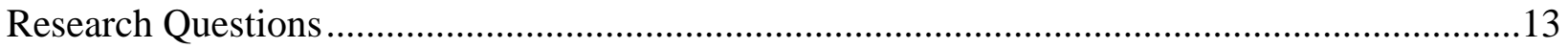

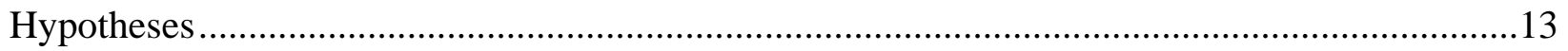

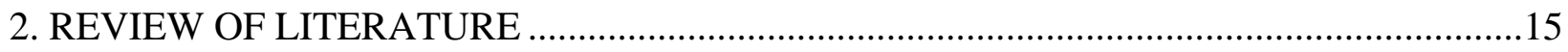

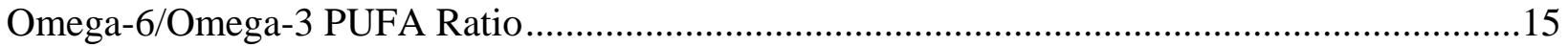

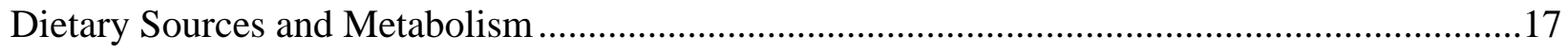

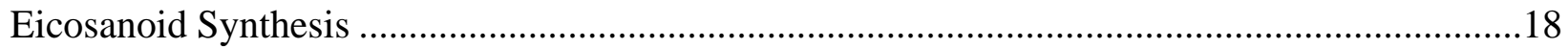

Cardiovascular Effects of Omega-3 Fatty Acids: CHD Evidence Explained..............................20

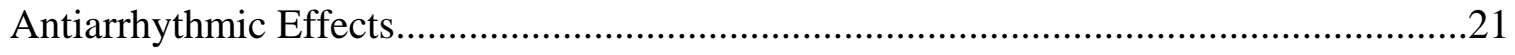

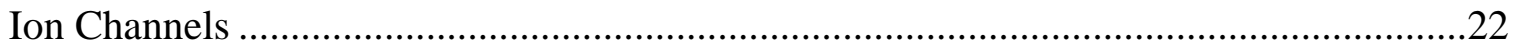

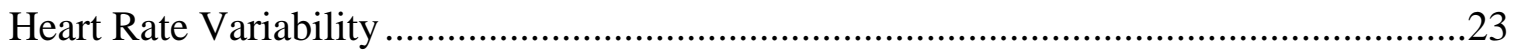

Prevention of Sudden Cardiac Death ......................................................................24

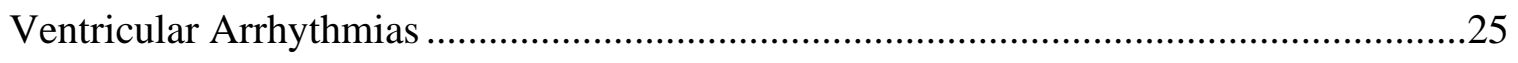

Intermediate Chain Omega-3 Fatty Acids and Ventricular Arrhythmias ........................27 
Coronary Artery Bypass Graft

Anti-Inflammatory Effects and Atherosclerotic Plaque Progression and Vulnerability ..... .30

PUFA Incorporation into Atherosclerotic Plaque. .32

Clinical Implications .35

Red Blood Cell Membrane Fatty Acid Composition....................................................37

Red Blood Cell Fatty Acid Patterns and Acute Coronary Syndrome..............................37

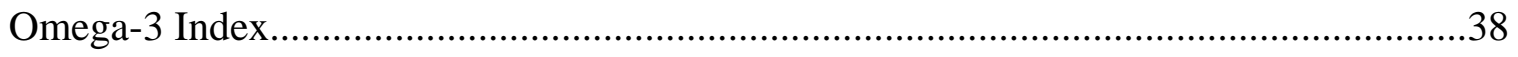

Factors Influencing Red Blood Cell Fatty Acid Composition......................................40

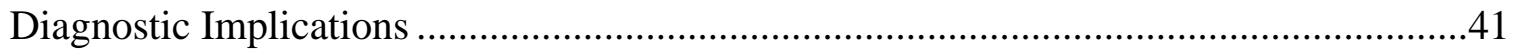

Food Frequency Questionnaire: Validity and Reliability ....................................................42

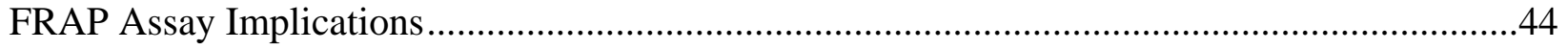

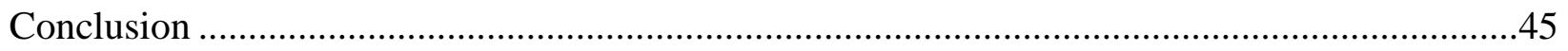

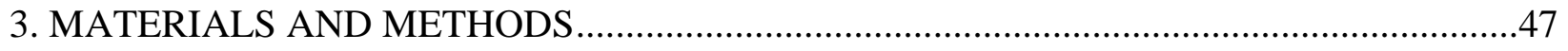

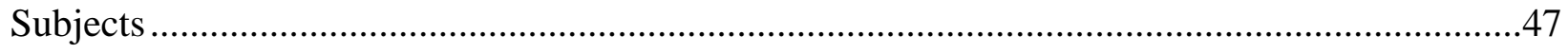

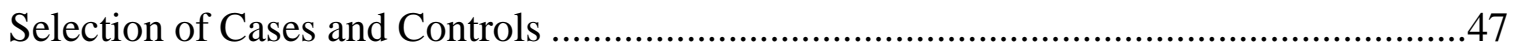

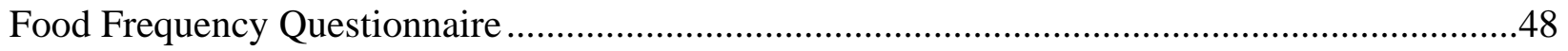

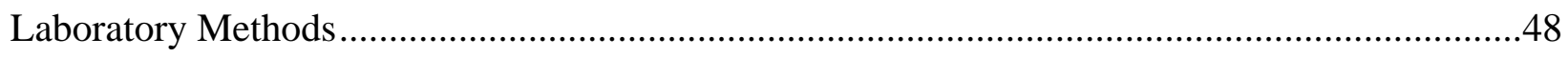

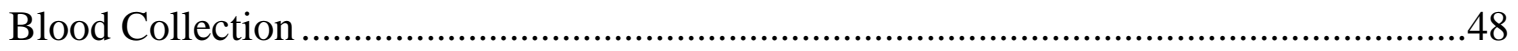

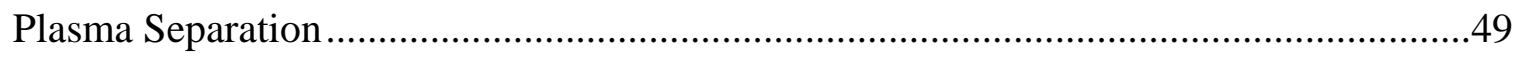

Isolation of Red Blood Cells and Extraction of Lipids..............................................49

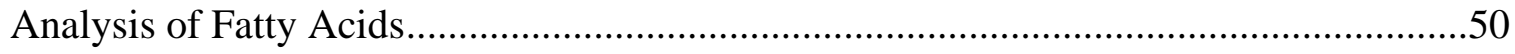

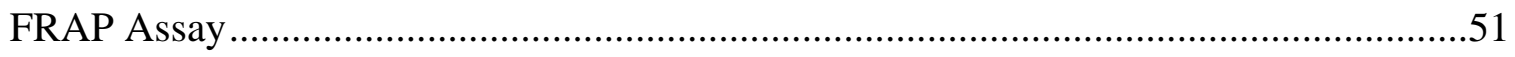

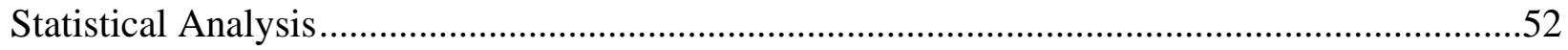




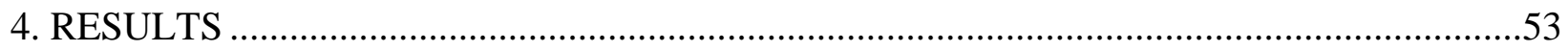

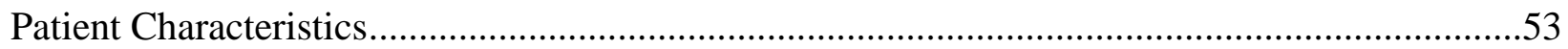

Red Blood Cell Fatty Acid Composition .......................................................................................56

Food Frequency Questionnaire Responses .................................................................................

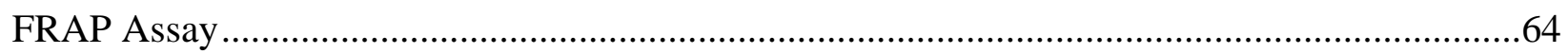

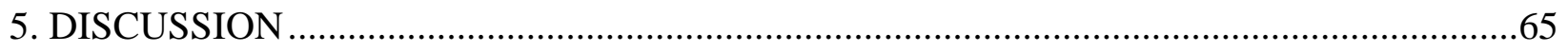

Red Blood Cell Fatty Acids and Dietary Intake …………...........................................................65

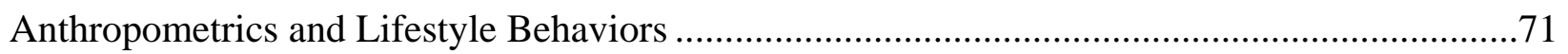

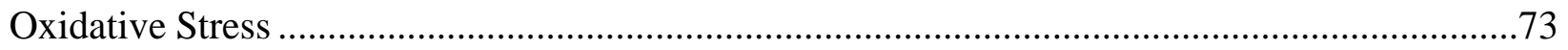

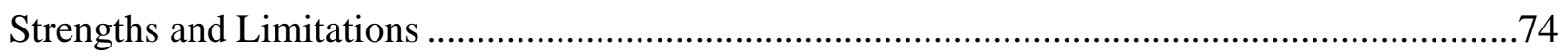

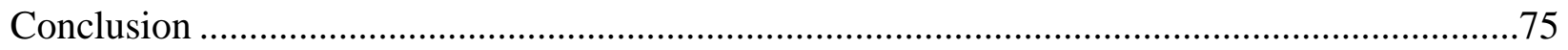

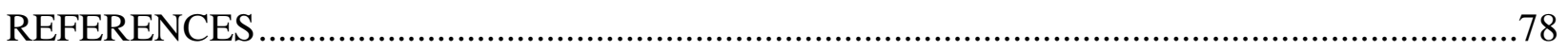

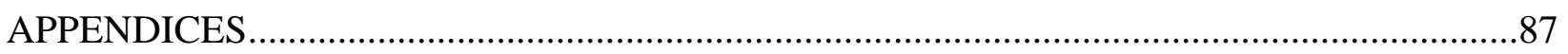

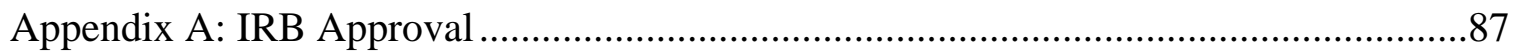

Appendix B: Permission Letter to Use Food Frequency Questionnaire..............................89

Appendix C: Omega-3 Fatty Acid Food Frequency Questionnaire.......................................90

Appendix D: Results from General Linear Models ............................................................94

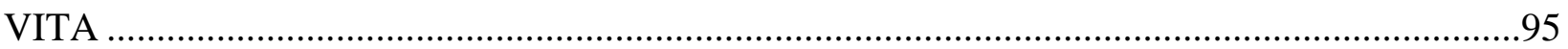




\section{LIST OF TABLES}

Table

Page

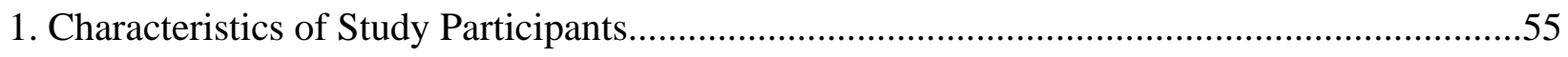

2. Fatty Acid Profiles of RBC Membranes for Cases and Controls............................................57

3. Average ALA, EPA, DHA Consumed as Reported in FFQ ................................................59 


\section{LIST OF FIGURES}

Figure

1. Average RBC palmitic acid content by case status .............................................................58

2. Average RBC omega-6 fatty acid level by case status......................................................58

3. Correlation between average RBC omega-3 content and average FFQ omega-3 consumption

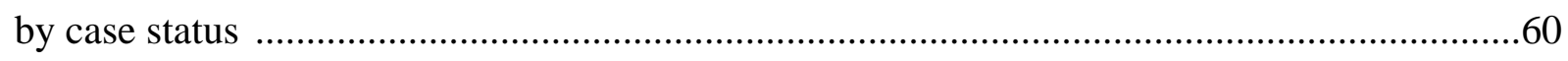

4. Fish consumption reported in FFQ for case and control status.........................................60

5. Frequency of fish consumption in past 6 months reported in FFQ by case and control status..61

6. Walnut and flaxseed consumption reported in FFQ for case and control status 61

7. Frequency of walnut consumption in past 6 months reported in FFQ for case and control status

8. Canola oil, flaxseed oil, and cod liver oil consumption reported in FFQ for case and control status

9. Frequency of canola oil consumption in past 6 months reported in FFQ by case and control status

10. Omega-3 supplement usage reported in FFQ by case and control status.

11. Average FRAP value by case and control status .64 


\section{CHAPTER 1}

\section{INTRODUCTION}

\section{Background}

Coronary artery disease (CAD), also called coronary heart disease (CHD) and is used interchangeably hereafter, is a general term that describes all causes of heart disease characterized by narrowing of blood vessels that supply the heart. ${ }^{1} \mathrm{CAD}$ is currently the leading cause of death and morbidity for both men and women worldwide. ${ }^{1}$ CAD occurs when plaque accumulates in coronary arteries and is primarily caused by atherosclerosis, which is described as the development of atherosclerotic plaque in the vascular wall that accrues and produces ischemic conditions by insufficient blood flow or by rupturing, forming a thrombus, and occluding the lumen of the vessel. ${ }^{1}$ Atherosclerotic plaque can be described as unstable or vulnerable, suggesting a high risk of rupture that leads to thrombus formation, thus increasing heart attack and stroke risk. ${ }^{1}$ Vulnerable plaque is characterized by lesions with a thin, fibrous cap, few smooth muscle cells, macrophages, and a large lipid core that is composed of cholesterol, lipids, and dead foam cell remnants. ${ }^{1,2}$

Instability of plaque is dependent upon the thinning of its fibrous cap, enlargement of the lipid core, and high inflammatory content from macrophage infiltration ${ }^{2-4}$, all of which increase the risk of intraplaque hemorrhage and rupture. ${ }^{3,4}$ When intraplaque hemorrhage occurs, red blood cells (RBC) enter the atherosclerotic lipid core of the plaque, which results in an increase of cholesterol accumulation due to the large amounts of (free) cholesterol contained in RBC membranes. ${ }^{2,3,4} \mathrm{RBC}$ membranes are 1.5-2.0 times richer in cholesterol than any other cell in the human body and approximately $40 \%$ of its cell weight is composed of lipids. ${ }^{2}$ Cholesterol 
concentration in RBC membranes reflects lipid profile over a long period of time; therefore, it is plausible that the cholesterol content of RBC membranes is an index of plasma cholesterol concentrations reflective of the lipids consumed over the last several months. ${ }^{2}$ The RBC with its lipid-rich membrane can contribute to the cholesterol found in atherosclerotic plaque due to the high cholesterol content exceeding that of all other cells. ${ }^{2}$ Thus, it can be postulated that most of the free cholesterol found in atherosclerotic plaque that can lead to plaque instability in CAD is derived from the RBC membrane. ${ }^{2}$

Moreover, atherosclerotic plaque growth and stability/instability depends on plasma lipid composition determined by dietary fatty acid intake. ${ }^{5}$ It is well known that consumption of different dietary fatty acids influences the risk and progression of cardiovascular diseases. Numerous epidemiological studies have shown that high intakes of saturated fatty acids are associated with an increased risk of CHD, while increased consumption of polyunsaturated fatty acids (PUFA) are associated with a reduced risk and CHD protection. ${ }^{4,5}$ Recently, it has been acknowledged that distinguishing the lipid composition of RBC membranes can be used as an additional risk factor for CHD. ${ }^{6}$ The RBC membrane fatty acid composition is becoming more preferred than plasma fatty acids because RBC fatty acids reveal long-term dietary fats consumed and is not affected by recent dietary fat intake. ${ }^{6,7}$

The analysis of RBC membrane fatty acid composition is essential in establishing a patient's RBC fatty acid profile in relationship to CAD risk based on dietary patterns. The RBC fatty acid profile will indicate saturated, polyunsaturated, and monounsaturated fatty acid concentrations that can be used to determine ratios between saturated vs. unsaturated fats, MUFA vs. PUFA and omega-6 PUFA vs. omega-3 PUFA. The amounts of omega-6 and omega3 PUFA in the RBC membrane are particularly important due to their synthesis of eicosanoids. ${ }^{8}$ 
For instance, a high amount of omega-6 PUFA in the diet shifts the body's physiological state to one that exhibits characteristics of its respective eicosanoids, resulting in increases of blood viscosity, vasospasms, and vasoconstriction. ${ }^{8}$ On the other hand, increasing omega-3 fatty acids in the diet produces higher amounts of eicosanoids that are less potent inducers of inflammation, blood vessel constriction, and coagulation, thus providing cardiovascular protection. ${ }^{8}$

Determining the fatty acid composition of the RBC membrane can establish a patient's typical lipid pattern based on dietary intake and can be used as a complementary diagnostic tool for monitoring the progression of CAD in a safe, noninvasive manner to help detect imbalances of fatty acids. This novel, diagnostic tool can aid in the selection and evaluation of dietary intervention therapies in clinical settings.

\section{Purpose}

The purpose of this study was to determine if differences in RBC membrane fatty acid composition are correlated with CAD and cardiac conditions such as coronary artery bypass grafts and coronary artery stents that are commonly used to treat CAD. The RBC membranes of these patients contain a fatty acid composition indicative of the typical lipids consumed during the lifespan of the RBC, which is usually about 3 - 4 months. Evidence has shown that RBCs play an important role in the development of atherosclerotic plaque seen in CAD. Instability of atherosclerotic plaque is driven by the accumulation of cholesterol derived from RBC membranes' high cholesterol content determined in part by plasma lipid composition, which is influenced by dietary intake. Because RBC fatty acid composition reflects long-term lipid consumption, discovering a significant difference between case and control groups may indicate a role for the analysis of RBC membrane in evaluating predictive risks of CAD. It is our intent to 
use this tool in future experiments to determine if we can identify patients ( $<30$ years of age) who are at risk and may benefit from dietary intervention (i.e. early changes in diet to avoid cardiac procedures to correct blockages).

\section{$\underline{\text { Research Questions }}$}

1. Do patients with CAD have different RBC membrane fatty acid composition and dietary patterns (based on food frequency questionnaire results) than age-matched patients who have had a cardiac catheterization but without any identified blockages?

2. What are the characteristic body mass index (BMI), age, sex, family history, and other anthropometrics/demographics of patients with blockages and those not showing a blockage after cardiac catheterization?

3. Will level of oxidative stress differ between patients with blockages and those not showing a blockage after cardiac catheterization?

\section{Hypotheses}

Hypothesis (1): There will be a significant difference in the RBC fatty acid composition and dietary consumption of omega-3 PUFA between patients who had blockages identified during cardiac catheterization and those who did not have blockages identified. 
Hypothesis (2): The level of oxidative stress will be significantly different between patients who had blockages identified during cardiac catheterization and those who did not have blockages identified. 


\section{CHAPTER 2}

\section{REVIEW OF LITERATURE}

In recent years, extensive research has been conducted on the metabolism of polyunsaturated fatty acids (PUFA), particularly omega-6 and omega-3 fatty acids, due to their essentiality in the human diet. Long-chain omega-6 and omega-3 PUFA are considered essential fatty acids (EFA) because humans, like all mammals, cannot synthesize them within the body, nor are the fats interconvertible; therefore, they must be obtained through the diet. ${ }^{8}$ The physiological effects of PUFA are distinct from the more abundant saturated and monounsaturated fatty acids (MUFA) found in today’s Western diet. Saturated and monounsaturated fatty acids can be synthesized in mammalian tissues from glucose or amino acid precursors. ${ }^{10}$ However, this does not typically occur in humans consuming a Western diet because the majority of dietary fat intake is from saturated, monounsaturated, and trans fatty acids. ${ }^{10}$ Fatty acids in general provide a source of energy, while only a proportion of EFA are used as an energy source due to their biological functions that include stimulation of growth, maintenance of skin and hair growth, regulation of cholesterol metabolism, lipotropic activity, maintenance of reproductive performance, and other physiologic and pharmacologic effects. ${ }^{11}$ EFA also help maintain membrane integrity and an optimal level of unsaturation in tissue lipids. $^{11}$

\section{Omega-6/Omega-3 PUFA Ratio}

In the Western diet consumption of PUFA contributes about 7\% of total energy intake and $19-22 \%$ of energy intake from fat in the average adult diet. ${ }^{12}$ Although this is within the recommended intake for men and women, consumption of omega-6 fatty acids greatly exceeds 
that of omega-3 fatty acids. ${ }^{12}$ This is of great importance due to the role that omega-3 fatty acids play in normal growth and development of several organs (e.g. heart, brain, reproductive organs) and the prevention and management of coronary heart disease, hypertension, type 2 diabetes, arthritis, other inflammatory and autoimmune diseases, and cancer. ${ }^{9}$ In addition, the ratio of omega-6 and omega-3 fatty acids in the Western diet is notably different from the fatty acid profile and overall fat content seen during prehistoric times. Based on estimates from studies of Paleolithic nutrition (40,000 - 45,000 years ago), a time in which humans evolved and genetic profiles were established, it is apparent that humans evolved consuming a diet that is significantly lower in saturated and trans fatty acids than today's modern diet. ${ }^{8}$ The early human diet contained equal quantities of omega-6 to omega-3 fatty acids (ratio of 1:1), whereas the current Western diet contains excessive amounts of omega-6 fatty acids and almost deficient amounts of omega-3 fatty acids. For example, today's ratio of omega-6 to omega 3 fatty acids (n6: $\mathrm{n}-3$ ) is found to be at 15-30:1 or as much as 100:1. ${ }^{8}$ This marked shift in the ratio of the 2 EFA is due to a decrease in fish consumption and industrial production of cereal feeds for livestock that are rich in omega-6 fatty acids and poor in omega-3 fatty acids, thereby leading to production of meat rich in omega-6 PUFA and poor in omega-3 PUFA. The same is also true for eggs, vegetable oil, cultivated vegetables, and cultured fish. ${ }^{8}$ Due to industrialization, omega-6 fatty acid consumption increased at the expense of omega-3 fatty acids. ${ }^{8,12}$ This imbalance of omega-6 to omega-3 fatty acids represents excessive amounts of omega-6 PUFA (i.e. high n-6: $\mathrm{n}$-3) that can promote the pathogenesis of several chronic diseases including cardiovascular disease, obesity, cancer, and autoimmune and inflammatory diseases. ${ }^{8}$ On the other hand, increased amounts of omega-3 PUFA in the diet (i.e. low n-6: n-3) can promote a suppressive 
effect against chronic diseases. ${ }^{8}$ The optimal ratio of n-6: n-3 in the diet is thought to be about $4: 1 .^{8}$

\section{Dietary Sources and Metabolism}

Currently, linoleic acid (LA; 18:2n-6) is the major PUFA consumed in the United States, compromising $84-89 \%$ of total PUFA energy intake in diets of the adult population. ${ }^{12}$ Omega-6 PUFA are represented by linoleic acid that can be found in vegetable oils such as corn, safflower, peanuts, and soybeans. Omega-3 PUFA are represented by $\alpha$-linolenic acid (ALA, 18:3 n-3) that can be found in flaxseed and flaxseed (linseed) oil, chia seed, perilla seed, walnuts, and canola (rapeseed) oil. ${ }^{8}$ The intake of omega-3 fatty acids in the U.S. is about $1.6 \mathrm{~g} / \mathrm{d}$ or $0.7 \%$ of total PUFA energy intake. ${ }^{12}$ Of this, ALA accounts for $1.4 \mathrm{~g} / \mathrm{d}$, while only $0.1-0.2 \mathrm{~g} / \mathrm{d}$ comes from EPA (20:5n-3) and DHA (22:6n-3) ${ }^{12,13}$ EPA and DHA are predominantly found in oily fish (e.g. mackerel, tuna, halibut, herring, salmon) and commercial fish oil products that are prepared from processing body fat of oily fish. ${ }^{10,13}$ EPA and DHA are also found in high proportions in the oils extracted from liver of other fish species such as cod. ${ }^{10}$ However, the current average daily intake of EPA and DHA combined in a typical Western diet is only about one fish serving every 10 days, which is approximately $0.15 \%$ of total dietary fat intake. ${ }^{14}$ On the other hand, major sources of ALA in the U.S. diet appear to be from vegetable oils, primarily canola and soybean oils that are greatly enriched with both omega- 6 and omega- 3 fatty acids. ${ }^{12,14}$ Although some amount of ALA can be further metabolized to longer chain fatty acids such as DHA and EPA after ingestion, this remains to be minimal $(<5 \%)$ and controversial. ${ }^{13,14}$ Similarly, LA can be converted into a longer chain fatty acid metabolite, arachidonic acid (AA; 20:4n-6). Both conversions depend on the concentration of omega- 6 and omega- 3 fatty acids in the diet and may be further limited by aging and certain disease conditions. ${ }^{14}$ 
LA, ALA, and their long-chain, highly unsaturated derivatives are important structural components of practically all cell membranes within the human body. ${ }^{9}$ After ingestion, LA and ALA are selectively distributed between triglycerides, adipose tissue stores, and tissue structural lipids. ${ }^{8}$ PUFA are oxidized more rapidly after ingestion compared to saturated and monounsaturated fatty acids. However, the long-chain PUFA derived from LA and ALA are spared from oxidation. ${ }^{11}$ These fatty acids, if preformed before becoming present in the diet, are incorporated into phospholipids of tissues almost 20 times more efficiently than they are incorporated after synthesis from dietary LA and ALA. ${ }^{11}$ When ingested EPA and DHA from dietary sources replaces omega- 6 fatty acids, especially AA, in the membranes of almost all cells, but particularly in the membranes of platelets, erythrocytes, neutrophils, monocytes, and hepatocytes. ${ }^{8}$ Therefore, the PUFA composition of cell membranes is greatly dependent on dietary intake.

\section{Eicosanoid Synthesis}

The respective derivatives of LA and ALA can be metabolized into bioactive eicosanoids such as luekotrienes, prostaglandins, thromboxanes, lipoxins, and other hydroxy fatty acids. ${ }^{8,14}$ Eicosanoids derived from AA are proinflammatory, proaggregatory, and prothrombotic, whereas eicosanoids derived from EPA are anti-inflammatory and inhibit platelet aggregation. ${ }^{8,14}$ With that being said, the ratio of omega- 6 to omega-3 fatty acids, or more specifically the absolute amounts of omega-3 fatty acids in the diet, may be crucial in the prevention of multiple disease states. ${ }^{8}$ This is due to competition between the 2 essential fatty acids for elongation/desaturation pathways for production of their respective derivatives, thereby resulting in increased synthesis of their eicosanoids. ${ }^{8,14}$ Additionally, a high intake of dietary LA interferes with the desaturation and elongation of ALA, while excessive amounts of dietary trans 
fatty acids interferes with the desaturation and elongation of both ALA and LA. ${ }^{8}$ As a result the relative amount and availability of AA, EPA, and DHA is diminished for further metabolism. ${ }^{8}$

Due to the excessive amounts of omega-6 fatty acids in the Western diet, the body's physiological state shifts to one that exhibits the characteristics of AA-derived eicosanoids that increase blood viscosity, vasospasms, and vasoconstriction and decrease bleeding time. ${ }^{8}$

Eicosanoid metabolic products from AA are biologically active in small quantities, but if formed in large amounts, they contribute to the formation of thrombi and atheromas, which indicates that dietary AA and LA can increase the risk of cardiovascular disease in individuals who consume excessive amounts of omega- 6 fatty acids. ${ }^{8,9}$ AA is typically the principal precursor for eicosanoid synthesis because the membranes of most cells contain larger amounts of AA when compared to LA and EPA. ${ }^{10}$ Various phospholipase enzymes, primarily phospholipase $\mathrm{A}_{2}$, can release AA in cell membranes and subsequently leave free AA to act as a substrate for cyclooxygenase-2 (COX-2) after stimulation of platelets and endothelial cells. ${ }^{15} \mathrm{COX}-2$ stimulates thromboxane $A_{2}$, a potent promoter of platelet aggregation, and prostacyclin $I_{2}$ $\left(\mathrm{PGI}_{2}\right)$, a potent inhibitor of platelet aggregation. ${ }^{15}$ Free AA can also act as a substrate for lipoxygenase enzymes that form 4-series leukotrienes (LT) that regulate the production of proinflammatory cytokines. For example, LT-B 4 enhances production of tumor necrosis factor (TNF)- $\alpha$, interleukin (IL)-1, IL-6, and interferon (IFN)- $\gamma$. So, overall, AA gives rise to a range of mediators some of which have opposing effects, but the general physiological effect is governed by the concentration of those mediators, timing of production, and sensitivities of target cells to their potent effects. ${ }^{10}$

The amount of AA in membrane phospholipids of platelets and endothelial cells can be reduced by distinctive mechanisms of long-chain omega-3 fatty acids, especially EPA. ${ }^{15}$ 
Increased availability of omega-3 fatty acids from regular fish consumption or fish oil supplementation would thereby result in a decreased amount of substrate available for synthesis of eicosanoids from AA. This would also result in a decreased production and concentration of thromboxane $\mathrm{A}_{2}$ (inducer of platelet aggregation and vasoconstriction) and proinflammatory cytokines produced by $\mathrm{LTB}_{4}{ }^{15} \mathrm{EPA} / \mathrm{DHA}$ can also be released from cell membrane phospholipids by the action of phospholipase $\mathrm{A}_{2}$ and also act as a substrate for COX-2. However, the products produced, 5-series leukotrienes $\left(\mathrm{LTB}_{5}\right.$ ), thromboxane $\mathrm{A}_{3}$, and prostacyclin $\mathrm{PGI}_{3}$, have a different structure and potency from those produced from AA. ${ }^{15}$ For instance, $\mathrm{TXA}_{3}$ is a weaker platelet aggregator and vasoconstrictor, and $\mathrm{LTB}_{5}$ is a weaker inducer of inflammation. ${ }^{9,15} \mathrm{EPA}$ also leads to increased concentrations of $\mathrm{PGI}_{3}$, leading to an overall increase in total prostacyclin by increasing $\mathrm{PGI}_{3}$ without decreasing $\mathrm{PGI}_{2}$ concentrations. ${ }^{9}$ This is of particular importance because both $\mathrm{PGI}_{2}$ and $\mathrm{PGI}_{3}$ are active vasodilators and inhibitors of platelet aggregation. Overall, EPA suppresses the production of AA-derived eicosanoids while increasing the production of EPA-derived eicosanoids that are less potent inducers of inflammation, blood vessel constriction, and coagulation, and thus promoting a less thrombotic environment that offers cardiovascular protection. ${ }^{10,14,15}$

\section{Cardiovascular Effects of Omega-3 Fatty Acids: CHD Evidence Explained}

Furthermore, several lines of evidence (epidemiological, mechanistic, and clinical trials) support that omega-3 PUFA, particularly EPA and DHA, exhibit cardio-protective effects and may reduce the risk of cardiovascular diseases (CVD). ${ }^{16}$ The evidence appears to be the strongest for reduced mortality from coronary heart disease (CHD) and stroke. ${ }^{17}$ For instance, 3 large scale studies, the Gruppo-Italiano per lo Studio della Sopravvivenza (GISSI)-Prevenzione Trial $^{18,19}$, the Dietary and Reinfarction Trial (DART) ${ }^{20}$, and the Japan EPA Lipid Intervention 
Study (JELIS) ${ }^{21}$, have all shown that EPA and DHA from oily fish and EPA alone significantly reduce the risk of cardiac death without affecting the risk of nonfatal coronary events (i.e. myocardial infarction, unstable angina). Because of these and other notable findings, the American Heart Association currently recommends that patients with known CHD consume at least $1 \mathrm{~g} / \mathrm{d}$ of EPA and DHA, while individuals without known CHD consume at least 2 servings per week (500 mg/d EPA + DHA) of oily fish to reduce the risk of cardiovascular diseases. ${ }^{13,22}$

Initial efforts to understand the mechanisms by which omega-3 fatty acids reduce risk of death from CHD focused on their modulation of classic, modifiable CHD risk factors (e.g. high blood cholesterol and triglyceride levels, high blood pressure, overweight and obesity, diabetes and prediabetes, smoking, physical inactivity, unhealthy diet, and stress). ${ }^{23,24}$ However, lowering of serum triglycerides and cholesterol and blood pressure did not explain the observed reductions in risk of sudden cardiac death. ${ }^{24}$ Experimental data from animal and cellular studies investigating mechanisms behind which omega-3 fatty acids exhibit their protective effects suggest that the main effect of these fatty acids are mediated from antiarrhythmic properties. ${ }^{15,24}$

\section{Antiarrhythmic Effects}

Studies in rats and marmoset monkeys performed by Pepe and Mclennan ${ }^{25}$ and McLennan et al. ${ }^{26}$ have shown that long-chain omega-3 fatty acids provide a direct, protective effect on the heart itself. For instance, marmoset monkeys who were fed diets containing tuna fish oil, compared to sunflower oil, showed a reduction in inducible cardiac arrhythmias and also an increased omega-3 fatty acid level in the myocardial membrane (12.6\%-31.3\%, $p<0.0001)$, indicating incorporation of omega-3 PUFA into heart tissue. ${ }^{26}$ Similarly, in dog models of ventricular tachyarrhythmia, infused omega-3 fatty acids (100 mg EPA/kg BW/d for 8 weeks) 
decreased the incidence and severity of arrhythmias during coronary artery occlusion, while animals in the control group developed ventricular tachycardia or ventricular fibrillation after occlusion. ${ }^{27}$ The EPA group also showed a significant increase in omega-3/omega-6 ratio, in myocardial and platelet cell membranes $(p<0.01) .{ }^{27}$ By incorporating more omega-3 fatty acids into cardiac membranes, reduction of the omega-6/omega-3 fatty acid ratio can be achieved, thus shifting the myocardium from a proarrhythmic state to an antiarrhythmic state. ${ }^{28}$ However, the exact mechanisms of antiarrhythmic effects of omega-3 PUFA have yet to be clearly established. It has been proposed that these specific mechanisms have a direct stabilizing effect of omega-3 fatty acids on the myocardium itself. ${ }^{13}$ There are many proposed mechanisms of antiarrhythmic effects of omega-3 PUFA ${ }^{29}$, but perhaps the most extensively studied mechanisms are the modulation of ion channels and modification of heart rate variability.

\section{$\underline{\text { Ion Channels }}$}

Antiarrhythmic effects may be mediated from ion channels that underlie the excitation of myocytes in the ventricular myocardium. ${ }^{22}$ The presence of omega-3 fatty acids in cardiomyocyte membrane phospholipids has been found to reduce electrical excitability and modulate the activity of specific ion channels (e.g. sodium, potassium, calcium) in the sarcolemma, despite low concentrations. ${ }^{15,}{ }^{30}$ Investigations using isolated cardiac myocytes in neonatal rats revealed that when arrhythmogenic toxins were added to the myocyte perfusate to induce tachycardia, contracture, and fibrillation, the addition of EPA before toxins were added prevented tachyarrhythmia, and after toxins were added immediately terminated arrhythmias. ${ }^{31}$ These antiarrhythmic actions occurred with both omega- 6 and omega-3 PUFA, specifically, LA, AA, ALA, EPA, and DHA. However, LA and AA were considered inconsistent because in one-third of the tests they either had no antiarrhythmic action or induced violent 
arrhythmias due to cyclooxygenase metabolites of AA. ${ }^{31}$ When cyclooxygenase inhibitors were added with AA to the perfusate, antiarrhythmic effects were similar to those of EPA and DHA. ${ }^{31}$

Actions of omega-3 fatty acids change the electrophysiology of the myocyte by hyperpolarizing the resting membrane potential and increasing the electrical threshold for opening of the fast-acting sodium ion channel. ${ }^{31,32}$ Both effects require an increase in the electrical stimulus to elicit an action potential and prolong the refractory period of the myocyte. $^{30,31}$ This is thought to occur from omega-3 fatty acids ability to inhibit the conductance of voltage-dependent sodium ion channels in the myocyte sarcolemma. ${ }^{30-32}$ It also occurs from inhibition of L-type calcium ion channels in which arrhythmias are induced by excessive cytosolic $\mathrm{Ca}^{2+}$ fluctuations that result in delayed after-potentials, thus triggering a fatal cardiac arrhythmia. ${ }^{30,31,33}$ The ability of omega-3 PUFA to inhibit the L-type calcium ion current prevents excessive cytosolic $\mathrm{Ca}^{2+}$ fluctuations and the likelihood of an arrhythmia to occur. ${ }^{31}$, ${ }^{33}$ Overall, it has been claimed that the combined effects on these 2 ion channels by long-chain omega-3 fatty acids promotes electrical stability in the cell and thereby prevents cardiac arrhythmias. ${ }^{15,30-33}$

\section{Heart Rate Variability}

In addition to their antiarrhythmic effects on ion channels, long-chain omega-3 fatty acids can influence heart rate variability that may exhibit antiarrhythmic effects through the autonomic nervous system. More importantly, reduced heart rate variability is a strong predictor of cardiac arrhythmias and sudden cardiac death, especially in patients with a previous myocardial infarction (MI). ${ }^{14,22}$ A high intake of omega-3 PUFA has been associated with an increased heart rate variability in survivors of $\mathrm{MI}$ and in healthy men. ${ }^{34,35}$ Christensen et al. ${ }^{34}$ demonstrated that 
improved heart rate variability is positively correlated with the content of omega-3 PUFA in cell membranes (primarily DHA) of post-MI patients who consumed one fatty fish meal per week. The same group also reported similar findings in healthy male subjects who received supplementation of 2.0 or $6.6 \mathrm{~g} / \mathrm{d}$ omega-3 PUFA for a 12 -week period, which resulted in an increased heart rate variability. ${ }^{35}$ However, a beneficial effect on heart rate variability was only seen in men with low heart rate variability before dietary supplementation. This was due to the increase of DHA concentration in cell membranes, revealing a dose-dependent effect of omega-3 PUFA on heart rate variability. ${ }^{35}$ Results from both studies indicated that the effects of omega-3 PUFA on heart rate variability are indeed antiarrhythmic and suggest that regular intake of omega-3 fatty acids, whether from fish consumption or supplementation, do have a protective effect against sudden cardiac death (SCD). ${ }^{34,35}$ Both studies also demonstrated that DHA might potentially be the principal omega-3 fatty acid that provides protection against cardiac arrhythmias. $^{34,35}$

\section{Prevention of Sudden Cardiac Death}

Clearly, evidence from experimental trials demonstrates that omega-3 fatty acids may reduce the risk of CHD mortality and sudden cardiac death (SCD). This is of particular interest because ventricular arrhythmias are implicated in $80-90 \%$ of SCD. ${ }^{22}$ It has been shown that victims of SCD possess a lower content of omega-3 PUFA in their coronary arteries compared to controls, which is consistent with evidence on antiarrhythmic mechanisms of omega-3 fatty acids shown in various animal and in vitro studies. ${ }^{30-34,36} \mathrm{~A}$ review of primary and secondary prevention trials evaluating effects of dietary intake and total mortality conducted by Mozaffarian ${ }^{37}$ revealed that all of these studies concluded that moderate consumption of fish or fish oil (1-2 servings/week of fatty fish or 250-500 mg EPA+DHA) substantially reduces the risk 
of CHD death and SCD. A pooled analysis reported that modest consumption compared to no consumption results in a 36\% lower risk of CHD death with little additional benefit associated with higher intakes. ${ }^{37}$ The strength, consistency, and magnitude of the dose-response in each investigation are undoubtedly significant.

\section{Ventricular Arrhythmias}

The prominent reduction in SCD risk further contributes to the vast amount of evidence that omega-3 fatty acids are indeed antiarrhythmic and may prevent fatal ventricular arrhythmias in high-risk patients. Because SCD is often the result of a ventricular arrhythmia, implantable cardio-defibrillators (ICD) are commonly being used in patients who have already experienced a life-threatening ventricular tachycardia/ventricular fibrillation (VT/VF) event. ${ }^{22}$ This prompted several investigations to evaluate the beneficial effects of omega-3 fatty acids for secondary prevention in patients with high-susceptibility to recurrent cardiac arrhythmias.

A randomized control trail conducted by Raitt et al. ${ }^{38}$ consisted of 200 patients with ICDs due to recent episodes of VT/VF events. Patients were randomly assigned to receive $1.8 \mathrm{~g} / \mathrm{d}$ of fish oil (72\% n-3 from EPA/DHA) or a placebo (olive oil) and were followed up for 2 years with monthly clinic visits for the first 3 months and every 3 months thereafter. ${ }^{38} \mathrm{RBC}$ membrane levels of omega-3 fatty acids in patients assigned to the fish oil increased significantly from $4.7 \%$ at baseline to $6.8 \%$ at 1 month and continued to increase to $8.3 \%$ at 3 months, with no significant change after that $(p<0.01) .{ }^{38}$ Patients assigned to the placebo group had no significant change in RBC omega-3 fatty acid levels during the 24-month period. Unexpectedly, patients assigned to the fish oil group had greater incidence of ICD therapy for VT/VF compared to the placebo group at 6, 12, and 24 months after randomization (46\%, 51\%, 65\% for fish oil and 36\%, 41\%, $59 \%$ for placebo, respectively). ${ }^{38}$ Recurrent episodes of VT/VF were more common in patients 
randomized to receive fish oil supplementation $(p<0.01) .{ }^{38}$ The discovery in this study that fish oil did not reduce the risk of VT/VF suggests that omega-3 PUFA may be proarrhythmic in this particular population and is contrary to the evidence suggesting antiarrhythmic properties of omega-3 fatty acids.

On the other hand, the Fatty Acid Anti-Arrhythmia Trial (FAAT) ${ }^{39}$ included 400 patients with an ICD who had either a history of sustained VT/VF or syncope with sustained VT/VF in the past 12 months. Patients were randomized to either $4 \mathrm{~g}$ fish oil (2.6 g EPA/DHA) or $4 \mathrm{~g}$ olive oil capsules daily for a 12-month period. The fish oil supplementation group had a trend toward a prolonged time to the first ICD event or death/adverse event from any cause (risk reduction $=28 \%, p=0.057$ ) compared to the placebo group. ${ }^{39}$ After inclusion of therapies for possible episodes of VT/VF, the risk reduction became significant at 31\% $(p=0.033){ }^{39}$ However, noncompliance was high among patients (35\%) so a separate analysis was conducted on patients who stayed within compliance for 11 months, which yielded a greater risk reduction of $38 \%(p=0.034) .{ }^{39}$ The antiarrhythmic benefits seen in this trial suggests that omega-3 fatty acids may be an alternative to antiarrhythmic drugs for reducing recurrent VT/VF episodes in patients with ICD, thereby preventing risk of fatal cardiac arrhythmias in those at high risk. ${ }^{39}$

The Study on Omega-3 Fatty Acids and Ventricular Arrhythmia (SOFA) ${ }^{40}$ had a total of 546 patients with ICD who had a sustained VT/VF episode within the last 12 months and randomly received either a 2 g/d fish oil (900 mg EPA/DHA) or 2 g/d placebo (high oleic acid sunflower oil) for approximately one year. After follow-up, 75 patients (27\%) of the fish oil group and 81 patients (30\%) in the placebo group experienced an appropriate ICD intervention for VT/VF episodes. ${ }^{40}$ In a subgroup analysis of patients who previously had a MI before study start date $(n=342)$ there was a tendency toward a longer event-free in the fish oil group 
compared with the placebo $(p=0.13) .{ }^{40}$ Of this subgroup 47 patients $(28 \%)$ in the fish oil group reached the primary endpoint (ICD intervention) compared to 61 patients (35\%) in the placebo group, indicating a slight beneficial effect. ${ }^{40}$

Overall, the Raiit ${ }^{38}$ and SOFA ${ }^{40}$ trials did not find strong evidence of a protective effect of omega-3 PUFA intake from fish oil supplements against ventricular arrhythmias in patients with ICDs. Although the Raiit ${ }^{38}$ trial raised the possibility that fish oil may be proarrhythmic, the SOFA $^{40}$ trial did not discover any proarrhythmic properties of fish oil supplements in high-risk patients. The inconsistency between results of these 3 studies suggests that omega-3 fish oil supplementation may or may not possess antiarrhythmic properties in different patient populations. The 2 trials $\left(\mathrm{FAAT}^{39}\right.$ and $\mathrm{SOFA}^{40}$ ) that found greater protective effects of fish oil in post-MI patients against arrhythmia are consistent with findings in other studies ${ }^{18-20}$ and suggest that the benefit of fish oil might be confined to patients with prior MI. Data from these trials can also be interpreted as evidence that habitual intake of fish oil supplements in patients with ICDs and recurrent ventricular arrhythmias should not be recommended. ${ }^{38}$

Intermediate Chain Omega-3 Fatty Acids and Ventricular Arrhythmias

A large amount of research has been conducted on long-chain omega-3 fatty acids, while supporting evidence on intermediate chain omega-3 fatty acids and cardiac arrhythmia risk is less established. ${ }^{16,22} \mathrm{~A}$ direct benefit of antiarrhythmic effects of intermediate chain omega-3 fatty acids, particularly ALA, is largely from epidemiological data. ${ }^{16}$ For instance, only 2 largescale, observational studies have specifically examined the association between ALA intake and SCD. In the Health Professional Follow-up Study, consisting of 45,722 men, ALA intake was not significantly associated with risk of SCD, but a relationship was seen when long-chain 
omega-3 PUFA (i.e. EPA/DHA) intakes were low $(<100 \mathrm{mg} / \mathrm{d}) .{ }^{41}$ This suggests that intermediate chain omega-3 fatty acids from plant sources may decrease SCD risk in men when intake of long-chain omega-3 fatty acids are low, which may potentially have significant relevance in populations with low consumption or availability of fatty fish. ${ }^{41}$ However, it was concluded that the antiarrhythmic effects of EPA and DHA might predominate due to their association with a 40-50\% lower risk of SCD. ${ }^{41}$

On the other hand, in the Nurses' Health Study, ALA intake was inversely associated with risk of SCD among 76,763 women even after controlling for other dietary fats including long-chain omega-3 fatty acids. ${ }^{42}$ Women in the highest 2 quintiles of ALA intake had a 38-40\% lower SCD risk than compared to those in the lowest quintile. This inverse association suggests that ALA may influence cardiovascular risk through effects on arrhythmogenesis and fatal ventricular arrhythmias that may be due to the conversion of ALA to EPA and DHA (although limited) because both have known anti-arrhythmic effects. ${ }^{42}$ The contradictory findings of these particular studies raise the possibility that associations between intermediate-chain omega-3 fatty acids and cardiac arrhythmias/SCD may differ between men and women, but further research is needed.

Although some animal studies have shown potential antiarrhythmic effects of plantbased omega-3 fatty acids ${ }^{43-45}$, clinical trials have yet to support this evidence in humans. ${ }^{16} \mathrm{Few}$ randomized, dietary intervention trials have been able to reach firm conclusions on ALA intake in regards to sudden cardiac death and/or arrhythmias. The Lyon Diet Heart Study ${ }^{46}$ and IndoMediterranean Diet Heart Study ${ }^{47}$ reported significant reductions in SCD in patients consuming an ALA-rich diet (50-70\%, $p=0.015$, respectively), but these studies have several limitations such as uncertainty of whether high intake of ALA is solely responsible for SCD reductions, 
inability to precisely determine ALA intake, flaws in methodology, etc. ${ }^{46,47}$ Randomized trails regarding ALA supplementation in patients have also yet to provide consistent and conclusive results. ${ }^{16}$

\section{Coronary Artery Bypass Graft}

Coronary artery bypass grafts (CABG) are commonly used as a treatment alternative for CAD management when previous therapies have failed. However, there is little evidence regarding omega-3 PUFA supplementation as a secondary prevention therapy after surgical intervention. This is a contrast to the wealth of literature suggesting benefits from omega-3 PUFA supplementation in individuals with known CAD. ${ }^{48} \mathrm{~A}$ large-scale $(\mathrm{n}=610)$, randomized controlled trial called the Shunt Occlusion Trail (SOT) ${ }^{49}$ was one of the first efforts to investigate the effect of omega-3 PUFA supplementation in patients undergoing CABG. Patients were assigned to receive either $4 \mathrm{~g}$ of fish oil or to the control group, while continuing antithrombotic medications (aspirin or warfarin). The primary endpoint of the study was one-year graft patency, which is the likelihood that the graft remains open and free of significant stenosis and/or occlusion. ${ }^{49}$ It was found that omega-3 PUFA supplementation significantly reduced the occurrence of vein graft occlusion. An inverse relationship between serum phospholipid omega-3 fatty acids and vein graft occlusion was also observed. ${ }^{49}$

In addition, a recent cohort study conducted by Benedetto et al.. ${ }^{48}$ investigated the impact of omega-3 PUFA supplementation after CABG. The researchers discovered that omega-3 PUFA supplementation was independently associated with a reduced risk of repeat revascularization and overall mortality in patients with poor left ventricular function. ${ }^{48}$ Of interest, the significant reduction in those with poor ventricular function supports the 
antiarrhythmic actions of omega-3 PUFA. ${ }^{48}$ The general tendency exhibited toward reduced rates of recurring revascularization in those treated with omega-3 fatty acids proposed that omega-3 PUFA may have a protective role in graft and native coronary vessels. ${ }^{48}$ Above all, results from both of these studies strongly support the use of omega-3 PUFA supplementation as a cardiovascular medical therapy in patients undergoing and/or after CABG. ${ }^{48,49}$

\section{Anti-Inflammatory Effects and Atherosclerotic Plaque Progression and Vulnerability}

It has recently been acknowledged that the well-documented anti-inflammatory effects of omega-3 fatty acids might also play a role in acute cardiovascular events. ${ }^{15}$ Inflammation is known to contribute to the development and progression of atherosclerosis. Therefore, the antiinflammatory effects of omega-3 fatty acids could help modify progression of the disease by decreasing the inflammatory activity and environment associated with atherosclerosis. ${ }^{15}$ The atherosclerotic process begins when plaque develops in the vascular wall and with time accrues and produces ischemic conditions from insufficient blood flow or by rupturing, forming a thrombus and occluding the lumen of the vessel. ${ }^{1}$ Atherosclerotic plaque can be described as vulnerable or unstable, suggesting a high risk of rupture, which leads to thrombus formation, thereby increasing risk for myocardial infarction (MI) and stroke. ${ }^{1}$ Vulnerable plaque is characterized by lesions with a thin, fibrous cap, few smooth muscle cells, increased amount of macrophages, and a large lipid core that is composed of cholesterol, lipids, and dead foam cell remnants. $^{1,2}$

When atherosclerotic plaque ruptures, the plaque is exposed to a highly prothrombotic environment of the vessel lumen, triggering an acute inflammatory event. The instability of plaque is dependent upon the thinning of its fibrous cap, enlargement of the lipid core, and high inflammatory content from macrophage infiltration ${ }^{2-4}$, all of which increase the risk of 
intraplaque hemorrhage and rupture. ${ }^{3,4}$ When intraplaque hemorrhage occurs, red blood cells (RBC) enter the atherosclerotic lipid core of the plaque, resulting in an increase of cholesterol accumulation due to the large amounts of free cholesterol contained in RBC membranes. ${ }^{2-4} \mathrm{RBC}$ membranes are 1.5-2.0 times richer in cholesterol than any other cell in the human body and approximately $40 \%$ of its cell weight is composed of lipids. ${ }^{2}$ Because RBCs cannot synthesize lipids de novo nor esterify cholesterol, the majority of lipids found within the membrane originate from an exchange with plasma lipoproteins. ${ }^{50}$ Tziakas et al.. ${ }^{2}$ and references therein, indicated that there are several mechanisms responsible for the transfer of plasma cholesterol to RBCs such as the free cholesterol content in lipoprotein particles, number and distance of transfer sites on RBC membranes, structure and composition of RBC membrane, especially sphingomyelin content due to its molecular attraction for cholesterol and its proposed ability to "trap" unesterfied cholesterol within a particular membrane site. With that being said, the cholesterol concentration in RBC membranes must reflect an individual's lipid profile over a long period of time. Therefore, it is plausible that the cholesterol content of RBC membranes is an index of plasma cholesterol concentrations indicative of the lipids consumed over the last several months. ${ }^{2}$ The RBC with its lipid-rich membrane can contribute to the cholesterol found in atherosclerotic plaque due to the high cholesterol content exceeding that of all other cells. ${ }^{2}$ Thus, it can be postulated that most of the free cholesterol found in atherosclerotic plaque is derived from RBC membranes and can lead to plaque instability as seen in patients with CAD. ${ }^{2}$

It has been well established that long-chain omega-3 PUFA can decrease postprandial and fasting plasma triglyceride concentrations. ${ }^{15}$ It has also been shown that long-chain omega-3 fatty acids from dietary fish oil reduces atherosclerosis in rhesus monkeys ${ }^{51}$, pigs ${ }^{52}$, and rabbits ${ }^{53}$ due to combination of lipid-lowering effects and decreased inflammation. ${ }^{10,15}$ As previously 
mentioned, omega-3 PUFA have showed significant results on reduction of SCD ${ }^{22,37}$ even in the absence of lipid reduction. ${ }^{19}$ On the other hand, omega-6 PUFA, particularly linoleic acid, has been demonstrated to promote inflammation that could contribute to plaque instability with excessive intake. ${ }^{54}$

\section{PUFA Incorporation into Atherosclerotic Plaque}

If PUFA are to affect plaque instability then they must be incorporated into the lipid membranes and other components of the atherosclerotic lesion itself. A study by Rapp and colleagues ${ }^{55}$ demonstrated for the first time that dietary omega-3 fatty acids are rapidly incorporated into the lipid classes of advanced atherosclerotic lesions. Study participants had obstructive atherosclerosis and consumed fish oil for 1-16 weeks (6-120 days) before scheduled peripheral vascular surgery. It was discovered that concentrations of omega-3 PUFA (EPA and DHA) in plaques removed at surgery were much higher than those removed in plaques from the control group..$^{55}$ EPA and DHA accounted for more than $80 \%$ of the omega-3 fatty acid accumulation in the cholesterol ester fraction of plaque. The total mean of omega-3 PUFA in the cholesterol ester was $4.9 \pm 3.1 \%$ in plaques of the fish-oil fed group, a 350\% difference compared to controls $(p<0.001) .{ }^{55}$ Incorporation of DHA and EPA appeared to be linear over time, unlike plasma in which the initial increase was found to be exponential until it reached a steady state around 3 weeks of supplementation. ${ }^{55}$ It was also discovered that the accumulation of omega-3 fatty acids in phospholipids increased with the length of the feeding period, resulting in $8.8 \%$ of total fatty acids incorporated into phospholipids, compared to $1.8 \%$ for controls (490\% difference) ${ }^{55}$ Total saturated fatty acids reduced from $17.2 \%$ to $13 \%$ in the plaques of fish-oil fed patients $(p<0.005)$. Plasma concentrations revealed a significant decrease in omega-6 PUFA, primarily linoleic acid, in both cholesterol esters and phospholipid fractions $(p<0.001)$ 
with a simultaneous increase in omega-3 fatty acids. ${ }^{55}$ In general, these initial data suggest that atherosclerotic plaque steadily accumulated omega-3 fatty acids from plasma became more polyunsaturated after fish oil supplementation. It was evident that EPA and DHA replaced saturated fatty acids within the cholesterol ester and triglyceride fractions and replaced MUFA in the phospholipid fraction of the lipid bilayer within the lesions. ${ }^{55}$ As a result, the unsaturation index of the lipid classes within the plaques greatly increased, which may possibly affect membrane fluidity ${ }^{55}$ Because lipid composition of the atherosclerotic lesion determines the characteristic state of the plaque (liquid-crystalline, crystalline, oil-droplet) ${ }^{56}$, it may be plausible that omega-3 PUFA supplementation can alter the plaque's physical state and increase fluidity of plaque cellular components and infiltrating lipoproteins from plasma, ${ }^{55,56}$ all of which further contribute to nonfatal and fatal cardiovascular event reduction as a result of enhanced plaque stability associated with increased intake of omega-3 PUFA.

Although these are indeed novel findings, several limitations exist within the Rapp investigation. Most notably, there were inconsistency with types of atherosclerotic plaques (carotid, aortic, iliac, femoral) within the patient group and patients were receiving a very high dose of fish oil (16-21 g/d EPA \& DHA). A dosage this high compared to the average consumption of EPA and DHA in Western diets $(<0.3 \mathrm{~g} / \mathrm{d}){ }^{12}$ can be considered unrealistic for habitual intake. Also by comparison, secondary prevention trials ${ }^{18-21,47,57}$ that demonstrate cardio-protective effects provide $\leq 1.8 \mathrm{~g} / \mathrm{d}$ EPA $\&$ DHA, an amount considerably less than the dosage used in the Rapp investigation. This suggests that an excessive dosage of fish oil is not needed to obtain cardiovascular protection. In addition, these substantial limitations associated with the Rapp investigation prompted Thies and colleagues ${ }^{58}$ to investigate whether moderate consumption of omega-3 PUFA would result in DHA/ EPA incorporation into atherosclerotic 
plaque lipids resulting in improved plaque stability and if intake of sunflower oil would result in omega-6 PUFA incorporation into atherosclerotic plaque lipids resulting in reduced plaque stability. Plaque morphology and degree of macrophage infiltration determined the patients' plaque stability of carotid arteries. Patients were randomized to receive control oil (80:20 blend of palm and soybean oil), sunflower oil (3.6 g/d), or fish oil (1.4 g/d) for a mean duration of 42 days in all groups. ${ }^{58}$ Over the period studied, researchers concluded that sunflower oil supplementation (40\% increase in daily consumption) did not result in increased incorporation into carotid plaques, therefore not leading to altered plaque stability. ${ }^{58}$

On the other hand, it was also observed that at a modest dose of supplementation, longchain omega-3 PUFA are readily incorporated into atherosclerotic plaque lipids, while simultaneously lowering plasma triglyceride concentrations. ${ }^{58} \mathrm{EPA}$ incorporation into plaque lipids, particularly phospholipids, was noted to be linear with time. ${ }^{58}$ This indicates that even at a modest level, incorporation of omega-3 PUFA can happen within a short time, suggesting that stability can be enhanced in atherosclerotic plaques even at an advanced stage of atherosclerosis. ${ }^{58}$ Plaque morphology measures also indicated that the fish-oil group had more stable plaque, characterized by a well-formed fibrous cap, rather than thin inflamed caps as seen in the other 2 groups. ${ }^{58}$ Thies et al. ${ }^{58}$ attributed the differences between groups to higher EPA and DHA concentration in plaque lipids, indicating that these particular fatty acids play a key role in establishing plaque vulnerability. Plaques from the fish oil group were less infiltrated with macrophages, suggesting that the primary effect of fish oil on plaque inflammation and instability might involve modulation of macrophage aggregation and function. ${ }^{58}$ 


\section{Clinical Implications}

Findings from both Rapp ${ }^{55}$ and Thies ${ }^{58}$ propose significant clinical relevance in progression of the atherosclerotic disease process. Both studies indicate that plaque stability is the primary determinant of rupture and thrombosis-mediated acute coronary events rather than the exact degree of atherosclerosis. ${ }^{55,58}$ Acute coronary events, also known as acute coronary syndromes (ACS), have not only been attributed to the lumen narrowing from disease progression but also from the rupture or erosion of atherosclerotic plaques with superimposed thrombus formation. ${ }^{2}$ Plaque rupture with superimposed thrombosis accounts for $60-75 \%$ of all ACS (unstable angina, myocardial infarction). ${ }^{2}$ The stabilization of carotid plaques through omega-3 PUFA supplementation can decrease ACS and risk of neurological events (e.g. stroke, TIA, cerebral aneurysm) in those with advanced atherosclerosis. ${ }^{55,58}$ Likewise, fewer macrophages in carotid plaques can also decrease the frequency of neurological events. ${ }^{58,59}$ Therefore, it is logical to postulate that the effects of fish oil can inhibit the development of plaque instability in both advanced disease and early atherosclerosis. ${ }^{58}$ These effects could also apply to lesions throughout the vasculature including coronary arteries ${ }^{58}$, further elucidating the mechanisms behind which fish oil exhibits such a strong, protective effect against fatal MI. ${ }^{18-21,47,57}$

Moreover, this proposes additional clinical significance of the mechanisms behind which RBC membranes contribute to the progression of atherosclerosis and plaque instability. Cholesterol accumulation during atherosclerotic lesion progression is a principal contributing factor in plaque vulnerability. ${ }^{5,60}$ The substantial amount of cholesterol within RBC membranes has been recognized to contribute to the amount of free cholesterol within the necrotic lipid core of atherosclerotic lesions. ${ }^{2,61}$ It has been demonstrated that during plaque progression of an 
atherosclerotic lesion, (fatty streaks to intermediate plaque to advanced plaque) free cholesterol concentration significantly increases over time. ${ }^{62}$

The source of RBCs within coronary lesions is provided by leaky immature blood vessels that surround the plaque. ${ }^{50}$ After an intraplaque hemorrhage occurs, RBCs rapidly enter the plaque, accumulating free cholesterol and expanding the necrotic core. This increase in free cholesterol is associated with lesion instability. ${ }^{5,}$ Moreover, the hemorrhaging within the plaque also leads to excessive macrophage infiltration, which further contributes to the instability of the plaque via interactions between free cholesterol and macrophages in the necrotic core., 50 Increased free cholesterol content in macrophages may promote macrophage apoptosis and lead to increased foam cell production, a key event in the destabilization of atherosclerotic plaque. $^{2}$ The release of RBCs into the lesion from the thinned, fibrous cap also contributes to plaque instability by promoting an inflammatory cascade mediated by a variety of mechanisms, including hemoglobin toxicity, cholesterol crystallization, and cytokine release from RBC membrane receptors. ${ }^{2}$ Recent studies associating ACS with increased RBC membrane cholesterol and interleukin-8 content support the role of RBC in plaque vulnerability and atherosclerosis progression. ${ }^{2}$ Inverse relationships have also been shown between EPA content in plaque phospholipids and plaque inflammation and instability, suggesting that the more EPA there is within the plaque, the more stable it is ${ }^{63}$ Supplementation of omega-3 PUFA ethyl esters has been shown to result in fewer foam cells within the plaque and lower T-cell content related to increased content of EPA. Such observations indicate that EPA may exclusively play a key role in reducing inflammation and stabilizing atherosclerotic plaque.$^{63}$ In general, the higher the oxidative stress/inflammation and concentration of cholesterol within the plaque, the more likely an ACS event will occur. 


\section{$\underline{\text { Red Blood Cell Membrane Fatty Acid Composition }}$}

\section{$\underline{\text { Red Blood Cell Fatty Acid Patterns and Acute Coronary Syndrome }}$}

The observation of higher cholesterol content in RBC membranes of patients with ACS compared to those with stable CAD, demonstrated by Tziakas et al. ${ }^{2}$, proposes a significant association between clinical instability and RBC membrane cholesterol content. However, the association between long-chain omega-3 PUFA and risk of nonfatal cardiac events remains controversial. For instance, results from a cohort study consisting of only women ${ }^{64}$ indicated that higher fish intake is significantly associated with reduced risk of MI, while a study of only men ${ }^{65}$ had no relationship between fish intake and MI risk. Although significant reductions in sudden death have been observed through EPA/DHA supplementation ${ }^{19}$ and increased fish consumption $^{20}$, significant impact on MI risk was not observed during these treatments. However, recent evidence has shown that a diminished RBC level of EPA and DHA (\% of total fatty acids) identifies patients at an increased risk for nonfatal CHD events. ${ }^{66}$ A $20 \%$ difference in EPA and DHA content was found between ACS cases and controls and those within the lowest quartile for EPA/DHA content had a 3-fold increase of ACS compared to those with the highest level of EPA/DHA content. ${ }^{66}$ Even after controlling for several ACS risk factors (lipid levels, BMI, alcohol use, DM, HTN, MI, family history of CAD, smoking habit, statin/antiplatelet drug use, education level), this biomarker remained a significant predictor of case status. $^{66}$

A similar study investigated whether RBC fatty acid pattern discrimination between case and control groups would supplement the predictive value of standard CHD risk factors from the widely used Framingham Risk Score (age, sex, total cholesterol, HDL cholesterol, HTN, DM, 
smoking status) ${ }^{67,68}$ Surprisingly, it was discovered that only 2 factors, HDL cholesterol and smoking habit, were significantly related to ACS case status. ${ }^{68}$ It was also found that ten FA were significantly related to ACS case status; however, 2 FA (eicosadienoic acid; trans oleic/elaidic acid) were directly related, while the remaining 8 FA were inversely related with odds for ACS case status. ${ }^{68}$ These remaining FA included both parent omega-6 and omega-3 PUFA and their derivatives (except EPA), the MUFA palmitoleic acid, and the saturated fatty acid, stearic acid. The FA with the greatest impact was linoleic acid and is expected because it is the most abundant EFA in the diet. ${ }^{68}$ These findings indicated that identification of RBC-FA patterns discriminate ACS cases from controls better than standard risk factors, resulting in substantial improvement in prediction of nonfatal cardiac events compared to traditional CHD risk factors (i.e. Framingham Risk Factor). ${ }^{68}$ A recent case-control study from Park et al.$^{69}$ in patients with nonfatal MI supports the greater ability of RBC-FA profiles for ACS risk identification.

\section{Omega-3 Index}

The use of RBC membrane fatty acid (FA) content has become increasingly used as a biomarker because it reflects both dietary intake and metabolism processes ${ }^{68}$ It is becoming more preferred than plasma fatty acids because RBC FA reveal long-term dietary fats consumed and is not affected by recent dietary fat intake. ${ }^{6,7} \mathrm{RBC}$ membranes also reflect the amount of omega-3 PUFA incorporated into cardiac membrane phospholipids, which proposes significant relevance of RBC FA composition because of the beneficial effects of omega-3 PUFA on cellular function arise from their actions on and within cell membranes. ${ }^{70}$ The recently coined "Omega-3 Index" determines the sum of EPA and DHA content in RBC membranes expressed as a percentage of total RBC FA and is significantly correlated with myocardial omega-3 PUFA content. ${ }^{70-72}$ Thus, the index is becoming more widely known as an additional risk factor for 
CHD and SCD. ${ }^{70,71}$ The researchers who developed the omega-3 index have proposed that a concentration of $8 \%$ offers the greatest degree of cardio-protection, while a value of $\leq 4 \%$ is associated with the highest risk for CHD death. ${ }^{70,71}$ These proposed omega-3 index categories have been further supported by Block et al... ${ }^{6}$, which revealed that those in the $\geq 8 \%$ category are about $70 \%$ lower for ACS risk than those in the $\leq 4 \%$ category.

For fatal cardiac events, such as primary cardiac arrest, Siscovick et al.. ${ }^{73}$ determined whether dietary intake and RBC EPA and DHA content were associated with risk of cardiac arrest. It was found that an inverse relationship of both dietary intake and RBC membrane levels of omega-3 PUFA with risk of primary cardiac arrest exist. For instance, the data showed that an intake of $5.5 \mathrm{~g}$ of omega-3 PUFA (one fatty fish meal per week) was associated with a 50\% risk reduction and an RBC omega-3 level of $4.3 \%$ and $5 \%$ of total fatty acids was associated with a $50 \%$ and $70 \%$ risk reduction, respectively. ${ }^{73}$ After multivariate adjustment, it was still shown that modest consumption of long-chain omega-3 PUFA and small increases (1-2\% of total fatty acids) in omega-3 PUFA levels of RBC membrane are significantly associated with reduced risk for primary cardiac arrest. ${ }^{73}$

In the case of SCD, results from the Physician's Health Study ${ }^{65}$ indicated that whole blood long-chain FA levels (i.e. percent of total FA as EPA/DHA/DPA) at baseline were an independent predictor of SCD risk. It was shown that men with levels in the highest quartile (6.87\% average) had an $81 \%$ lower risk of SCD compared to those in the lowest quartile (3.58\% average). ${ }^{65}$ After controlling for other fatty acids and known confounders, the inverse relationship with subsequent risk of SCD remained statistically significant ${ }^{65}$, suggesting that the omega-3 index may be more informative than other established risk factors in the case of evaluating SCD risk in individuals with CHD. ${ }^{70}$ Collectively, findings from Siscovick et al. ${ }^{73}$, 
Block et al. ${ }^{66}$ and Albert et al. ${ }^{65}$ support the use of Omega-3 Index (or biomarkers convertible to it) as an independent CHD risk factor in both nonfatal and fatal cardiac events. The evidence provided by these 3 studies constitutes the strong, predictive value of this biomarker for primary prevention of CHD. Unfortunately, not all studies evaluating the clinical utility of EPA and DHA biomarkers have found such positive results. Aarsetoey et al. ${ }^{74}$ did not confirm a mortality (e.g. all-cause and cardiac) benefit, despite an observed omega-3 index of $>5.27 \%$ in patients with ACS. Therefore, this study was unable to confirm the omega-3 index as a risk marker after an ACS event. Other omega-6 and omega-3 PUFA-based metrics have been proposed as prognostic risk markers of CHD such as the GRACE score ${ }^{75}$ and the Lands' index ${ }^{76}$, but remain beyond the scope of this review.

\section{Factors Influencing Red Blood Cell Fatty Acid Composition}

When evaluating long-chain omega-3 PUFA content of RBC, it is important to consider factors that influence the incorporation of EPA and DHA into RBC membranes. Sands et al. ${ }^{77}$ investigated whether gender, age, body mass index (BMI), ethnicity, smoking status, and presence of diabetes had an effect on RBC EPA and DHA independent of fish intake. All of the factors, except gender, ethnicity, and smoking status proved to be independent predictors of RBC EPA and DHA content. ${ }^{77}$ The average RBC EPA and DHA content in this sample of individuals ( $n=163$ ) was $4.9 \%$, which is close to the high-risk omega-3 index category for cardiac death. ${ }^{77}$ It was found that with every additional 10 years of age, RBC EPA and DHA content increased by 0.5 units. Itomura et al. ${ }^{78}$ and Gudbyarnason ${ }^{79}$ demonstrated similar associations of increased RBC EPA and DHA content with age in humans and animals, respectively. An increase in BMI (3 numerical values) was found to decrease EPA and DHA proportions by 0.3 units, suggesting that higher BMI values result in lower omega-3 PUFA content in RBC membranes. ${ }^{77}$ Presence of 
diabetes was associated with a 1.13-unit decrease in RBC EPA and DHA, while sex was not demonstrated to be a determinant of RBC EPA and DHA. ${ }^{77}$ With regards to dietary intake, it was shown that for every monthly serving of tuna or other fatty, nonfried fish consumed, RBC EPA and DHA content increased by 0.24 units. ${ }^{77}$ This suggests that consuming more long-chain omega-3 PUFA can easily and quickly increase the omega-3 index; thereby, emphasizing the relationship between this biomarker and dietary intake. ${ }^{71,77}$

Another influential factor to consider is physical activity status. Itomura et al. ${ }^{78}$ reported an inverse association with physical activity and RBC EPA and DHA content. Mechanisms behind this association are not precisely understood but may be attributable to catabolism of EPA and DHA for production of energy when physical activity is increased. ${ }^{78}$ Although both Sands et al. ${ }^{77}$ and Itomura et al. ${ }^{78}$ found that smoking was not associated with reduced levels of EPA and DHA in RBC membranes, lower levels of omega-3 PUFA in RBC of smokers have been observed.$^{65}$ Non-Caucasian ethnicity was found to not be of significant predictive value of RBC EPA and DHA content, while Caucasian descent remained a significant predictor. This may be attributable to that Caucasian Americans are less likely to consume fish than other ethnicities. ${ }^{77}$

\section{$\underline{\text { Diagnostic Implications }}$}

Based on the vast amount of literature previously discussed, the use of RBC membrane FA composition appears to be an ideal biomarker for risk of death from CHD. Because RBCs have a rather long lifespan (90-120 days), the fatty acid profile is considered a better indicator of long-term fatty acid intake compared to the intermediate lifespan (3 weeks-3 months) of platelet

or plasma lipids. ${ }^{64,80}$ Typically, plasma or serum samples are more commonly analyzed for assessment of an individual's fatty acid status (e.g. total cholesterol, LDL, HDL, triglyceride) 
because the fatty acid composition of plasma reflects recent dietary fat intake. ${ }^{80}$ Although, RBC and plasma fatty acids are both reliable to use for evaluating cardiac fatty acid status, it is vital to consider which biomarker provides the best quantitative estimate of dietary intake. ${ }^{64,81}$ Sun et al. ${ }^{64}$ reported that correlations between omega-3 PUFA and trans fatty acid content and corresponding dietary intake measured by validated questionnaires are stronger for RBC than for plasma. It has also been proposed that omega-3 PUFA in RBC are more strongly correlated with intake than adipose tissue, in which fatty acids have an estimated half-life of 480 days. $^{64}$ These findings indicate that RBC may be considered a superior alternative to plasma and adipose tissue measurements when evaluating the relationship of dietary intake and RBC FA composition. Fortunately, Harris and von Schacky ${ }^{71}$ have summarized the potential advantages of RBC membranes as biomarkers for omega-3 PUFA intake. ${ }^{70,71}$ Clinical information obtained from measuring RBC FA composition can not only impact CHD prevention but also the prevention and treatment of inflammatory diseases, degenerative diseases, autoimmune disorders, and other medical conditions that could benefit from assessing fatty acid balance/imbalances. ${ }^{7}$

\section{Food Frequency Questionnaire: Validity and Reliability}

In addition to a direct measurement of fatty acid intake from blood or tissue specimens, an indirect measure such as food-frequency questionnaires (FFQ) and diet records, should be used to help relate and confirm reported findings. Currently, there are several biomarkervalidated PUFA FFQ measures, all of which consists of more than 100 questionnaire items. This prompted Sublette and colleagues to develop a brief dietary assessment of omega-3 PUFAcontaining foods because the majority of omega-3 PUFA comes from a limited array of food

sources. ${ }^{82}$ Based on the National Cancer Institute’s Diet History Questionnaire, Sublette et al. ${ }^{82}$ developed a 21-item questionnaire that included items assessing common types of fish and 
seafood consumed, as well as walnuts, flaxseeds, flaxseed oil, cod liver oil, and canola oil over the past 6 months. The FFQ also included items addressing specific types and dosage of omega-3 PUFA supplements. ${ }^{82}$ Frequency of intake for both food and dietary supplements is assessed on each item with ranges of never to number of times each month, week, and day. An algorithm was also developed to determine individual intakes of long-chain omega-3 fatty acids (ALA, EPA, DHA) to account for portion size, consumption frequency, sex and average omega-3 PUFA content of food choices. ${ }^{82}$

While determining validity of the questionnaire, it was discovered that FFQ responses of EPA/DHA intake were significantly correlated with EPA/DHA plasma levels of study participants. ${ }^{82}$ For example, reported nonintake of DHA appeared to be an accurate indicator of low plasma DHA levels. ALA intake estimated from the FFQ, however, was not correlated with plasma ALA levels, indicating that this questionnaire may not adequately predict ALA intake. Sublette et al. ${ }^{82}$ indicated that this particular finding remains consistent with other reports of no to moderate correlations of FFQ-estimated ALA intake with plasma and RBC levels. ${ }^{82}$ This suggests that both medium and long-term biomarkers (i.e. plasma and RBC, respectively) may not be beneficial in validating dietary intake of ALA.

The significant association of estimated DHA and EPA intake from FFQ items with a plasma biomarker supports the validity of this tool in assessing long-chain omega-3 PUFA intake. ${ }^{82}$ Because EPA and DHA content of RBC has been highly correlated with plasma measures of EPA and DHA, this particular FFQ can also be used with RBC biomarkers. ${ }^{70}$ The brief and inexpensive nature of this instrument supports its beneficial use in clinical settings. On the other hand, several limitations exist with self-reported measures including bias, subjectivity, education level, dependence upon accuracy of memories, and willingness to report details of 
diet. $^{64}$ These effects must be considered when implementing FFQ results for correlation with biomarkers of fatty acid intake.

\section{$\underline{\text { FRAP Assay Implications }}$}

An additional screening tool that may propose clinical significance in individuals with CHD is the measurement of total antioxidant, or reducing, power of plasma. The test, developed by Benzie and Strain ${ }^{83}$, determines the ferric reducing ability of plasma (FRAP) by using antioxidants as reductants in a redox-linked colorimetric method. Antioxidants within the body serve as a means to minimize oxidative stress damage by inhibiting generation of harmful reactive oxygen species (ROS), destroying potential oxidants, and scavenging free radicals. ${ }^{83}$ The purpose of the FRAP assay is to detect a change in absorption when the ferric form of iron (Fe $3^{+}$) reduces to ferrous form $\left(\mathrm{Fe} 2^{+}\right.$) (emitting a strong blue color; Fe $2^{+} / \mathrm{TPTZ}$ ), representing the reducing power of electron-donating antioxidants within the plasma reaction mixture. ${ }^{83}$

The need for FRAP assays in individuals diagnosed with CHD is supported by several studies reporting significant associations between CVD risk and increased oxidative stress related to antioxidant deficiency, susceptibility to lipoprotein oxidation, and degree of obesity. ${ }^{83}$,

${ }^{84}$ In particular, the rate of lipoprotein oxidation proposes great concern because it results in diminished levels of PUFA (mostly long-chain omega-3 PUFA) from free radicals attacking double bonds within lipid membranes, while also reducing RBC antioxidant content (i.e. Vitamin E, beta-carotene, $\mathrm{CoQ}_{10}$, etc.). ${ }^{84}$ Therefore, a decrease in both the degree of unsaturation and antioxidant levels of RBC membranes is anticipated. Additional damaging effects on the RBC membrane include decreased membrane fluidity, decreased membrane-bound enzyme activity, tissue hypoxia, and alterations in membrane structure. ${ }^{84}$ It has also been reported that high cholesterol content of RBC membranes in overweight and obese individuals may also contribute 
to the increased rate of lipid oxidation. ${ }^{84}$ For instance, cholesterol increases within RBC, platelets, and leukocytes have been shown to cause enhanced production of oxygen-free radicals. Because RBCs contain a high amount of oxygen, it provides a convenient environment for cholesterol to exert its pro-oxidant reactions.$^{84}$ Therefore, it is of interest to investigate the level of antioxidant defense and oxidative stress to evaluate whether FRAP assay has the clinical utility to assist in prevention and treatment of CHD, as well as obesity.

\section{$\underline{\text { Conclusion }}$}

It is evident that omega-6 and omega-3 PUFA play a vital role in cardiovascular health. Deficiencies and imbalances of these essential nutrients as well as PUFA composition within cell membranes pose a significant risk for development of CVD. Evidence strongly suggests that increasing intake of long-chain omega-3 PUFA reduces the risk of cardiovascular mortality. Mechanisms through which omega-3 PUFA reduce cardiac mortality include modulation of eicosanoids $^{8,10,14,15}$; reductions of arrhythmias ${ }^{25-27,29,38-40,42,43-45}$, ischemia-reperfusion injury ${ }^{43}$, serum triglyceride levels ${ }^{15,58}$, platelet aggregation and thrombosis ${ }^{8,14,15}$; increased heart rate variability $^{34,35}$, and stabilization of atherosclerotic plaque through decreased inflammation ${ }^{2}$ 15,55,58. Incorporation of PUFA into membrane phospholipids is particularly important because it determines the fatty acid composition of that specific membrane. ${ }^{2}$ RBC FA profiles have become a new approach to evaluate dietary intake of PUFA in relation to CHD risk. ${ }^{6}$ Measurements of PUFA in RBC membranes provide a long-term reflection of intake over the past several months because it is not affected by recent fat intake and has a longer lifespan than that of plasma. ${ }^{2}$ Omega-3 PUFA content in RBCs has been highly correlated with dietary intake of EPA and DHA and is a significant and independent predictor of CHD. ${ }^{66,65,70-73,77}$ The prognostic and/or diagnostic value of this modifiable risk marker can lead to new dietary and clinical 
recommendations for CHD prediction and treatment. Combining this biomarker with another measurement of PUFA intake such as a self-reported questionnaire can help clinical investigators focus on particular foods consumed within prior months to further correlate RBC FA composition with dietary intake. An additional tool to enhance clinical applicability of RBC FA composition is the ferric reducing ability of plasma (FRAP) to determine level of oxidative stress and whether antioxidant therapy may be beneficial in CHD disease treatment/prevention. 


\section{CHAPTER 3}

\section{MATERIALS AND METHODS}

\section{$\underline{\text { Subjects }}$}

Selection of Cases and Controls

Subjects were recruited from the East Tennessee State University Heart Clinic, an affiliate of James H Quillen College of Medicine Division of Cardiology. Inclusion criteria for case subjects included cardiac catheterization within past 3 months of study start date (June 2013); signs of obstructive CAD (standard definition: $50 \%$ left main or left main equivalents or $70 \%$ of other coronary arteries) or any past history of CABG or coronary stent placement; and $\geq 30$ years of age. Participants who were also $\geq 30$ years of age, received cardiac catheterization 3 months prior to study start date, but did not show evidence of CAD ( $<10 \%$ stenosis) were selected for the control group. Exclusion criteria for both case and control groups include known history of CAD, CABG, or coronary stent placement and $<30$ years of age. A total of 39 subjects were included in this study through random selection of medical records or selection of patients through scheduled walk-ins. Of the 39 participants, 3 individuals have had previous catheterizations more than 5 years ago that resulted in either negative or mild findings; therefore, these individuals were included in total sample. One participant was excluded due to incomplete information, resulting in a total sample size of 38 participants. The case group consisted of 9 participants with a significant finding of CAD during catheterization. The remaining 29 participants did not have significant findings during catheterization and were included in the control group. All study subjects provided written informed consent for the study procedures. 
The study was approved by the Institutional Review Board of East Tennessee State University's Office of Research and Sponsored Programs (Appendix A).

\section{Food Frequency Questionnaire}

A food frequency questionnaire (FFQ) designed by Sublette et al. ${ }^{82}$ was given to each study participant immediately after enrollment. Permission was received from ME Sublette for use of the survey for inclusion in this study (Appendix B). The FFQ consisted of 21 items assessing the average omega-3 PUFA intake over the past 6 months and took participants approximately 5 minutes to complete. Items in the FFQ included specific questions on omega-3 PUFA-containing foods and omega-3 PUFA supplements. The FFQ survey can be found in Appendix A. Intakes of ALA, DHA, and EPA were individually calculated according to an algorithm taking into account portion size, frequency of consumption, sex, and average omega-3 PUFA content of the participant's food choices. Self-reported measures of age, sex, ethnicity, smoking status, and physical activity level were also obtained from each participant after completion of FFQ. History of CAD, Diabetes Mellitus, plasma lipid profile, height, and weight were obtained from each participant's medical records. From these records, patient's body mass index (BMI) was calculated for each study participant.

\section{$\underline{\text { Laboratory Methods }}$}

\section{Blood Collection}

After enrollment and completion of FFQ, blood samples were collected from participants into a 5-mL EDTA vacutainer. Within 48 hours after withdrawal, the whole blood sample was then transported to the Human Nutrition and Dietetics Research laboratory at the ETSU 
Valleybrook campus. A fasting state of participants at the time of blood collection was not required for the study.

\section{$\underline{\text { Plasma Separation }}$}

Immediately upon arrival to the laboratory, blood samples were centrifuged at $4000 \mathrm{rpm}$ for 10 minutes at room temperature. The resulting supernatant (upper fraction of EDTA tube) is composed of plasma and was drawn off via Pasteur pipette then transferred to a $3 \mathrm{~mL}$ glass amber vial. These vials were stored at $-30^{\circ} \mathrm{C}$ until further analysis. The remaining blood sample (lower fraction) was then reconstituted with equal parts saline solution $(0.9 \% \mathrm{NaCl})$, briefly vortexed to disrupt the RBC pellet, and centrifuged for 5 minutes (@ 4000 rpm). The colorless and clear supernatant layer was removed and discarded and the washing procedure was repeated 3 times.

\section{$\underline{\text { Isolation of Red Blood Cells and Extraction of Lipids }}$}

Lipid extraction and methylation of RBC was accomplished by modifying the simplified procedure developed by Kang and Wang (2005). The final-washed RBC pellet was resuspended in $25-\mu \mathrm{L}$ saline solution $(0.9 \% \mathrm{NaCl})$ and briefly vortexed $(\approx 60 \mathrm{sec})$. One hundred $\mu \mathrm{L}$ of $\mathrm{RBC}$ were extracted and transferred to a clear, $150 \mathrm{~mm}$ x $16 \mathrm{~mm}$ screw-top test tube with phenolic, Teflon-lined screw caps. This step was performed twice to produce duplicate samples. RBC were directly methylated by adding 2 mL of hexane (Optima; BP2615; Fisher Scientific, Fair Lane, NJ, USA) and 2 mL of boron triflouride-methanol solution (B1252; Sigma-Aldrich, St Louis, MO, USA), vortexed until RBC were well mixed, and heated for 1 hour at $100^{\circ} \mathrm{C}$ in a heating block to generate fatty acid methyl esters (FAME) from the RBC membrane phospholipid. The methylated fatty acid solution was then cooled to room temperature. A total of $1.5 \mathrm{~mL}$ of 
deionized distilled water (DDW) was added to the test tube, vortexed for about 60 seconds, and centrifuged at 4000 rpm for 1 minute. The upper hexane layer was extracted, transferred to a new, clear test tube, and dried under nitrogen in a heated water bath $\left(50^{\circ} \mathrm{C}\right)$. After evaporation, FAME were resuspended in $250 \mu \mathrm{L}$ of hexane and transported to a $1.5 \mathrm{~mL}$ amber glass autosampler vial. A low volume insert (300 $\mu \mathrm{L}$; (National; Fisher Scientific, Fair Lane, NJ, USA) was added to each vial to assist in the autoinjection of the sample to the gas chromatograph. Five $\mu \mathrm{L}$ of C-17 internal standard (1:9 hexane dilution; (Nu-Chek Prep, Inc., Elysian, MN, USA) was also added to each insert within the vials and stored at $-30^{\circ} \mathrm{C}$ until further analysis.

\section{Analysis of Fatty Acids}

FAMEs were analyzed by flame ionization gas chromatography (GC) (Shimadzu GC2010; Shimadzu Corporation, Kyoto Japan) using a capillary column (Zebron ZB-WAX, 30 m length, $0.25 \mathrm{~mm}$ i.d., $0.25 \mu \mathrm{m}$ film thickness; Phenomenex, Torrance, CA, USA). Column conditions included the carrier gas (helium) flow rate of $30 \mathrm{~mL} / \mathrm{min}$ and a temperature program of a constant temperature ramp $\left(2^{\circ} \mathrm{C} / \mathrm{min}\right)$ at an initial temperature of $160^{\circ} \mathrm{C}$, held for $5 \mathrm{~min}$; $170^{\circ} \mathrm{C}$ held for 8 minutes; $180^{\circ} \mathrm{C}$ held for 10 minutes; $190^{\circ} \mathrm{C}$ held for 15 minutes; $200^{\circ} \mathrm{C}$ held for 15 minutes; and final oven temperature of $210^{\circ} \mathrm{C}$ held for 20 minutes. Additional instrument conditions included: total run time of $100 \mathrm{~min}$; autosampler injection volume of $1 \mu \mathrm{L}$; flame ionization detector (FID) temperature of $255^{\circ} \mathrm{C}$; injector port temperature of $250^{\circ} \mathrm{C}$; hydrogen flow rate of $40 \mathrm{~mL} / \mathrm{min}$; and air flow rate of $400 \mathrm{~mL} / \mathrm{min}$. Individual peaks were identified by comparison with known standards (Nu-Chek Prep, Inc., Elysian, MN, USA) that were designed to mimic RBC FA composition. Individual FA were quantified as a percent of total area under 
the peak. The internal standard (ISTD) was included with each GC run for adjustment of fatty acid time signatures.

\section{FRAP Assay}

Frozen plasma samples were defrosted to room temperature. Total antioxidant capacity was measured using FRAP assay adapted from the Benzie and Strain method. ${ }^{83}$ The FRAP reagent was prepared fresh before each series of measurements so that it contained $200 \mathrm{~mL}$ acetate buffer, (300mM, pH 3.6), 20 mL TPTZ solution (O.062 g TPTZ in 20 mL 40mM HCl), $20 \mathrm{~mL} \mathrm{FeCl}_{3}$ solution (0.2748 $\mathrm{g} \mathrm{FeCl}_{3}-6 \mathrm{H}_{2} 0$ in $50 \mathrm{~mL}$ DDW), and $24 \mathrm{~mL}$ DDW. A 20- $\mu \mathrm{L}$ aliquot of plasma was added to $60 \mu \mathrm{L}$ DDW and $1000 \mu \mathrm{L}$ FRAP reagent, vortexed, and incubated at room temperature for $4 \mathrm{~min}$. The absorbance was determined at $593 \mathrm{~nm}$ against a blank consisting of $1000 \mu \mathrm{L}$ FRAP reagent and $80 \mu \mathrm{L}$ DDW. This procedure was performed in triplicate for each study participant. Calibrations were set at a standard curve using a series of standards from diluting $1 \mathrm{mM}$ ferrous sulphate solution (0.278 g FeSO $4 \mathrm{H}_{2} 0$ in 1 liter DDW). The series of dilutions included concentrations of $0.1,0.2,0.4,0.6,0.8$, and 1.0 mM. FRAP values for each serum sample was determined by UV-visible spectrophotometer (BioMate 3S; Thermo Scientific, Waltham, MA, USA). An average FRAP value was determined for each study participant from the triplicate serum samples measured. These individual FRAP values were then averaged to determine an overall mean for each case status group. 


\section{$\underline{\text { Statistical Analysis }}$}

Descriptive statistics as well as all subsequent analyses were performed in SPSS version 21 (IBM Corp., Armonk, NY, USA). General linear models (GLM) were constructed for all statistical analyses. To determine if individual FRAP values differed between case status, BMI and current tobacco use were used as covariates in the model because current literature suggests that these factors are associated with increased oxidative stress ${ }^{84-87}$ Although evidence exists associating other patient characteristics such as age ${ }^{88}$, hypercholesterolemia ${ }^{84}$, diabetes ${ }^{85}$, and hypertension ${ }^{89,90}$ with an increase in oxidative stress, these variables were not included as covariates due to limited sample size. The GLMs conducted for individual RBC fatty acids were performed using only case status as a factor/treatment effect to determine significance between cases and controls. Covariates were not used in the GLMs predicting individual fatty acids by case status because some fatty acids were found in RBC membranes of only a few individuals. GLMs were also performed predicting overall levels of fatty acid types and fatty acid ratios based on case status after adjusting for BMI and dyslipidemia. These 2 covariates were chosen for the models due to evidence suggesting that overweight/obesity (BMI > 25) and dyslipidemia are major contributors to risk of CHD. ${ }^{91,92}$ In order to determine the association between RBC omega-3 fatty acid content and FFQ daily omega-3 fatty acid consumption, a GLM was constructed using the overall average omega- 3 content from food sources self-reported in the FFQ as a covariate. A $p$ value of $<0.05$ was considered statistically significant for all analyses. 


\section{CHAPTER 4}

\section{RESULTS}

\section{$\underline{\text { Patient Characteristics }}$}

Of the 38 participants for whom patient characteristics were obtained, 2 participants failed to respond to all of the questions inquiring about demographics and CVD risk factors (e.g. status of diabetes was not indicated and family history was not known due to adoption), and 7 participants did not have lipid profile information. Consequently, descriptive statistics reflect samples of varying size as shown in Table 1. Ethnicity and race were excluded from the demographics due to lack of ethnic diversity in the 2 groups. Average BMI of the control group was $31.45 \pm 1.49$ and $31.49 \pm 2.11$ for the case group, indicating a nonsignificant difference in height and weight between the 2 groups. However, based on BMI value both groups were considered moderately obese (Obesity Class 1: 30-34.9). A BMI value of $>25$ and physical inactivity is considered a CHD risk factor and was included in the analysis of patient characteristics. Although physical activity did not significantly differ between case and controls, frequency and duration of physical activity for participants who reported that they are currently physically active were included for additional information. Presence of diabetes was more prominent in the control group compared to the case group. Current tobacco use, family history of CAD, hypertension, and dyslipidemia was higher in the case group compared to controls, despite the lower sample size. Total and LDL cholesterol did not significantly differ between the cases and controls; however, HDL cholesterol was significantly lower, while triglycerides were significantly higher in the case group compared to the controls. In addition, primary reasons for cardiac catheterization of both case and control groups included ST-elevation myocardial 
infarction (STEMI) (n=2), non-ST-elevation myocardial infarction (NSTEMI) (n=5), abnormal stress ( $n=15)$, unstable angina $(n=1)$, chest pain $(n=11)$, cardiomyopathy $(n=1)$, and severe mitral regurgitation $(M R)(n=1)$. 
Table 1: Characteristics of Study Participants

\begin{tabular}{|c|c|c|}
\hline & Control & Case \\
\hline \multicolumn{3}{|l|}{ Demographics } \\
\hline Age (yrs), (mean \pm SEM), $n$ & $59 \pm 2,29$ & $53 \pm 3,9$ \\
\hline \multicolumn{3}{|l|}{ Gender, $n(\%)$} \\
\hline Male & $14(48.3 \%)$ & $6(66.6 \%)$ \\
\hline Female & $15(51.7 \%)$ & $3(33.3 \%)$ \\
\hline \multicolumn{3}{|l|}{ CVD Risk Factors } \\
\hline \multicolumn{3}{|l|}{ Family Hx of CAD, $n(\%)$} \\
\hline Yes & 19 (65.5\%) & $7(87.5 \%)$ \\
\hline No & $10(34.4 \%)$ & $1(12.5 \%)$ \\
\hline \multicolumn{3}{|l|}{ Diabetes Mellitus, $n$ (\%) } \\
\hline Yes & $8(27.5 \%)$ & $1(12.5 \%)$ \\
\hline No & $21(72.5 \%)$ & $7(87.5 \%)$ \\
\hline \multicolumn{3}{|l|}{ Hypertension, $n(\%)$} \\
\hline Yes & $19(65.6 \%)$ & $6(66.6 \%)$ \\
\hline No & $10(34.4 \%)$ & $3(33.3 \%)$ \\
\hline \multicolumn{3}{|l|}{ Dyslipidemia, $n$ (\%) } \\
\hline Yes & 15 (51.7\%) & $6(66.6 \%)$ \\
\hline No & $14(48.2 \%)$ & $3(33.3 \%)$ \\
\hline \multicolumn{3}{|l|}{ Current Tobacco Use, $n(\%)$} \\
\hline Yes & $7(24.1 \%)$ & $6(66.6 \%)$ \\
\hline No & $22(75.9 \%)$ & $3(33.3 \%)$ \\
\hline \multicolumn{3}{|l|}{ BMI $>25, n(\%)$} \\
\hline Yes & $23(79.3 \%)$ & $8(88.9 \%)$ \\
\hline No & $6(20.7 \%)$ & $1(11.1 \%)$ \\
\hline \multicolumn{3}{|l|}{ Physical Activity, $n$ (\%) } \\
\hline Yes & $18(62.1 \%)$ & $5(55.5 \%)$ \\
\hline No & $11(37.9 \%)$ & $4(44.4 \%)$ \\
\hline \multicolumn{3}{|l|}{ Frequency } \\
\hline Once/wk & $1(5.5 \%)$ & \\
\hline Twice/wk & $4(22.2 \%)$ & $1(20 \%)$ \\
\hline 3 times/wk & $6(33.3 \%)$ & $2(40 \%)$ \\
\hline$>4$ times/wk & $7(38.9 \%)$ & $2(40 \%)$ \\
\hline \multicolumn{3}{|l|}{ Duration } \\
\hline$<30 \mathrm{~min} / \mathrm{d}$ & $3(16.7 \%)$ & $2(40 \%)$ \\
\hline $30-45 \mathrm{~min} / \mathrm{d}$ & $7(38.9 \%)$ & $2(40 \%)$ \\
\hline $60-90 \mathrm{~min} / \mathrm{d}$ & $3(16.7 \%)$ & \\
\hline$<90 \mathrm{~min} / \mathrm{d}$ & $5(27.7 \%)$ & $1(20 \%)$ \\
\hline \multicolumn{3}{|l|}{ Lipids } \\
\hline Cholesterol (mg/dL), (mean \pm SEM) & $161.6 \pm 9.7$ & $166.1 \pm 11.6$ \\
\hline HDL $(\mathrm{mg} / \mathrm{dL}),($ mean \pm SEM) & $40.5 \pm 1.8$ & $34.7 \pm 2.6$ \\
\hline LDL $(\mathrm{mg} / \mathrm{dL}),($ mean \pm SEM $)$ & $94.08 \pm 8.7$ & $96.3 \pm 10.9$ \\
\hline Triglycerides $(\mathrm{mg} / \mathrm{dL}),($ mean \pm SEM) & $142.1 \pm 16.6$ & $193 \pm 30.4$ \\
\hline
\end{tabular}




\section{$\underline{\text { Red Blood Cell Fatty Acid Composition }}$}

Fatty acid composition for cases and controls is shown in Table 2. Each individual fatty acid is expressed as average percent area under the total chromatogram. An overall average of saturated, MUFA, and PUFA of both case and control groups is also shown in Table 2 in addition to ratios between each fatty acid type. The GLM analyses did not indicate significant difference of individual fatty acids by case status with the exception of palmitic acid $(16: 0)(p=$ 0.018), as depicted in Figure 1. Table 2 also illustrates the results of GLMs predicting individual fatty acid levels by case status. F-ratios and degrees of freedom for all models can be found in Appendix A. EPA (22:4, n-3), nervonic acid (24:1, n-9), and lignoceric acid (24:0, saturated) were found in very few individuals ( $n=1 ; n=2 ; n=3$, respectively); therefore, these fatty acids were excluded from the analyses due to lack of statistical power. GLM analyses predicting average levels of fatty acid types and fatty acid ratios by case status did not indicate significant treatment effect; however, the model predicting average omega-6 content in RBC membranes found a marginally nonsignificant effect of case status ( $p=0.079)$, while BMI and dyslipidemia were significant covariates $(p=0.014 ; p=0.009)$. The overall corrected model for average omega-9 RBC content was marginally nonsignificant $(p=0.089)$. The majority of the explained variance was associated with BMI as a covariate $(p=0.018)$. The results of all the corrected GLMs can be found in Appendix B. Figure 2 depicts the average omega-6 PUFA content of RBC membranes in case and control status. 
Table 2: Fatty acid profiles of RBC membranes for controls and cases ${ }^{1}$

\begin{tabular}{|r|r|r|r|}
\hline \multicolumn{1}{|l|}{ Fatty Acids } & \multicolumn{1}{l|}{ Controls } & $\boldsymbol{p}$-value \\
\hline Myristic, 16:0 & $2.38 \pm 1.12 ; 14$ & $4.23 \pm 3.25 ; 4$ & 0.498 \\
\hline Palmitic, 16:0 & $24.51 \pm 0.47 ; 29$ & $21.78 \pm 1.29 ; 9$ & $0.018^{*}$ \\
\hline Palmitoleic ,16:1 & $2.92 \pm 0.39 ; 26$ & $4.51 \pm 1.6 ; 9$ & 0.171 \\
\hline Stearic, 18:0 & $21.76 \pm 0.48 ; 29$ & $22.08 \pm 0.73 ; 9$ & 0.407 \\
\hline Oleic,18:1 & $14.57 \pm 0.32 ; 29$ & $13.91 \pm 0.83 ; 8$ & 0.379 \\
\hline Linoleic, 18:2 & $12.94 \pm 0.49 ; 29$ & $12.05 \pm 0.76 ; 9$ & 0.373 \\
\hline DGLA, 20:3 & $5.16 \pm 3.89 ; 2$ & $0.696 \pm 0 ; 1$ & 0.626 \\
\hline Arachidonic, 20:4 & $19.25 \pm 0.51 ; 29$ & $20.18 \pm 1.42 ; 8$ & 0.448 \\
\hline EPA, 20:5 & - & $2.56 \pm 0 ; 1$ & - \\
\hline Adrenic, 22:4 & $3.45 \pm 0.34 ; 26$ & $4.12 \pm 0.33 ; 9$ & 0.294 \\
\hline DPA, 22:5, n-3 & $0.82 \pm 0.24 ; 2$ & $1.43 \pm 0.21 ; 4$ & 0.151 \\
\hline Lignoceric, 24:0 & $0.96 \pm 0.16 ; 3$ & & - \\
\hline DHA, 22:6 & $1.92 \pm 0.39 ; 8$ & $2.28 \pm 1.24 ; 2$ & 0.719 \\
\hline Nervonic, 24:1 & $1.94 \pm 0.26 ; 2$ & & - \\
\hline Fatty Acid Types \& & Controls (n $=29)$ & Cases (n= 9) & $\boldsymbol{p}$-value \\
Ratios & & & \\
\hline Saturated & $18.92 \pm 0.80$ & $19.53 \pm 1.50$ & 0.961 \\
\hline n-9 PUFA & $13.71 \pm 0.56$ & $13.81 \pm 0.74$ & 0.089 \\
\hline n-7 PUFA & $2.62 \pm 0.39$ & $3.06 \pm 1.25$ & 0.200 \\
\hline n-6 PUFA & $12.38 \pm 0.50$ & $11.08 \pm 0.80$ & 0.006 \\
\hline n-3 PUFA & $0.54 \pm 0.18$ & $0.72 \pm 0.32$ & 0.557 \\
\hline MUFA: PUFA & $0.81 \pm 0.07$ & $0.91 \pm 0.12$ & 0.798 \\
\hline n-6: n-3 & $2.63 \pm 0.05$ & $1.22 \pm 0.18$ & 0.464 \\
\hline Saturated: Unsaturated & & $4.06 \pm 1.79$ & 0.581 \\
\hline
\end{tabular}

${ }^{*} p$-value is significant at $<0.05$

${ }^{1}$ Fatty acids are expressed by average area under the peak and reported as mean \pm SEM; $n$. 


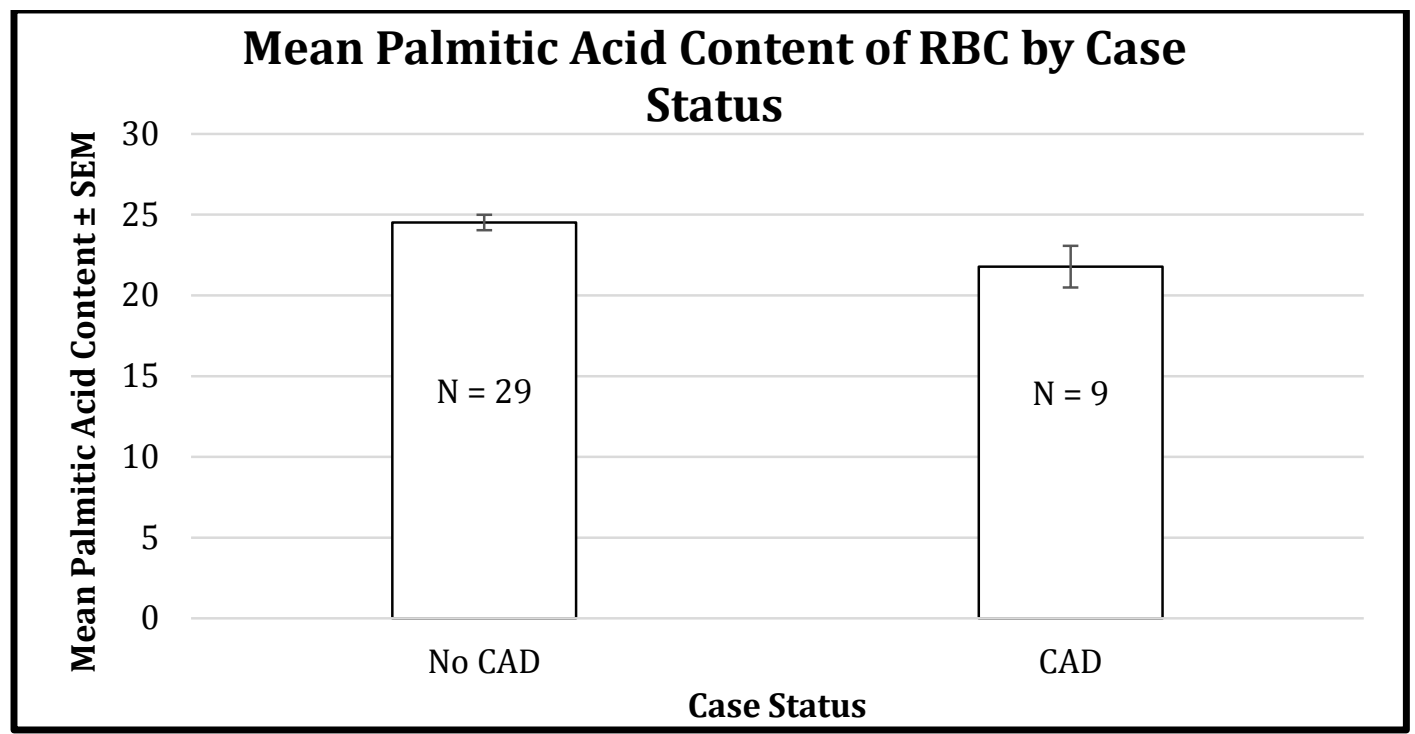

Figure 1: Average RBC palmitic acid content by control and case status

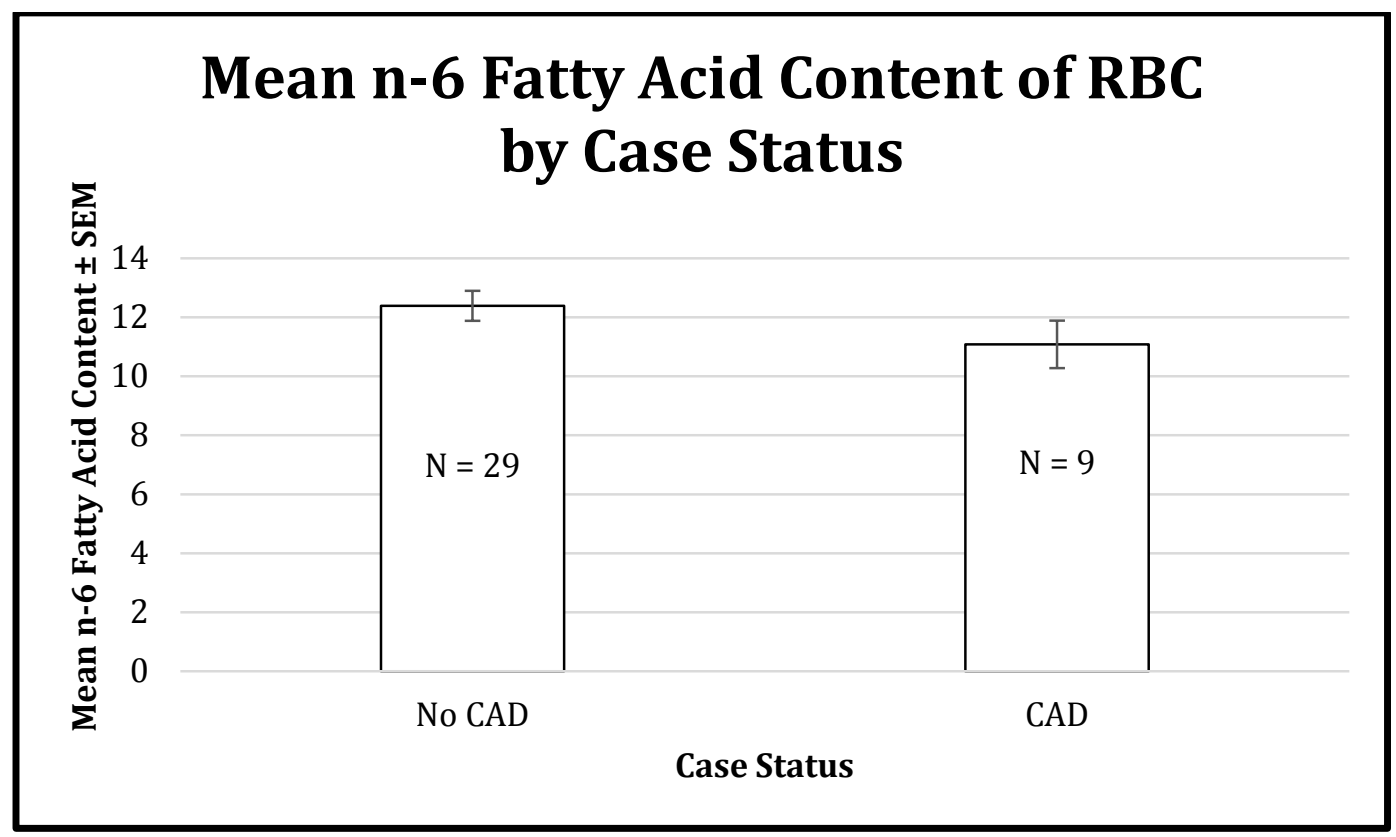

Figure 2: Average RBC omega-6 fatty acid level by control and case status

\section{Food Frequency Questionnaire Responses}

The overall average omega-3 content from EPA, DHA and ALA reported in the FFQ was $0.19 \pm 0.06 \mathrm{~g} / \mathrm{d}$ and $0.15 \pm 0.06 \mathrm{~g} / \mathrm{d}$ for the controls and cases, respectively. Table 3 further describes the content of each individual omega-3 PUFA as determined by FFQ responses. 
Portion size for gender was included in the calculation of the daily consumption of omega-3 fatty acids. The GLM analyzing case and control differences in average RBC omega-3 content with the average FFQ-estimated omega-3 intake as a covariate did not indicate a significant association $(p=0.524)$. This relationship between these variables is depicted in Figure 3 . The regression line is not indicated in Figure 3 because the GLM analysis indicates that the regression line does not significantly differ from a null (empty) model. Figure 4 depicts the fish consumption for cases and controls that were self-reported in the questionnaire. Figure 5 depicts the percentage of each case group in categories of self-reported fish consumption frequency in the past 6 months. Figure 6 depicts the self-reported consumption of walnuts and flaxseeds for cases and controls. Figure 7 depicts the frequency of consumption for those who reported intake of walnuts in the past 6 months. Figure 8 depicts the usage and consumption of canola oil, flaxseed oil, and cod liver oil self-reported in the FFQ for cases and controls. Figure 9 depicts the frequency of consumption for those who reported intake of canola oil in the past 6 months. Differences in frequency of flaxseeds, flaxseed oil, and cod liver oil consumption between the cases and controls were not calculated due to a low number of responses. It was found that $17.2 \%(n=5)$ of the control group and 33.3\% $(n=3)$ of the case group reported use of omega-3 PUFA/fish oil supplements, as depicted in Figure 10. The use of the dietary supplements was not included in the estimated daily food consumption of omega-3 PUFA due to repeated responses of uncertain supplement brand and dosage among participants.

Table 3: Average ALA, EPA, DHA consumed as reported in FFQ

\begin{tabular}{|r|r|r|}
\hline Omega-3 PUFA & Control (n=29) & Case (n=9) \\
\hline ALA, 18:3 g/d & $0.41 \pm 0.18$ & $0.30 \pm 0.14$ \\
\hline EPA, 20:5 g/d & $0.58 \pm 0.17$ & $0.02 \pm 0.01$ \\
\hline DHA, 22:6 g/d & $0.15 \pm 0.03$ & $0.06 \pm 0.03$ \\
\hline
\end{tabular}




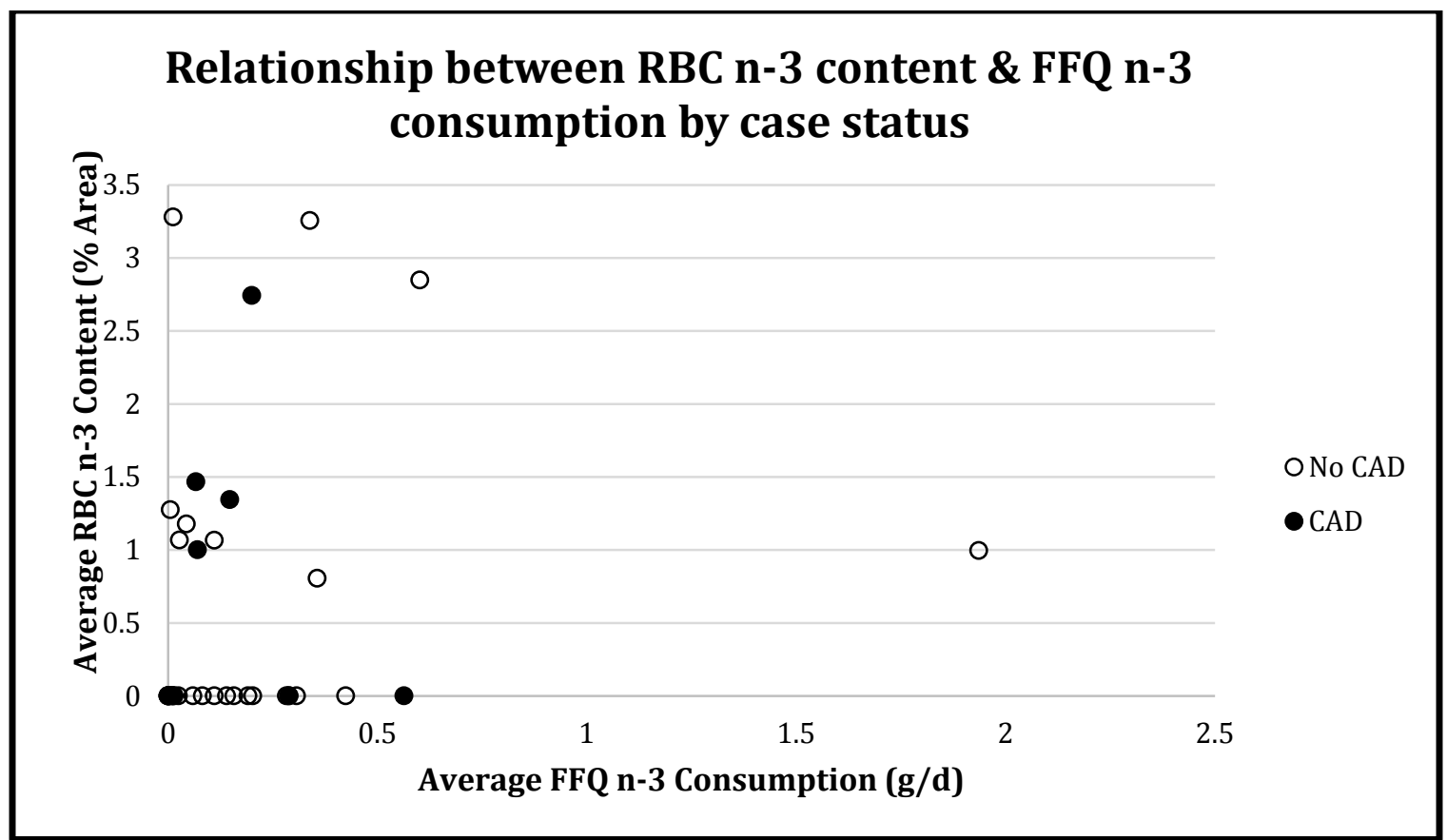

Figure 3: Correlation between average RBC omega-3 content and average FFQ omega-3 consumption by control and case status

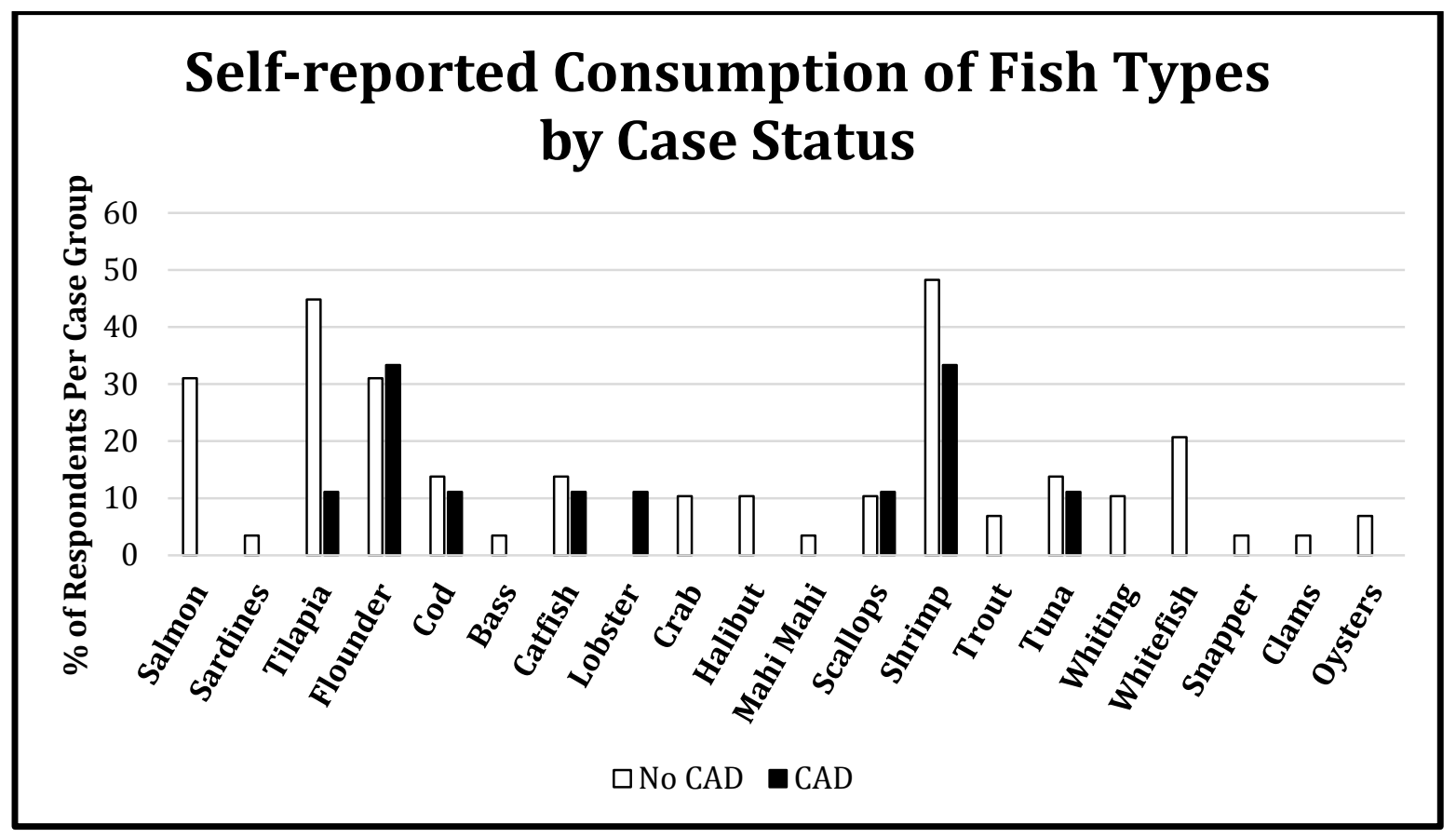

Figure 4: Fish consumption reported in FFQ for control and case status 


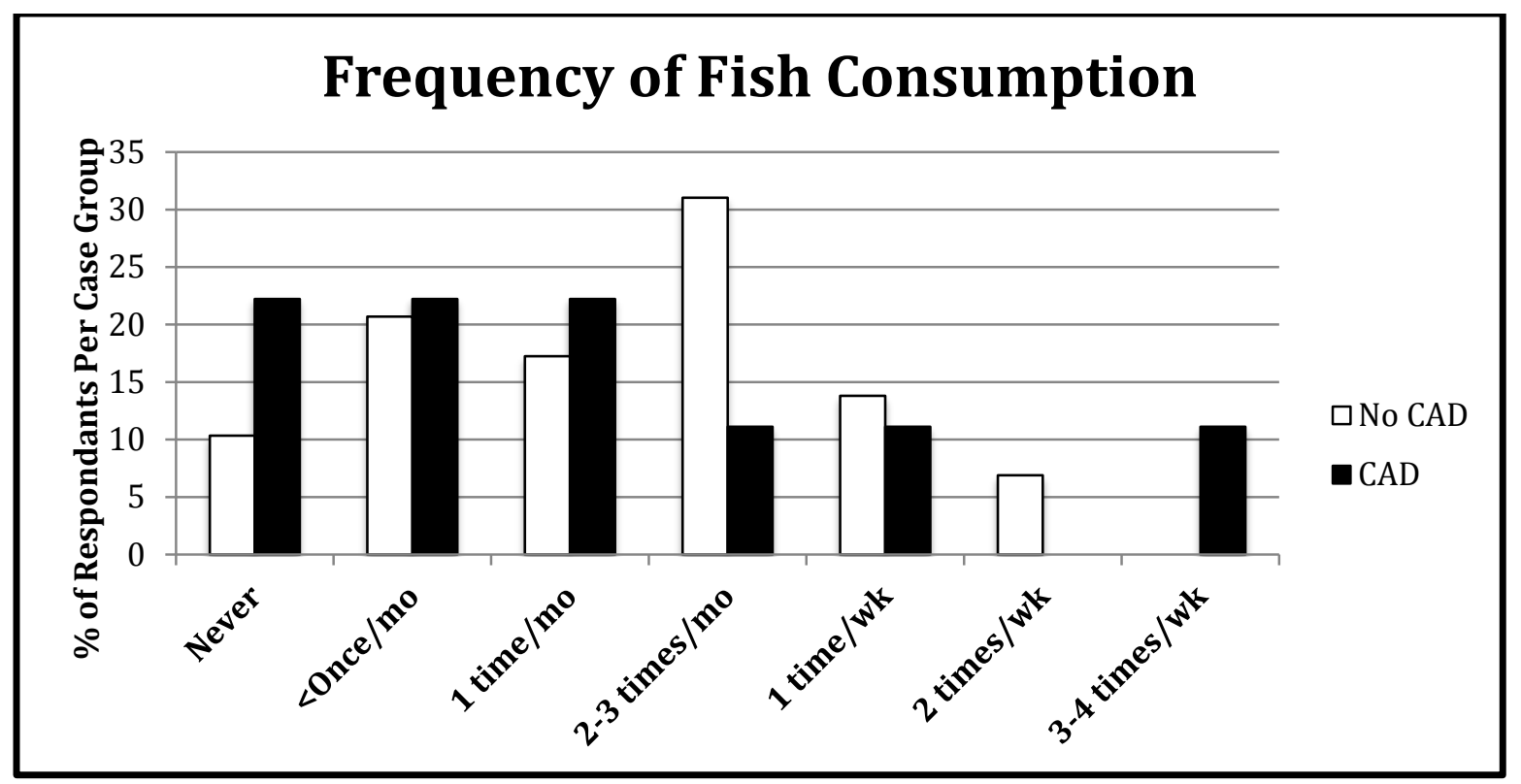

Figure 5: Frequency of fish consumption in past 6 months reported in FFQ by control and case status

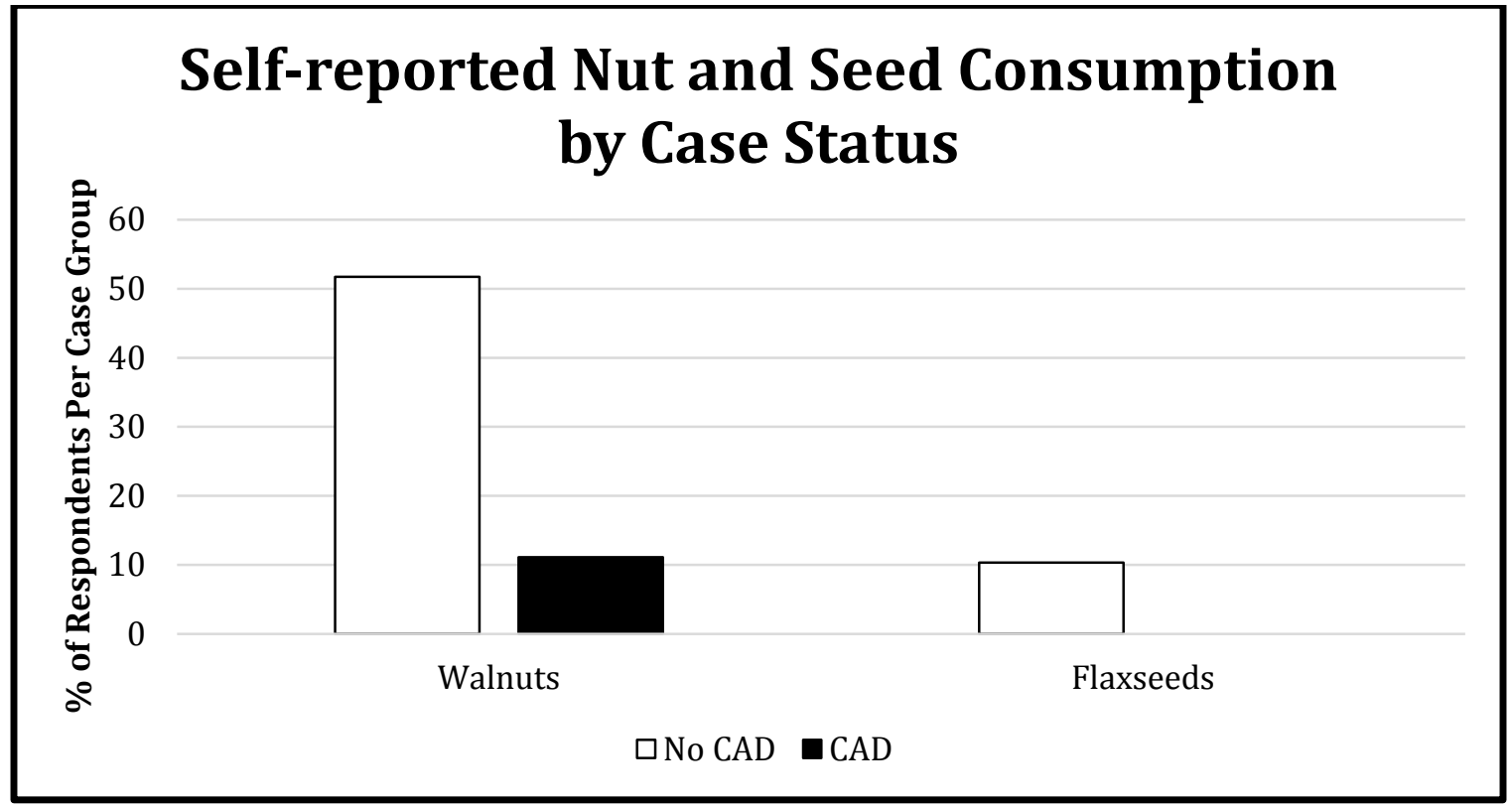

Figure 6: Walnut and flaxseed consumption reported in FFQ for control and case status 


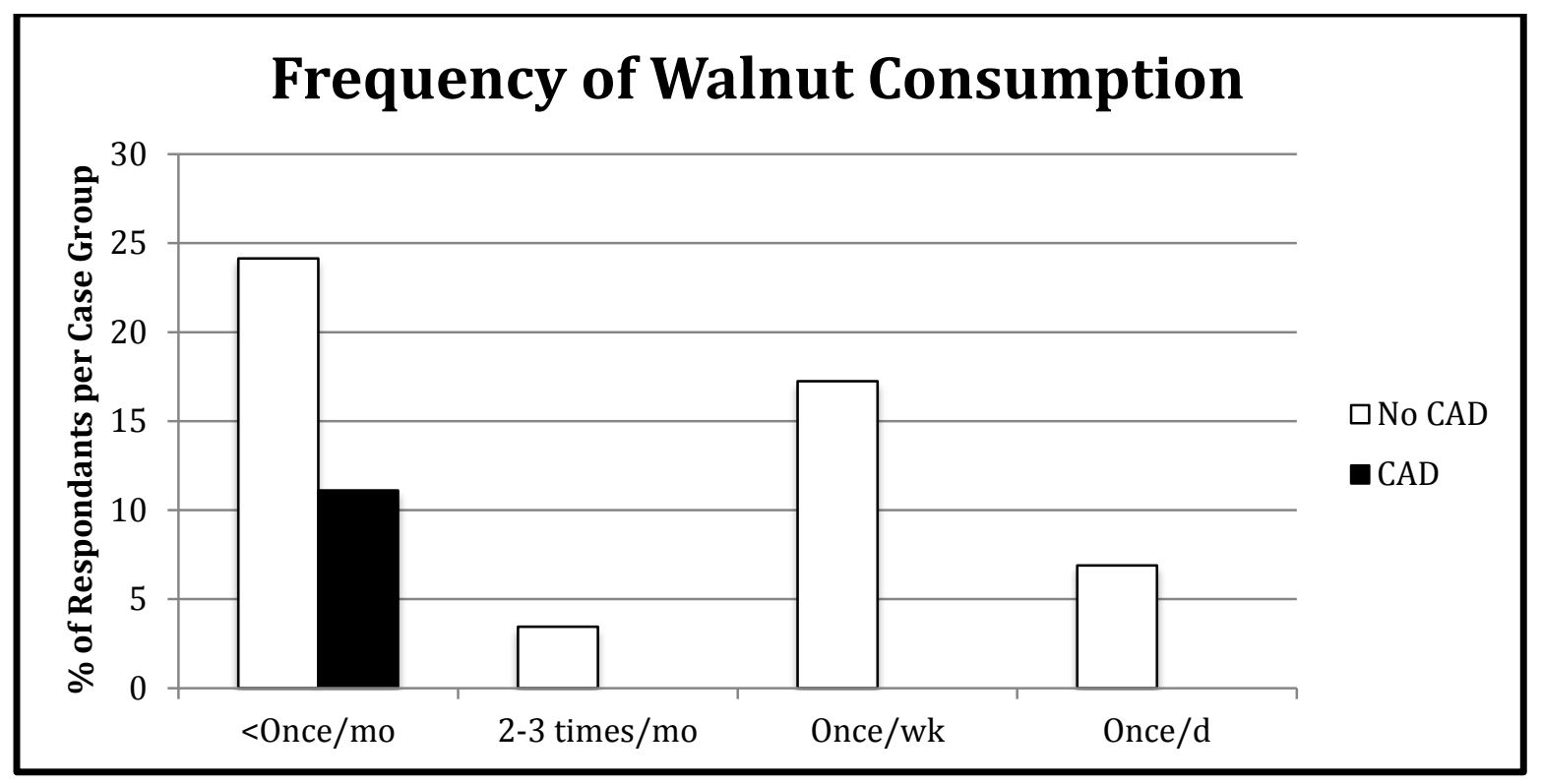

Figure 7: Frequency of walnut consumption in the past 6 months reported in FFQ for control and case status

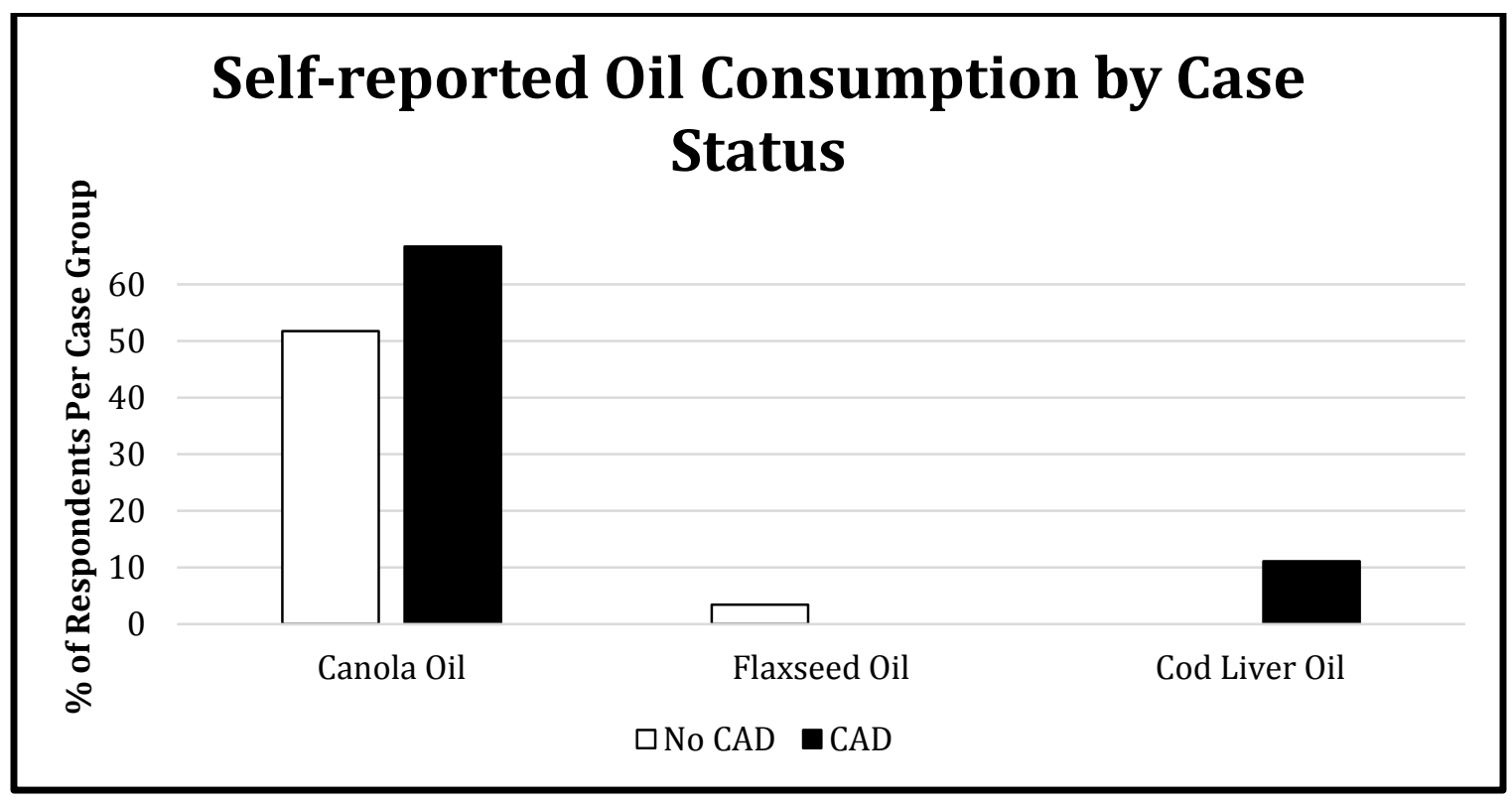

Figure 8: Canola oil, flaxseed oil, and cod liver oil consumption reported in FFQ for control and case status 


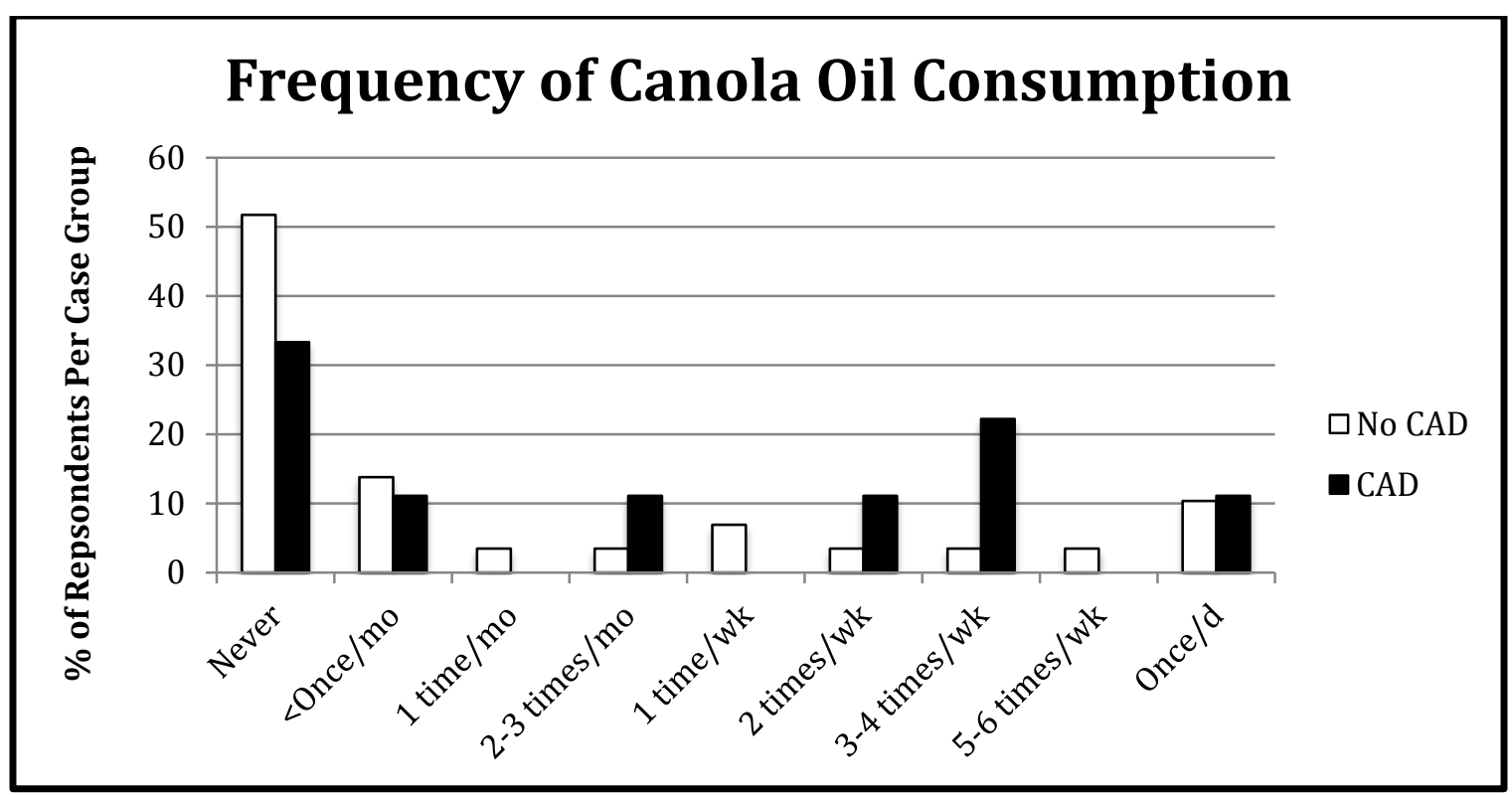

Figure 9: Frequency of canola oil consumption in past 6 months reported in FFQ by control and case status

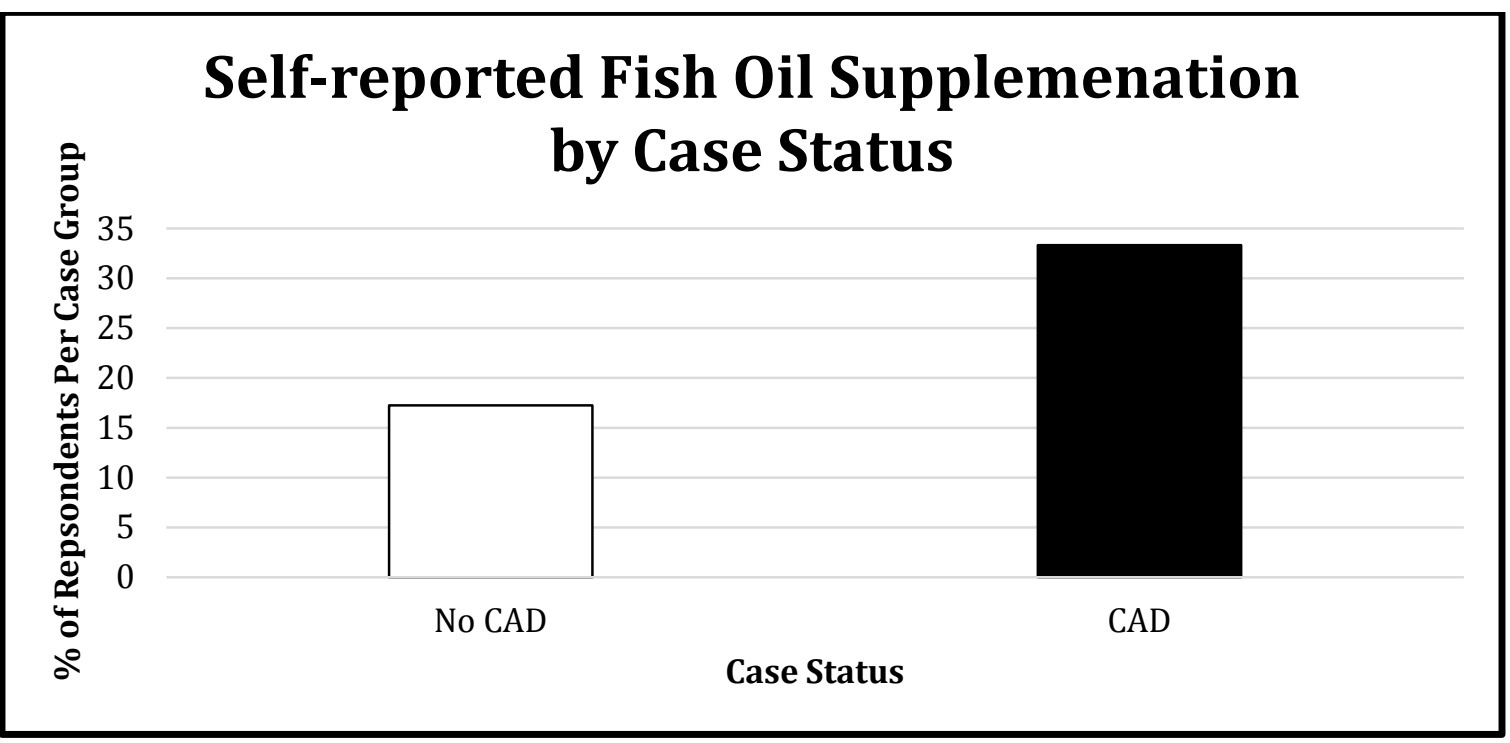

Figure 10: Omega-3 supplement usage reported in FFQ for control and case status 


\section{FRAP Assay}

A total of 39 FRAP assays were conducted. The average concentration of FRAP values for the control group $(n=29)$ was $0.594 \pm 0.019 \mu \mathrm{mol} / \mathrm{L}$, while the average FRAP value for the case group $(\mathrm{n}=9)$ was $0.659 \pm 0.489 \mu \mathrm{mol} / \mathrm{L}$ (mean $\pm \mathrm{SEM})$. The overall corrected model for predicting FRAP value by case status with BMI and current tobacco use as covariates was found to be nonsignificant $(p=0.390)$. Additional model statistics of FRAP can be found in Appendix B. Figure 11 depicts the average FRAP value by case status.

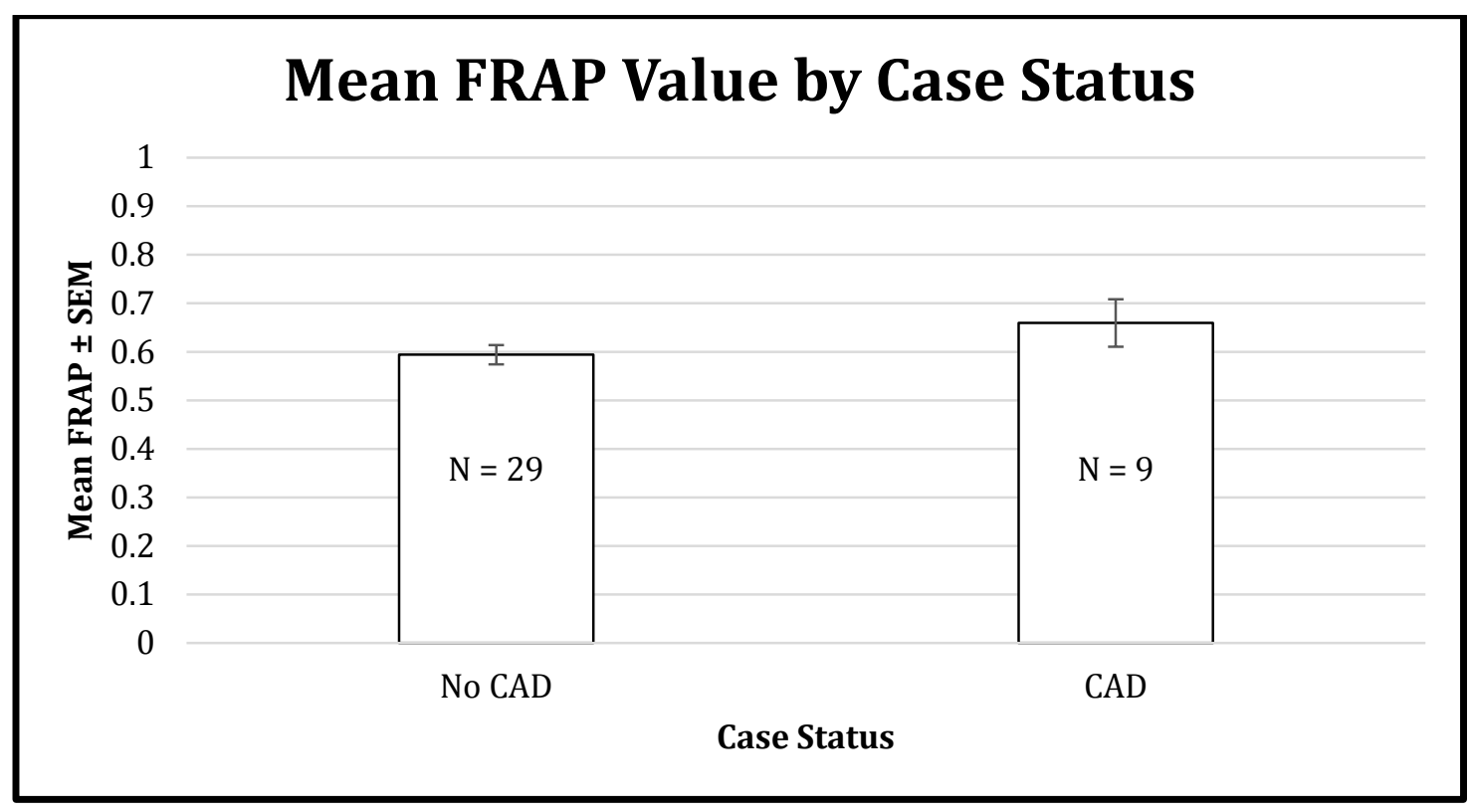

Figure 11: Average FRAP value by control and case status 


\section{CHAPTER 5}

\section{DISCUSSION}

\section{$\underline{\text { Red Blood Cell Fatty Acids and Dietary Intake }}$}

It is clear that deficiencies and imbalances of saturated fatty acids, MUFA, and PUFA within the diet and composition of cell membranes propose a significant risk for development of cardiovascular diseases. The recent suggestion of fatty acid composition within the RBC membrane as an additional CHD risk factor brings into question the nature of the differences of fatty acid profiles between those with and at risk for CAD. In the present study, 6 particular fatty acids (palmitic, 16:0; palmitoleic, 16:1; stearic, 18:0; oleic, 18:1; linoleic, 18:2; arachidonic, 20:0; and adrenic acid, 22:4) were found in the RBC membranes of all participants. Additionally, these fatty acids were the most abundant fatty acids in RBC membranes of cases and controls, as indicated by considerably greater percent area under the peak. However, the majority of fatty acids present in RBC membranes, with the exception of palmitic acid (16:0, saturated), did not differ significantly between case and control groups. It is still of interest to acknowledge the amount of each fatty acid present in RBC membranes despite a nonsignificant correlation with case status. For instance, MUFA such as oleic acid (18:1, n-9) have previously been shown to increase the risk of acute nonfatal $\mathrm{MI}^{69}$ and other ACS events ${ }^{66}$. Similarly, RBC palmitoleic acid $(16: 1, n-7)$ has been reported as having a positive relationship with CAD. ${ }^{93}$ Inverse relationships with ACS case status with increasing RBC levels of palmitoleic and stearic acid (18:0, saturated) have also been observed. ${ }^{6}$ Due to the limited sample size of this study, it is unclear whether the lack of association between RBC fatty acids (except palmitic) and CAD risk are representative of the population or a result of limited statistical power. 
The significance of the correlation between RBC palmitic acid and case status indicated that a decreased level of this saturated fatty acid was associated with CAD. This finding is opposite of the long-standing dietary recommendation that limiting saturated fat is essential in preventing CVD. Until recently the association of saturated fat and CHD has remained unquestioned. The majority of clinical trials investigating the relationship of reduced saturated fat and CHD risk have been achieved by increasing the amount of replacement nutrients such as MUFAs or PUFAs. ${ }^{94}$ Therefore, the positive effects of clinical trials may be inaccurate due to the confounding effects of these replacement nutrients. Additionally, relatively few single-nutrient clinical trials have investigated the relationship between reducing saturated fat intake and CHD. ${ }^{95}$ A meta-analysis from 21 epidemiologic studies, as well as a subgroup analysis among 15 of those studies, did not find a significant association with saturated fat intake and risk for CHD, stroke, or CVD. ${ }^{94}$ This nonsignificant association of saturated fat and CVD was further supported by another systematic review. ${ }^{95}$ It should also be taken into consideration the relative amount of saturated fatty acids compared to the amount of unsaturated fatty acids present in the RBC membrane. Although a nonsignificant correlation was seen with case status and ratio of saturated to unsaturated fatty acids, previous findings have shown an inverse association with CHD risk when amount of PUFA were higher than that of saturated fat. ${ }^{96} \mathrm{~A}$ larger sample size of the present study is needed to determine whether overall saturated fat content and/or saturated to unsaturated fatty acid ratios are indeed different between those with and at risk for CAD.

The relatively ample amount of linoleic acid (18:2, n-6) present in both case and control groups was not of surprise due to the abundance of this EFA in the Western diet. ${ }^{12,68}$ However, it was of considerable surprise that ALA $(18: 3, n-3)$ was not present in RBC membranes of all study participants. This may be due to the immediate metabolism of this EFA to longer-chain 
omega-3 PUFA and if ALA is stored within cell membranes it is primarily stored as DHA (22:6, n-3). ${ }^{42}$ With that being said, DHA was present in both cases and controls, which lends a possible explanation for the absence of ALA in RBC membranes. Blood levels of ALA would thereby be dependent on recent dietary consumption. Therefore, RBC membranes may not provide an accurate representation of ALA intake because they reflect long-term dietary fat consumption. Findings from a recent systematic review ${ }^{95}$ did not support an association between dietary ALA and CHD, which are inconsistent with previous reports of reduced risks of SCD. ${ }^{20,42,97}$

Moreover, reports of association with CHD risk and the principal omega-6 PUFA, linoleic acid, remain inconsistent. ${ }^{68,69}$ The RBC content of the most abundant omega-6 fatty acids, linoleic (18:2) and arachidonic acid (20:4), found in the present study did not provide evidence for a relationship with CAD. However, the overall average of omega-6 PUFA had a marginally nonsignificant effect on case status, suggesting that individuals who do not have CAD were consuming more omega- 6 fatty acids in their diet. This finding further supports the large body of literature that suggests higher intakes of omega-6 PUFA reduce the risk for CHD. ${ }^{98}$ There have been recent arguments based on the assumption that reducing intake of linoleic acid should reduce arachidonic acid content in tissues, thereby reducing the inflammatory potential of a high omega-6 intake and result in a lower risk for $\mathrm{CHD} .{ }^{98}$ However, there is limited evidence that linoleic acid exhibits a direct proinflammtory and proatherogenic effect in humans. ${ }^{98}$ Higher intakes of omega-6 PUFA have been reported as safe and to reduce intake to lower than the 5$10 \%$ of energy intake recommendation may be more likely to increase rather than decrease the risk for CHD. ${ }^{98}$

Similarly, omega-3 fatty acids have been largely shown to reduce the risk of CHD, atherosclerotic disease progression and cardiac events including mortality, nonfatal MI, and 
nonfatal stroke. ${ }^{13}$ The omega-3 fatty acids present in the RBC membrane of cases and controls, DHA (20:6) and EPA (20:5), and overall omega-3 fatty acid content were found to be nonsignificant indicators of case status. Although the content of DHA was slightly higher in the case group and EPA was only present in the RBCs of cases, the limited sample size in this study hinders the ability to provide sufficient evidence for a strong relationship with CAD. Previous reports have indicated that the amount of long-chain omega-3 fatty acids (EPA+DHA) in RBC membranes decrease with an increasing BMI value independent of reported fish intake ${ }^{77}$ and current tobacco use ${ }^{65}$. It was also found that the case group had a higher n-6: n-3 ratio than the controls ( $4.06 \pm 1.79$ vs. $2.63 \pm 0.875$ ), indicating that the case group was consuming more omega- 6 fatty acids compared to omega- 3 fatty acids. The optimal ratio of n-6: $n-3$ is recommended to be $2-4: 1^{8,12}$ which suggests that both the case and control groups were consuming the optimal amount of these EFA. This indicates why the ratio of n-6: n-3 PUFA was not considered a significant predictor of case status. This finding was of particular surprise due to reports that $60 \%$ of the adult population consume an n-6: n-3 ratio of $8-12: 1{ }^{12}$ Moreover, it is important to note that many individuals were excluded from the n-6: n-3 ratio analyses due to lack of omega-3 PUFA in their RBC membranes. This indicates that majority of this population are indeed deficient in omega-3 PUFA, a finding that is consistent with the lack of omega-3 fatty acids and overabundance of omega- 6 fatty acids found in the Western diet. ${ }^{8,12}$ A larger scale study is needed to determine whether these results are representative of this target population or a consequence of limited sample size.

In addition, a nonsignificant correlation was found between RBC omega-3 fatty acid content and self-reported intake of omega-3 fatty acids regardless of case status. This may be due to an inaccurate representation of daily omega-3 intake of participants because use of omega-3 
fatty acid supplementation was excluded. The FFQ used in this study has shown significant correlations of EPA and DHA intake and their respective mean plasma levels, but not for ALA intake and plasma levels. ${ }^{82}$ Although in the present study the use of this FFQ was used to correlate dietary intake of EPA, DHA, and ALA with RBC content of these omega-3 FA, rather than plasma, it should still remain of consideration that this questionnaire may not effectively estimate ALA intake. Previous studies have shown similar findings of no ${ }^{99}$ or modest $^{100}$ correlations with FFQ-estimated ALA intake and RBC ALA composition. However, few studies have indicated that dietary assessments provide reasonable estimates of EPA and DHA content in RBC membranes. ${ }^{64,77}$ Sun et al. ${ }^{64}$ suggested that obtaining a long-term measure of omega-3 PUFA intake through use of repeated FFQs can further strengthen correlations with RBC omega3 fatty acids by reducing self-reported bias. It is important to note that even accurate selfreported intake of omega-3 PUFA does not reflect inter-individual differences in fatty acid metabolism that may affect RBC membrane FA levels.$^{80}$ Despite these limitations, this FFQ provides a representation of dietary sources of omega-3 PUFA commonly consumed in this population, which may guide dietary recommendations for increasing omega-3 PUFA intake in this specific age, culture, and Southern Appalachian region.

It was also found that this particular population was consuming well below the recommended daily intakes of ALA and EPA+DHA. The control group was reportedly consuming $0.4 \mathrm{~g} / \mathrm{d}$ ALA and $0.1 \mathrm{~g} / \mathrm{d}$ EPA and DHA combined, while the case group was consuming $0.3 \mathrm{~g} / \mathrm{d}$ ALA and $0.04 \mathrm{~g} / \mathrm{d}$ EPA+DHA. Both groups were consuming well below the Acceptable Macronutrient Distribution Range (AMDR) for ALA, which is estimated to be 0.6 $1.2 \%$ of energy intake (based on a 2000-calories diet) or about $1.3-2.7 \mathrm{~g} / \mathrm{d}$ ALA. ${ }^{13}$ The AMDR for ALA is designed as a guideline for healthy individuals to consume omega-3 fatty acids with 
the intent to prevent EFA deficiency. The upper range of the AMDR corresponds to the amount of ALA commonly consumed in the US and should not be considered as an upper limit of this EFA. It has been suggested that the tolerable upper limit of ALA is $6.7 \mathrm{~g} / \mathrm{d}^{12,13}$ The exceedingly low intake of ALA observed in this study may be attributable to the infrequent consumption of walnuts, canola oil, flaxseeds, and flaxseed oil as reported in the FFQ.

Recommendations for healthy individuals (those without CHD) are $0.3-0.5 \mathrm{~g} / \mathrm{d}$ EPA+DHA; however, average intake of EPA+DHA in the US is only $0.1-0.2 \mathrm{~g} / \mathrm{d} .{ }^{13}$ This indicates that the reported consumption of EPA+DHA in the control group is representative of average long-chain omega-3 PUFA intake in the Western diet and US adult population. It has also been recommended by the American Heart Association (AHA) that individuals without CHD consume fatty fish (salmon, tuna, mackerel, etc.) at least twice per week and include foods and oils rich in ALA (flaxseeds, walnuts; canola, flaxseed, and soybean oils). ${ }^{13}$ It was found that majority of the control group 31\% (n=9) was consuming fish rich in omega-3 PUFA only 2-3 times/month, while only $22 \%(n=2)$ was meeting the twice per week recommendation. For individuals with CHD, AHA dietary recommendations include consuming about $1 \mathrm{~g}$ EPA+DHA/d, preferably from fatty fish. ${ }^{13}$ The Food and Drug Administration (FDA) has recommended an upper limit of EPA+DHA intake of $3 \mathrm{~g} / \mathrm{d} .^{101}$

In this study none of the individuals within the case group reported that they were consuming fatty fish twice per week, while most of the individuals $(67 \%, n=6)$ reported consuming once/month or less in a 6-month period. This finding explains the minimal amount of EPA+DHA consumed per day among the case group. Again, this may not be a true representation of EPA+DHA consumed among the case group because use of omega-3 supplements was excluded. However, it does provide an accurate representation of daily 
DHA+EPA intake solely from food sources and suggests that this target population cannot consume the recommended 1g/d EPA+DHA through diet alone. Thus, individuals with CHD may benefit greatly from consuming an omega-3 fatty acid supplement, especially for those who have limited access to fish, limited financial means to purchase fish, fish allergens, or those who simply do not consume fish.

\section{$\underline{\text { Anthropometrics and Lifestyle Behaviors }}$}

It was discovered that family history, hypertension, dyslipidemia, current tobacco use, and a BMI value of 25 or greater were more prominent CVD risk factors in the case group compared to the controls. With the exception of BMI and dyslipidemia, these CVD risk factors were not used to predict case status due to the vast amount of literature indicating that these factors largely contribute to the development of heart disease. However, these factors were used as a means to determine whether major CVD risk factors are indeed representative of individuals with CAD. The higher prevalence of family history, hypertension, dyslipidemia, tobacco use, and overweight/obesity (BMI > 25) of those with CAD in this study supports the clinical utility of risks factors in evaluating increased risk(s) for heart disease.

The more prevalent CVD risk factors seen in the case group are considered to be modifiable risk factors except for family history, which is considered nonmodifiable (i.e. cannot be treated or controlled). Modifiable CVD risk factors include both lifestyle behaviors that can be changed as well as diseases and conditions that can be treated with diet, exercise, and/or medicine. ${ }^{1}$ According to a recent report from the World Health Organization (in collaboration with the World Heart Federation and World Stroke Organization), modifiable risk factors that contribute to the leading causes of death from CHD included tobacco use (9\%), increased blood glucose levels (6\%), physical inactivity (6\%), and overweight/obesity (5\%). ${ }^{102}$ Recently the 
AHA has developed a model of ideal cardiovascular health as a means to reduce the current AHA diet and nutrition guidelines to promote cardiovascular health and risk reduction. ${ }^{103}$ This concise model contains 4 lifestyle behaviors that promote optimal cardiovascular health such as tobacco avoidance, maintenance of a healthy body weight (BMI <25), participating in regular physical activity, and adhering to a healthy diet. ${ }^{103}$ Adopting all 4 of these lifestyle behaviors has been shown to result in an $80 \%$ risk reduction from all-cause mortality and protect against CHD. ${ }^{104}$ This significant risk reduction was achieved when study participants maintained these ideal behaviors for 7.6 years. ${ }^{104}$ It was also discovered that tobacco avoidance played the most substantial role in mortality and CHD risk reduction when compared to the other lifestyle behaviors. ${ }^{104}$ For instance, the survival rate of participants who smoked and adopted 2 or more behaviors remained significantly lower than nonsmoking, sedentary, and obese participants. ${ }^{104}$ This particular finding further contributes to evidence suggesting that tobacco avoidance prolongs life expectancy. ${ }^{104}$ Similarly, the higher prevalence of tobacco users observed in the case group of the present study demonstrates how tobacco usage is indeed a strong predictor of optimal cardiovascular health.

In addition, the higher prevalence of physical activity observed in the control group demonstrates the importance of participating in regular physical activity in regards to cardiovascular health. The lower prevalence of physical activity present in the case group may also explain why the cases had higher BMI values and were classified as overweight/obese. It was also discovered that the case group had higher plasma levels of LDL-cholesterol and triglycerides suggesting that the case group may be consuming a diet higher in saturated fat and cholesterol. The control group had higher HDL-cholesterol levels compared to the case group, which may be attributable to the higher prevalence of physical activity reported among the 
controls. However, it should be taken into consideration that additional factors could affect LDL, HDL and triglyceride levels such as age, gender, and genetics, all of which are nonmodifiable. ${ }^{1}$

As expected, it was found that individuals with CAD exhibited more CVD risk factors compared to individuals who were at risk for heart disease. The majority of risk factors present in the case group are modifiable, which lends promise that diet education and nutritional counseling may help individuals with CAD, as well as those at risk for CAD, in this Southern Appalachian region to establish healthier habits for improving cardiovascular health. Focusing on the 4 ideal lifestyle behaviors recommended by the AHA can not only lower the risk for $\mathrm{CHD}^{104}$ and cardiac death ${ }^{105}$ but also death from all causes ${ }^{104}$, which may increase the motivation of individuals to adopt these lifestyle modifications.

\section{$\underline{\text { Oxidative Stress }}$}

FRAP assay was chosen in this study to determine total antioxidant capacity between the 2 groups as a means to evaluate level of oxidative stress. FRAP assay has been considered a reliable and reproducible measurement of overall ability to resist oxidative damage via increased antioxidant levels in nonprotein samples (i.e. plasma). ${ }^{106}$ Surprisingly, higher FRAP values were found in the case group compared to controls, indicating a greater antioxidant capacity in those with CAD. Based on the literature, higher FRAP values have also been reported in case groups rather than controls for a variety of different disease states. ${ }^{106-109}$ Increased levels of FRAP can be explained by uric acid because uric acid has been shown to account for $60 \%$ of FRAP values seen in fresh human plasma. ${ }^{83,108}$ Thus, uric acid may interfere with the results of this study producing false positive readings of total antioxidant capacity in both cases and controls. Using a total antioxidant measurement in which uric acid is not confounded in the results should produce 
a more reliable and valid measure of oxidative stress. ${ }^{110}$ It was hypothesized that oxidative stress would be a significant predictor of case status due to the higher BMI values and smoking prevalence among cases compared to controls and the high-inflammatory component associated with CHD.

\section{$\underline{\text { Strengths and Limitations }}$}

Strengths of this study include detailed fatty acid analysis and clinical applicability due to the significance of palmitic acid and case status and the marginally nonsignificant association of omega-6 PUFA content with case status. Additional strengths include representation of usual omega-3 PUFA intake of individuals (>30 years) with and at risk for CAD in this Southern Appalachian region. This study also contributes to the growing body of literature assessing potential for RBC FA profiles as a predictive tool for CAD and provides methodology for standardization for future clinical use of RBC FA analysis. In addition, potential limitations should also be considered. Small sample size, particularly of the case group, limits the certainty of the statistical analyses in this study. A lack of incentive to provide study participants may have hindered the ability to acquire a larger sample size. Self-reporting bias likely limits the accuracy of the FFQ results as well as the omission of amount of omega-3 PUFA supplements being consumed. Even in the absence of reporting bias, inter-individual differences in digestion, absorption, genetic, and lifestyle factors result in varied RBC fatty acid levels in relation to dietary intake. Also, the FFQ only evaluated omega-3 PUFA intake. Dietary intakes of other fatty acids were not assessed and could have been correlated with RBC FA composition. It is possible that FRAP assay is confounded by uric acid content in plasma. Limited sample size prevented the use of additional covariates in the FRAP statistical model, despite their indication 
in the literature. Future studies need to address the limitations to validate the oxidative stress level and RBC membrane FA composition as a clinical predictor of CAD risk.

\section{Conclusion}

In conclusion, analysis of RBC membrane FA composition shows promise as a novel predictive tool in the clinical setting. Substantial differences were found in levels of the saturated fat, palmitic acid (16:0) and the overall content of omega-6 PUFA between individuals with and at risk for CAD. This suggests that there is indeed a plausible relationship between the use of this biomarker and CAD risk. It is evident that this biomarker reflects both fatty acid metabolism and cellular composition. However, dietary intake of omega-3 PUFA was not associated with content of omega-3 PUFA in RBC FA profiles of individuals with and at risk for CAD. It may be of interest to evaluate whether dietary intake of other fat types and individual fatty acids are correlated with their respective RBC membrane levels. Surprisingly, oxidative stress was not significantly associated with CAD, which may likely be due to uric acid in fresh human plasma. The assay used in this study to measure total antioxidant capacity may not have been an accurate predictor of oxidative stress levels in both cases and controls.

It is important to note that the data in the present study are preliminary. As a means to reach the entire projected sample size $(n=100)$, data collecting will continue and may further substantiate the results found in the present study. It is intended to correct some of the limitations that were apparent in this study such as using a measure of oxidative stress in which uric acid is not confounded in the results. For instance, future analyses will likely utilize thiobarbituric acid reactive substances (TBARS), which should circumvent confounding effects of uric acid in the plasma and provide a more reliable, quantitative measure of oxidative stress. Previous studies 
have shown relation between elevated levels of TBARS and CVD risk factors in individuals with stable CAD. ${ }^{111}$ This particular evidence suggests that using TBARS as a measure of oxidative stress may have predictive value as a clinical biomarker in evaluating CAD risk as well as the possible need for antioxidants in the treatment of CAD ${ }^{111}$ Some limitations regarding the use of the FFQ will still remain including the dependence on accuracy of participants' memories and willingness to report details of diet. As a means to limit errors or confusion and to help prompt for more accurate responses from participants, a nutrition professional will be present when FFQs are given to participants in the future. This may also help eliminate the difficulty participants were having in remembering the specific brand and dosage of fish oil supplements. Additionally, when a larger sample size is achieved, the use of additional covariates in statistical models can be permitted, which may increase the predictive power of the model by reducing the effect of confounding variables.

The implications of this preliminary research contribute to the field of nutrition and dietetics by demonstrating an opportunity for registered dietitians (RDs) to participate in emerging clinical research evaluating the future use of biomarkers that can easily be assessed by RDs in health care settings. The efforts of this research not only provides an example of nontraditional roles of RDs but also the potential for RDs to advocate their profession, capability and expertise to develop clinical research questions and apply science-based evidence in the clinical setting. Overall, this study succeeded in finding characteristic differences in diet and lifestyle behaviors of individuals with and at risk for CAD. Analysis of RBC membrane FA composition may represent a more reliable measure of fatty acid intake and metabolic processes that can aid in choosing effective dietary intervention therapies based on disease status. Future studies of greater sample size are needed to validate this biomarker as a predictor of CAD in 
individuals who are at risk and may benefit from dietary interventions to modify RBC FA profiles. 


\section{REFERENCES}

1. Mahan KL, Escott-Stump S. Krause's food \& nutrition therapy. 12th ed. St. Louis, Missouri: Saunders, Elsevier Inc.; 2008

2. Tziakas DT, Chalikias GK, Stakos D, Boudoulas H. The role of red blood cells in the progression and instability of atherosclerotic plaque. International Journal of Cardiology. June 2010; 142(1): 2-7.

3. Finn AV, Nakano M, Narula J, Kolodgie FD, Virmani R. Concept of vulnerable/unstable plaque. Arteriosclerosis, Thrombosis, and Vascular Biology. 2010; 30: 1282-1292.

4. Sudheedran S, Chang CC, Deckelbaum RJ. N-3 vs. saturated fatty acids: effects on the arterial wall. Prostaglandins Leukot Essent Fatty Acids. 2010; 82(4-6): 205-209.

5. Felton CV, Crook D, Davies MJ, Oliver MF. Dietary polyunsaturated fatty acids and composition of human aortic plaques. The Lancet. 1994; 344(8931): 1195-6.

6. Lausada NR, Boullon S, Boullon F, Tacconi De Gomez Dumm IN. Erythrocyte membranes, plasma and atherosclerotic plaque lipid pattern in coronary heart disease. Medicina. 2007; 67(5).

7. Jackson JA, Riordan HD, Hunnunghake R, Meng X, Sarwar Y. Red blood cell membrane fatty acids as a diagnostic test. Journal of Othromolecular Medicine. 1997; 12(1): 20-22.

8. Simopoulos, AP. The importance of the omega-6/omega-3 fatty acid ratio in cardiovascular disease and other chronic diseases. Experimental Biology \& Medicine. 2008; 233(6): 674-688.

9. Simopoulos AP. Essential fatty acids in health and chronic disease. The American Journal of Clinical Nutrition. 1999;70(suppl):560S-569S.

10. Calder PC, Grimble RF. Polyunsaturated fatty acids, inflammation and immunity. European Journal of Clinical Nutrition. 2002; 56(3): S14-S19.

11. Sardesai VM. Nutritional role of polyunsaturated fatty acids. J Nutr. Biochem. 1992; 3:154-166.

12. Kris-Etherton PM, Taylor DS, Yu-Poth S, Huth P, Moriarty K, Fishell V, Hargrove RL, Zhao G, Etherton TD. Polyunsaturated fatty acids in the food chain in the United States. Am J Clin Nutr. 2000;71(suppl):179S-188S.

13. Kris- Etherton PM, Harris WS, Appel LJ. Fish consumption, fish oil, omega-3 fatty acids, and cardiovascular disease. Circulation. 2003;106: 2747-2757.

14. Lee KW, Lip GYH. The role of omega-3 fatty acids in secondary prevention of cardiovascular disease. Q J Med. 2003;96: 465-480. 
15. Calder PC. N-3 fatty acids and cardiovascular disease: evidence explained and mechanisms explored. Clinical Science. 2004;107: 1-11.

16. Wang C, Harris WS, Chung M, Lichtenstein AH, Balk EM, Kupelnick B, Jordan HS, Lau J. n-3 fatty acids from fish or fish-oil supplements, but not alpha-linolenic acid, benefit cardiovascular disease outcomes in primary and secondary prevention studies: a systematic review. Am J Clin Nutr. 2006; 84(1): 5-17.

17. Lindberg M, Saltvedt I, Sletvold O, Bjerve KS. Long-chain n-3 fatty acids and mortality in elderly patients. Am J Clin Nutr. 2008; 88:722-9.

18. GISSI-Prevenzione Investigators. Dietary supplementation with n-3 polyunsaturated fatty acids and vitamin E after myocardial infarction: results of the GISSI-Prevenzione trial. Lancet. 1999; 354(9177): 447-455.

19. Marchioli R, Barzi F, Bomb E, Chieffo C, Di Gregorio D, Di Mascio R, Franzosi MG, Geraci E, Levantesi G, Maggioni AP, Mantini L, Marfisi RM, Mastrogiuseppe G, Mininni N, Nicolosi GL, Santini M, Schweiger C, Tavazzi L, Togoni G, Tucci C, Valagussa F. Early protection against sudden death by n-3 polyunsaturated fatty acids after myocardial infarction: time-course analysis of the results of the Gruppo Italiano per lo Studio della Sopravvivenza nell'Infarto Miocardico (GISSI)-Prevenione. Circulation. 2002; 105: 1897-1903

20. Burr ML, Fehily AM, Gilbert JF, Rogers S, Holiday RM, Sweetnam PM, Elwood PC, Deadman NM. Effects of changes in fat, fish, and fiber intakes on death and myocardial reinfarction: Diet and Reinfarction Trial (DART). Lancet. 1989;2: 757-761.

21. Yokoyama M, Origasa H, Matsuzaki M, Matsuzawa Y , Saito Y , Ishikawa Y, et al.. Effects of eicosapentaenoic acid on major coronary events in hypercholesterolaemic patients (JELIS): A randomized open-label, blinded endpoint analysis. Lancet 2007; 369: 1090 - 1098.

22. London B, Albert C, Anderson ME, Giles WR, Van Wagoner DR, Balk E, Billman GE, Chung M, Lands W, Leaf A, McAnulty J, Martens JR, Costello RB, Lanthrop DA. Omega-3 fatty acids and cardiac arrhythmias: prior studies and recommendations for future research: a report from the National Heart, Lung, and Blood Institute and Office of Dietary Supplements Omega-3 Fatty Acids and Their Role in Cardiac Arrhythmogenesis Workshop. Circulation. 2007; 116e: 320e-e335.

23. Nelms M, Sucher KP, Lacey K, Roth SL. Nutrition therapy and pathophysiology: $2^{\text {nd }}$ ed. Wadsworth, Inc. 2011.

24. Harris WS, Sands SA, Windsor SL, Ali HA, Stevens TL, Magalski A, Porter CB, Borkon AM. Omega-3 fatty acids in cardiac biopsies from heart transplantation patients: correlation with erythrocytes and response to supplementation. Circulation. 2004; 110:1645-1649. 
25. Pepe S, McClennan PL. Dietary fish oil confers direct antiarrhythmic properties on the myocardium of rats. J. Nutr. 1996; 126:34-42.

26. McLennan PL, Bridle TM, Abeywardena MY, Charnock JS. Comparative efficacy of n-3 and n-6 polyunsaturated fatty acids in modulating ventricular fibrillation threshold in marmoset monkeys. Am J Clin Nutr. 1993; 58(5): 666-669.

27. Kinoshita I, Itoh K, Nishida-Nakai M, Hirota H, Otsuji S, Shibata N. Antiarrhythmic effects of eicosapentaenoic acid during myocardial infarction - enhanced cardiac microsomal (Ca(2+)-Mg2+)-ATPase activity. Jpn Circ J. 1994;58:903-912.

28. Cheng JWM, Santoni F. Omega-3 fatty acid: a role in the management of cardiac arrhythmias? Journal of Alternative \& Complementary Medicine. 2008;14(8): 965-974.

29. Nair SSD, Leitch JW, Falconer J, Garg ML. Prevention of cardiac arrhythmia by dietary (n-3) polyunsaturated fatty acids and their mechanism of action. $J$ Nutr. 1997;127(3): 383-393.

30. Leaf A, Kang JX, Xiao YF, Billman GE. Clinical prevention of sudden cardiac death by n-3 polyunsaturated fatty acids and mechanism of prevention of arrhythmias by n-3 fish oils. Circulation. 2003; 107: 2646-2652.

31. Kang JX, Leaf A, Prevention of fatal cardiac arrhythmias by polyunsaturated fatty acids. Am J Clin Nutr. 2000;71(suppl): 202S-207S.

32. Xiao YF, Kang JX, Morgan JP, Leaf A. Blocking effects of polyunsaturated fatty acids on $\mathrm{Na}^{+}$channels of neonatal rat ventricular myocytes. Proc Natl Acad Sci USA. 1995;92: 11000-4.

33. Xiao Y-F, Gomez AM, Morgan JP, Lederer WJ, Leaf A. Suppression of voltage-gated Ltype $\mathrm{Ca}^{2+}$ currents by polyunsaturated fatty acids in adult and neonatal rat cardiac myocytes. Proc Natl Acad USA. 1997; 94:4182-7.

34. Christensen JH, Korup E, Aaroe J, Toft E, Moller J, Rasmussen K, Dyerberg J, Schmidt EB. Fish consumption, n-3 fatty acids in cell membranes, and heart rate variability in survivors of myocardial infarction with left ventricular dysfunction. The American Journal of Cardioology. 1997;79(12): 1670-1673.

35. Christensen JH, Christensen MS, Dyerberg J, Schmidt EB. Heart rate variability and fatty acid content of blood cell membranes: a dose response study with n-3 fatty acids. Am J Clin Nutr. 1999;70(3): 331-337.

36. Luostarinena R, Boberg M, Saldeen T. Fatty acid composition in total phospholipids of human coronary arteries in sudden cardiac death. Atherosclerosis. 1993; 99(2): 187-93.

37. Mozaffarian D. Fish and n-3 fatty acids for the prevention of fatal coronary heart disease and sudden cardiac death. Am J Clin Nutr. 2008; 87(suppl): 1991S-6S. 
38. Raitt MH, Connor WE, Morris C, Kron J, Halperin B, Sumeet SC, McClellan J, Cook, J, MacMurdy K, Swenson R, Connor SL, Gerhard G, Kraemer DF, Oseran D, Marchant C, Calhoun D, Schnider R, McAnulty J. Fish oil supplementation and risk of ventricular tachycardia and ventricular fibrillation in patients with implantable defibrillators: a randomized controlled trial. JAMA. 2005;293(23): 2884-2891.

39. Leaf A, Albert CM, Joesphson M, Steinhaur D, Kluger J, Kang JX, Cox B, Zhang H, Schoenfeld D: Fatty Acid Antiarrhythmia Trial Investigators. Prevention of fatal arrhythmias in high-risk subjects by fish oil n-3 fatty acid intake. Circulation. 2005; 112: 2762-2768.

40. Brouwer IA, Zock PL, Camm AJ, Bocker D, Hauer RNW, Wever EFD, Dullemeijer C, Ronden JE, Katan MB, Lubinski A, Buschler H, Schouten EG.; the SOFA Study Group. Effect of fish oil on ventricular tachyarrhythmia and death in patients with implantable cardioverter defibrillators: the study on omega- 3 fatty acids and ventricular arrhythmia (SOFA) randomized trial. JAMA. 2006; 295(22): 2613-2619.

41. Mozaffarian D, Ascherio A, Hu FB, Stampfer MJ, Willett WC, Siscovick DS, Rimm EB. Interplay between different polyunsaturated fatty acids and risks of coronary heart disease. Ciculation. 2005; 111:157-164.

42. Albert CM, Kyungwon O, Whang W, Manson JE, Chae CU, Stampfer MJ, Willett WC, Hu FB. Dietary alpha-linolenic acid intake and risk of sudden cardiac death and coronary heart disease. Circulation. 2005; 112:3232-3238.

43. Ander BP, Weber AR, Rampersad PP, Gilchrist JSC, Pierce GN, Lukas A. Dietary flaxseed protects against ventricular fibrillation induced by ischemia-reperfusion in normal and hypercholesterolemic rabbits. J. Nutr. 2004; 134(12):3250-3256.

44. McClennan PL, Dallimore JA. Dietary canola oil modifies myocardial fatty acids and inhibits cardiac arrhythmias in rats. J. Nutr. 1995; 125:1003-1009.

45. Billman GE, Kang JX, Leaf A. Prevention of sudden cardiac death by dietary pure omega-3 polyunsaturated fatty acids in dogs. Circulation. 1999; 99:2452-2457.

46. Di Lorgeril M, Salen P, Martin JL, Monjaud I, Delaye J, Mamelle N. Mediterranean diet, traditional risk factors, and the rate of cardiovascular complications, after myocardial infarction: final report of the Lyon Diet Heart Study. Circulation. 1999;99: 779-785.

47. Singh RB, Dubnov G, Niaz MA, Ghosh S, Singh R, Rastogi SS, Manor O, Pella D, Berry EM. Effect of an Indo-Mediterranean diet on progression of coronary artery disease in high risk patients (Indo-Mediterranean Diet Heart Study): a randomized single-blind trial. Lancet. 2002; 360(9344): 1455-1461.

48. Benedetto U, Melina G, di Bartolomeo R, Angeloni E, Sansone D, Falaschi G, Capuano F, Comito C, Roscitano A. n-3polyunsaturated fatty acids after coronary artery bypass grafting. Ann Thorac Surg. 2011; 91: 1169-1175. 
49. Eritsland J, Arnesen H, Gronseth K, Fjeld NB, Abdelnoor M. Effect of dietary supplementation with $\mathrm{n}-3$ fatty acids on coronary artery bypass grafts patency. Am $J$ Cardiol. 1996; 77(1); 31-36.

50. Virmani R, Kolodgie FD, Burke AP, Finn AV, Gold HK, Tulenko TN, Wrenn SP, Narula $\mathrm{J}$. Atherosclerotic plaque progression and vulnerability to rupture: angiogenesis as a source of intraplaque hemorrhage. Arterioscler Thromb Vasc Biol. 2005; 25: 2054-2061.

51. Davis HR, Bridenstine RT, Vesselinovitch D, Wissler RW. Fish oil inhibits development of atherosclerosis in rhesus monkeys. Arteriosclerosis. 1987; 7: 441-9.

52. Weiner BH, Ockene IS, Levine PH, Vaudreuil C, Hoogasian JJ. Inhibition of atherosclerosis by cod liver oil in a hyperlipidaemic swine model. $N$ Engl J Med. 1986; 315: 841-6.

53. Mortensen A, Fischer Hansen B, Fisher Hansen J, et al.. Comparison of the effects of fish oil and olive oil on blood lipids and aortic atherosclerosis in Wantanabe heritable hyperlipidaemic rabbits. Br J Nutr. 1998; 80: 565-73.

54. Grimble RF. Dietary lipids and the inflammatory response. Proc Nutr Soc. 1998; 57: 53542.

55. Rapp JH, Connor WE, Lin DS, Porter JM. Dietary eicosapentanoic acid and docosahexanoic acid from fish oil. Their incorporation into advanced human atherosclerotic plaques. Atheroscler Thromb Vasc Biol. 1991; 11: 903-911.

56. Small DM, Shipley GG. Physical chemical basis of lipid deposition in atherosclerosis: the physical state of lipids helps to explain lipid deposition and lesion reversal in atherosclerosis. Science. 1975; 185: 222-29.

57. Singh RB, Niaz MA, Sharma JP, et al.. Randomized double blind, placebo-controlled trial of fish oil and mustard oil in patients with suspected acute myocardial infarction: the Indian experiment of infarct survival. Cardiovasc Drugs Ther. 1997; 11: 85-91.

58. Thies F, Garry JCM, Yaqoob P, Rerkasem K, Williams J, Shearman CP, Gallagher PJ. Association of n-3 polyunsaturated fatty acids with stability of atherosclerotic plaques: a randomized controlled trial. Lancet. 2003; 361: 477-85.

59. Schumacher H, Kaiser E, Schnabel PA, Sykora J, Eckstein HH, Allenberg JR. Immunophenotypic characterization of carotid plaque: increased amount of inflammatory cells as an independent predictor for ischaemic symptoms. Eur J Vasc Endovasc Surg. 2001; 21: 494-501.

60. Kolodgie FD, Burke AP, Nakazawa G, Cheng Q, Xu Z, Virmani R. Free cholesterol in atherosclerotic plaques: where does it come from? Curr Opin Lipidol. 2007; 18: 500-7.

61. Kolodgie FD, Gold HK, Burke AP, et al.. Intraplaque hemorrhage and progression of coronary atheroma. $N$ Engl J Med. 2003; 349: 2316-25. 
62. Katz SS, Shipley GG, Small DM. Physical chemistry of the lipids of human atherosclerotic lesions. Demonstration of a lesion intermediate between fatty streaks and advanced plaques. J Clin Invest. 1976; 58: 200-11.

63. Cawood AL, Ding R, Napper FL, Young RH, Williams JA, Ward MJA, Gudmundsen O, Vige R, Payne SPK, Ye S, Shearman CP, Gallagher PJ, Grimble RF, Calder PC. Eicosapentanoic acid (EPA) from highly concentrated n-3 fatty acid ethyl esters is incorporated into advanced atherosclerotic plaques and higher plaque EPA is associated with decreased plaque inflammation and increased instability. Atheroscolerosis. 2010; 212(1): 252-9.

64. Sun Q, Ma J, Campos H, Hankinson SE, Hu FB. Comparison between plasma and erythrocyte fatty acid content as biomarkers of fatty acid intake in US women. $J$ Clin Nutr. 2007; 86: 74-81.

65. Albert CM, Campos H, Stampfer MJ, Ridker PM, Manson JE, Willet WC, Ma J. Blood levels of long-chain n-3 fatty acids and the risk of sudden death. $N$ Engl $J$ Med. 2002; 346(15): 1113-1118.

66. Block RC, Harris WS, Reid KJ, Sands SA, Spertus JA. EPA and DHA in blood cell membranes from acute coronary syndrome patients and controls. Atherosclerosis. 2008; 197: 821-828.

67. Wilson PW, D’Agostino RB, Levy D, Belanger AM, Silbershatz H, et al.. Prediction of coronary heart disease using risk factor categories. Circulation. 1998; 97: 1937-1847.

68. Shearer GC, Pottala JV, Spertus JA, Harris WS. Red blood cell fatty acid patterns and acute coronary syndrome. PLOS ONE. 2009; 4(5): e5444.

69. Park Y, Lim J, Lee J, Kim S. Erythrocyte fatty acid profiles can predict acute non-fatal myocardial infarction. British Journal of Nutrition. 2009; 102: 1355-1361.

70. Harris WS. The omega-3 index as a risk factor for coronary heart disease. Am J Clin Nutr. 2008; 87: 1997S-2002S.

71. Harris WS \& von Shacky C. The omega-3 index: a new risk factor for death from coronary heart disease? Prev Med. 2004; 39: 212-20.

72. Harris WS, Sands SA, Windsor SL, Ali HA, Stevens TL, Magalski A, Porter CD, Borkon AM. Omega-3 fatty acids in cardiac biopsies from heart transplantation patients: correlation with erythrocytes and response to supplementation. Circulation. 2004; 110: 1645-1649.

73. Siscovick DS, Raghunathan TE, King I, Weinmann S, Wisklund KG, Albright J, Bovbjerg V, Arbogast P, Smith H, Kushi LH, Cobb LA, Copass MK, Psaty BM, Lemaitre R, Retzlaff B, Childs M, Knopp RH. Dietary intake and cell membrane levels of long-chain n-3 polyunsaturated fatty acids and the risk of primary cardiac arrest. JAMA. 1995; 274: 1363-1367. 
74. Aarestoey H, Ponitz V, Grundt H, Staines H, Harris WS, Nilsen DWT. (n-3) fatty acid content of red blood cells does not predict risk of future cardiovascular events following an acute coronary syndrome. J. Nutr. 2009; 139: 507-513.

75. Harris WS, Kennedy KF, O’Keefe Jr. JH, Spertus JA. Red blood cell fatty acid levels improve GRACE score prediction of 2-yr mortality in patients with myocardial infarction. Int J Cardiol. 2013; 168(1): 53-39.

76. Lands WE. Long-term fat intake and biomarkers. Am J Clin Nutr. 1995; 61: 721S-725S.

77. Sands SA, Reid KJ, Windsor SL, Harris WS. The impact of age, body mass index, and fish intake on the EPA and DHA content of human erythrocytes. Lipids. 2005; 40(4): 343-347.

78. Itomura M, Fujioka S, Hamazaki K, Kobayashi K, Nagasawa T, Sawazaki S, Kirihara Y, Hamazaki T. Factors influencing EPA+DHA levels in red blood cells in Japan. In vivo. 2008; 22: 131-136.

79. Gudbyarnason S. Dynamics of n-3 and n-6 fatty acids in phospholipids of heart muscle. $J$ Intern Med. 1989; 225(Suppl. 1): 117-128.

80. Rise P, Elgini S, Ghezzi S, Colli S, Galli C. Fatty acid composition if plasma, blood cells and whole blood: relevance for the assessment of the fatty acid status in humans. Prostaglandins, Leukotrienes, and Essential Fatty Acids. 2007; 76: 363-369.

81. Metcalf RG, Cleland LG, Gibson RA, Roberts-Thomson KC, Edwards JRM, Sanders P, Stuklis R, James MJ, Young GD. Relation between blood and atrial fatty acids in patients undergoing cardiac bypass surgery. Am J Clin Nutr. 2010; 91: 528-534.

82. Sublette ME, Segal-Issacson CJ, Cooper TB, Fekri S, Vanegas N, Galfalvy HC, Oquendo A, Mann J. Validation of a food frequency questionnaire to assess intake of n-3 polyunsaturated fatty acids with and without major depressive disorder. J Am Diet Assoc. 2011; 111: 117-123.

83. Benzie IFF, Strain JJ. The ferric reducing ability of plasma (FRAP) as a measure of "antioxidant power”: the FRAP assay. Analytical Biochemistry. 1996; 239: 70-76.

84. Cazzola R, Rondanelli M, Russo-Volpe S, Ferrari E, Cestaro B. Decreased membrane fluidity and altered susceptibility to peroxidation and lipid composition in overweight and obese female erythrocytes. J Lipid Res. 2004; 45: 1846-1851.

85. Keaney JF, Larson MG, Vasan RS, Wilson PWF, Lipinska I, Corey D, Massaro JM, Sutherland P, Vita JA, Benjamin EJ. Obesity and systemic oxidative stress: clinical correlates of oxidative stress in the Framingham study. Arterioscler Thromb Vasc Biol. 2003; 23: 434-439. 
86. Furukawa S, Fujita T, Shimabukuro M, Iwaki M, Yamada Y, Nakajima Y, Nakayama O, Makishima M, Matsuda M, Shimomura I. Increased oxidative stress in obesity and its impact on metabolic syndrome. J Clin Invest. 2004; 114(12): 1752-61.

87. Morrow JD, Frei B, Longmire AW, Gaziano M, Lynch SM, Shyr Y, Strauss WE, Oates JA, Roberts LJ. Increase in circulating products of lipid peroxidation ( $\mathrm{F}_{2}$-isoprostanes) in smokers: smoking as a cause of oxidative damage. N Engl J Med. 1995; 332: 1198-1203.

88. Rizvi SI, Jha R, Maurya PK. Erythrocyte plasma membrane redox system in human aging. Rejuvenation Research. 2006; 9(4): 470-474.

89. Hirata Y, Satonaka H. Hypertension and oxidative stress. JMAJ. 2001; 44(12): 540-45.

90. Ward NC, Croft KD. Hypertension and oxidative stress. Clin. Exp. Pharmacol. Physiol. 2006; 33: 872-76.

91. Alexander JK. Obesity and coronary heart disease. Am J Med Sci. 2001; 321(4): 215-224.

92. Leon AS, Bronas UG. Dyslipidemia and risk of coronary heart disease: role of lifestyle approaches for its management. Am J Lifestyle Med. 2009; 3(4): 257-73.

93. Matsumoto C, Matthan NR, Lichtensten AH, Gaziano JM, Djousse L. Red blood cell MUFAs and risk of coronary artery disease in the Physicians' Health Study. Am J Clin Nutr. 2013; 98: 749-54.

94. Siri-Tarino PW, Sun Qi, Krauss RM. Saturated fatty acids and risk of coronary heart disease: modulation by replacement nutrients. Curr Atheroscler Rep. 2010; 12(6): 38490 .

95. Mente A, de Koning L, Shannon HS, Anand SS. A systematic review of the evidence supporting a casual link between dietary factors and coronary heart disease. Arch Intern Med. 2009; 169(7): 659-669.

96. Hu FB, Stampfer MJ, Manson JE, et al..: Dietary saturated fats and their food sources in relation to the risk of coronary heart disease in women. Am J Clin Nutr 1999, 70:10011008

97. de Lorgeril M, Renaud S, Mamelle N, et al.. Mediterranean alpha-linolenic acid-rich diet in secondary prevention of coronary heart dis - Ease. L ancet $1994 ; 343: 1454$

98. Harris WS, Mozaffarian D, Rimm E, Kris-Etherton P, Rudel LL, Appel LJ, Engler MM, Engler MB, Sacks F. Omega-6 fatty acids and risk for cardiovascular disease: a science advisory from the American Heart Association Nutrition Subcommittee of the Council on Nutrition, Physical Activity and Metabolism; Council on Cardiovascular Nursing; and Council on Epidemiology and Prevention. Circulation. 2009; 119: 902-907.

99. Olsen SF, Hansen HS, Sanstrom N, Jensen B. Erythrocyte levels compared with reported dietary intake of marine n-3 fatty acids in pregnant women. Br J Nutr. 1995; 73: 387-395. 
100. Parra M, Schnass L, Meydani M, Perroni E, Martianez S, Romieu I. Erythrocyte cell membrane phospholipid levels compared against reported dietary intakes of polyunsaturated fatty acids in pregnant Mexican women. Public Health Nutr. 2002; 5: 931-37. Food and Drug Administration: Substance affirmed as generally recognized as safe: menhaden oil. Fed Regist. 1997; 30751-30757.

101. Harsem NK, Braekke K, Staff AC. Augmented oxidative stress as well as antioxidant capacity in maternal circulation in preeclampsia. Eur J Obset Gynecol Reprod Biol. 2006; 128: 209-215.

102. World Health Organization (WHO). Global Atlas on Cardiovascular Disease Prevention and Control. Geneva, Switzerland: WHO; 2011.

103. Lloyd-Jones DM, Hong Y, Labarthe D, et al. Defining and setting national goals for cardiovascular health promotion and disease reduction: the American Heart Association's Strategic Impact Goal through 2020 and beyond. Circulation. 2010;121(4):586-613.

104. Ahmed HM, Blaha MJ, Nasir K, et al. Low-risk lifestyle, coronary calcium, cardiovascular events, and mortality: results from multi-ethnic study of atherosclerosis (MESA). Am J Epidemiol. 2013;178(1):12-21.

105. Chiuve SE, Fung TT, Rexrode KM, et al. Adherence to a low-risk, healthy lifestyle and risk of sudden cardiac death among women. JAMA. 2011;306(1):62-69.

106. Lodovici M, Bigagli E, Bardini G, Rotella CM. Lipoperoxidation and antioxidant capacity in patients with poorly controlled type 2 diabetes. Toxicol Ind Health. 2009;25:337-341.

107. Hetyey CS, Manczur F, Dudas-Gyorki Z, Reiczigel J, Ribiczey P, Vajdovich P, Voros K. Plasma antioxidant capacity in dogs with naturally occurring heart diseases.J Vet Med. 2007;54: 36-39.

108. Shirvani SS, Rasmi Y, Seyyed-Mohammadzad MH, Khosravifar F. Oxidative stress status in patients with cardiac syndrome X. ScienceAsia. 2012;38:136-140.

109. Harma MI \& Erel O. Are d-ROMs and FRAP tests suitable assays for detecting the oxidative status? Letters to the Editor/ Eur J Obset Gynecol Reprod Biol. 2006;127

110. Libby P. Inflammtion and cardiovascular disease mechanisms. Am J Clin Nutr. 2006;83: 456S-460S.: 271-275.

111. Walter MF, Jacob RF, Jeffers B, et al. Serum levels of thiobarbituric acid reactive substances predict cardiovascular events in patients with stable coronary artery disease: a longitudinal analysis of the PREVENT study. J Am Coll Cardiol. 2004;44(10):19962002. 


\title{
APPENDICES
}

\author{
APPENDIX A: IRB Approval \\ East Tennessee State University \\ Office for the Protection of Human Research Subjects • Box 70565 • Johnson City, Tennessee 37614-1707 \\ Phone: (423) 439-6053 Fax: (423) 439-6060
}

IRB APPROVAL - Initial Expedited Review

April 9, 2013

Dr. Andrew Clark

Box 70552

Re: Assessment of red blood cell membrane fatty acid composition in relation to dietary intake in patients with coronary artery disease

IRB\#:0313.35s

ORSPA \#:

The following items were reviewed and approved by an expedited process:

- New Protocol submission form, ICD version 3/19/2013, OMega 3 Fatty Acid Questionnaire, Supplemental Questionnarie, CV

On April 9, 2013, a final approval was granted for a period not to exceed 12 months and will expire on April 8, 2014. The expedited approval of the study will be reported to the convened board on the next agenda.

The following enclosed stamped, approved Informed Consent Documents have been stamped with the approval and expiration date and these documents must be copied and provided to each participant prior to participant enrollment:

- ICD version $3 / 19 / 2013$ stamped $4 / 9 / 2013$

Federal regulations require that the original copy of the participant's consent be maintained in the principal investigator's files and that a copy is given to the subject at the time of consent.

\section{Projects involving Mountain States Health Alliance must also be approved by MSHA following IRB approval prior to initiating the study.}

Unanticipated Problems Involving Risks to Subjects or Others must be reported to the IRB (and VA R\&D if applicable) within 10 working days.

Proposed changes in approved research cannot be initiated without IRB review and approval. The only exception to this rule is that a change can be made prior to IRB approval when necessary to

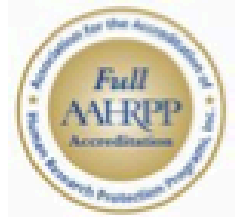

Ancredited Sisce December 2005 
eliminate apparent immediate hazards to the research subjects [21 CFR 56.108 (a)(4)]. In such a case, the IRB must be promptly informed of the change following its implementation (within 10 working days) on Form 109 (www.etsu.edu/irb). The IRB will review the change to determine that it is consistent with ensuring the subject's continued welfare.

Sincerely,

George Youngberg, M.D., Chair

ETSU/VA Medical IRB 


\title{
APPENDIX B: Permission Letter to Use Food Frequency Questionnaire
}

\author{
M. Elizabeth Sublette \\ Division of Molecular Imaging and Neuropathology \\ New York State Psychiatric Institute \\ 1051 Riverside Drive, Unit 42, Room 2711 \\ NY, NY 10032
}

Dear De. Sublette,

This letter will confirm out recent email exchange on November 26,2012. I am completing a master's thesis at East Tennessee State University entitled "Assessment of red blood cell membrane fatty acid composition in relation to dietary intake in patients 1Jlldergoing cardiac catheterization." I would like your permission in my thesis for utilization of your food frequency questionnaire in JAm Diet Assac. 2011; 111: 117-123. My intentions of using your instrument in my thesis are as follows:

- to assess omega-3 fatty acid consumption, and

- for correlation ofreported omega-3 fatty acid intake and omega-3 fatty acid content of the red blood cell membrane.

The requester permission extends to any future revisions and editions of my thesis, including noa-exclusive world rights in all languages, and to the prospective publication of my thesis by UMI. These rights will in no way restrict publication of the material in any other form by you or by others authorized by you. Your signing of this letter will also confirm that you own the copyright to the food frequency questionnaire described above.

If these arrangements meet with you approval, please sign this letter where indicated below. Thank you very much.

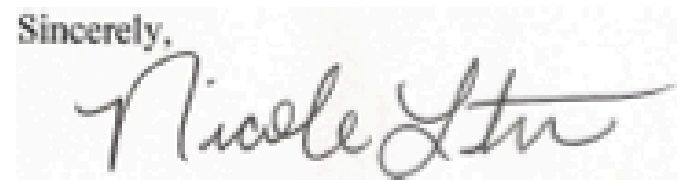

Nicole Litwin

PERMISSION GRANTED FOR THE USE REQUESTED ABOVE<smiles>CCCCCCCCCCCCC</smiles>

M. Elizabeth Sublette

Division of Molecular Imaging and Neuropathology

New York State Psychiatric Institute

1051 Riverside Drive, Unit 42, Room 2711

NY, NY 10032 
APPENDIX C: Omega-3 Fatty Acid Food Frequency Questionnaire

\section{Omega 3 Fatty Acid Questionnaire}

1) Gender: $\square$ Male (1) $\square$ Female (2)

2) About how long ago was your last meal?

hours

3) Have you eaten any fish or shellfish--such as shrimp, crab, lobster etc.-in the past 24 hours? (include sushi made with fish or shellfish)

1) $\square$ No

2) $\square$ Yes

4) How many times have you eaten fish or shellfish in the past week?
1) $\square 0$ times
2) $\square 1-3$ times
3) $\square$ More than 3 times

5) Over the past 6 months, about how often have you eaten fish or shellfish in any form?
1) $\square$ Never
6) $\square 2$ times each week
2) $\square$ Less than 1 time each month
7) $\square$ 3-4 times each week
3) $\square 1$ time each month
4) $\square 2-3$ times each month
8) प 5-6 times each week
5) $\square 1$ time each week
9) $\square 1$ time each day
10) $\square 2$ or more times each day

\section{If NEVER, Skip to Question 8.}

6) Each time you ate fish or shellfish, how much did you eat?

1) $\square$ Less than 2 ounces or less than one fillet or less than 4 pieces of sushi

2) $\square 2$ to 7 ounces or about 1 fillet or $4-14$ pieces of sushi

3) $\square$ More than 7 ounces or more than 1 fillet or more than 14 pieces of sushi

7) Please check off the types of fish or shellfish you eat most frequently (Check off as many as are appropriate for you).
1) $\square$ Bass
18) $\square$ Sardines
2) $\square$ Bluefish
19) $\square$ Scallops
3) $\square$ Catfish
4) ロ Clams
5) $\square \mathrm{Cod}$
6) $\square \mathrm{Crab}$
7) $\square$ Flounder
20) $\square$ Sea Trout
21) $\square$ Shark
22) $\square$ Shrimp
23) 口 Skate
8) $\square$ Haddock
24) $\square$ Snapper
9) $\square$ Halibut
10) $\square$ Herring
25) $\square$ Sole
26) $\square$ Swordfish
27) $\square$ Tilapia
11) $\square$ Kingfish
28) $\square$ Tilefish
12) $\square$ Lobster
29) $\square$ Trout (freshwater)
13) $\square$ Mackerel
30) ם Tuna
14) $\square$ Mahi Mahi
31) $\square$ Turbot
15) Mussels
32) $\square$ Whitefish
16) $\square$ Oysters
17) $\square$ Salmon
33) $\square$ Whiting 
8) In the past 6 months, about how often did you eat walnuts?
1) $\square$ Never
6) $\square 2$ times each week
2) $\square$ Less than 1 time each month
7) ㄱ-4 times each week
3) $\square 1$ time each month
8) प 5-6 times each week
4) $\square 2-3$ times each month
9) $\square 1$ time each day
5) $\square 1$ time each week
10) $\square 2$ or more times each day

\section{If NEVER, Skip to Question 10.}

9) Each time you ate walnuts, how much did you eat?
1) $\square$ Less than $1 / 4$ cup
2) $\square \frac{1}{4}$ to $1 / 2$ cup
3) $\square$ More than $1 / 2$ cup

10) In the past 6 months, about how often did you use canola cooking oil?
1) $\square$ Never
2) Less than 1 time each month
3) $\square 1$ time each month
4) $\square$ 2-3 times each month
5) $\square 1$ time each week
6) $\square 2$ times each week
7) $\square$ 3-4 times each week
8) प 5-6 times each week
9) $\square 1$ time each day
10) $\square 2$ or more times each day

\section{If NEVER, Skip to Question 12.}

11) Each time you used canola cooking oil, how much did you use?

1) $\square$ Less than 1 teaspoon

2) $\square$ 1-2 teaspoons

3) $\square 2$ teaspoons

4) $\square 3$ teaspoons ( 1 tablespoon)

5) $\square$ More than 1 tablespoon

12) In the past 6 months, about how often did you eat flaxseed?
1) $\square$ Never
6) $\square 2$ times each week
2) $\square$ less than 1 time each month
7) $\square$ 3-4 times each week
3) $\square 1$ time each month
8) प5-6 times each week
4) $\square$ 2-3 times each month
9) $\square 1$ time each day
5) $\square 1$ time each week
10) $\square 2$ or more times each day

\section{If NEVER, Skip to Question 14}

13) Each time you ate flaxseeds, about how much did you eat?

1) $\square$ Less than 1 teaspoon

2) $\square$ 1-2 teaspoons

3) $\square 2$ teaspoons

4) $\square 3$ teaspoons ( 1 tablespoon)

5) $\square$ More than 1 tablespoon 
14) In the past 6 months, about how often did you use flaxseed oil?
1) $\square$ Never
6) $\square 2$ times each week
2) $\square$ Less than 1 time each month
7) प 3-4 times each week
3) $\square 1$ time each month
4) $\square$ 2-3 times each month
8) $\square$ 5-6 times each week
5) $\square 1$ time each week
9) $\square 1$ time each day
10) $\square 2$ or more times each day

If NEVER, Skip to Question 16.

15) Each time you used flaxseed oil, how much did you have?
1) $\square$ Less than 1 teaspoon
2) $\square$ 1-2 teaspoons
3) $\square 2$ teaspoons
4) $\square 3$ teaspoons ( 1 tablespoon)
5) $\square$ More than 1 tablespoon

16) In the past 6 months, about how often did you use cod liver oil?
1) $\square$ Never
6) $\square 2$ times each week
2) Less than 1 time each month
7) $\square$ 3-4 times each week
3) $\square 1$ time each month
8) $\square$ 5-6 times each week
4) 2-3 times each month
9) $\square 1$ time each day
5) $\square 1$ time each week
10) $\square 2$ or more times each day

If NEVER, Skip to Question 18.

17) Each time you used cod liver oil, how much did you have?
1) $\square$ Less than 1 teaspoon
2) $\square$ 1-2 teaspoons
3) $\square 2$ teaspoons
4) $\square 3$ teaspoons (1 tablespoon)
5) $\square$ More than 1 tablespoon

18) In the past 6 months, have you used an Omega 3 fatty acids or fish oil supplement at least once each week?

NO (YOU ARE FINISHED WITH THIS QUESTIONNAIRE)

$\square$ YES - What type of an Omega 3 fatty acids or fish oil supplement do you take?

Please write the name of the supplement below: 
19) Is the Omega 3 fatty acids or fish oil supplement in pill or capsule form?

$\square$ NO (Skip to Question 20)

$\square$ YES - How many did you take?

1) $\square 1$ pill or capsule each week

5) $\square 1$ pill or capsule each day

2) $\square 2$ pills or capsules each week

6) $\square 2$ pills or capsules each day

3) $\square$ 3-4 pills or capsules each week

7) $\square$ 3-4 pills or capsules each day

4) $\square$ 5-6 pills or capsules each week

8) $\square 5$ or more pills or capsules each day

20) Is the Omega 3 fatty acids or fish oil supplement (besides cod liver oil) in liquid form?

$\square$ NO

GYES - How much did you take?

1. $\square$ Less than 1 tablespoon each week

6. $\square 1$ tablespoon each day

2. $\square 1$ tablespoon each week

7. $\square 2$ tablespoons each day

3. $\square 2$ tablespoons each week

8. 3-4 tablespoons each day

4. 3-4 tablespoons each week

5. $\square$ 5-6 tablespoons each week

9. $\square 5$ or more tablespoons each day

21) Please write down the dosage of Omega 3 fatty acids or fish oil supplement, if you know it:

\section{Dosage:}

$\square$ Pills Or Capsules: Mg Per Pill Or Capsule

$\checkmark$ Liquid: $\square$ Don't know dosage Mg Per Tablespoon 
APPENDIX D: Results from General Linear Models

Table 1B: Results of general linear models (GLM) predicting individual fatty acid levels by control and case status

\begin{tabular}{|l|l|l|l|}
\hline Fatty Acids & $\boldsymbol{F}$-Ratio & $\boldsymbol{d} \boldsymbol{f}$ & $\boldsymbol{p}$-value \\
\hline Myristic 16:0 & 0.481 & 1 & 0.498 \\
\hline Palmitic 16:0 & 6.091 & 1 & $0.018^{*}$ \\
\hline Palmitoleic 16:1 & 1.960 & 1 & 0.171 \\
\hline Stearic, 18:0 & 0.705 & 1 & 0.407 \\
\hline Oleic, 18:1 & 0.793 & 1 & 0.379 \\
\hline Linoleic, 18:2 & 0.815 & 1 & 0.373 \\
\hline DGLA, 20:3 & 0.445 & 1 & 0.626 \\
\hline Arachidonic, 20:4 & 0.590 & 1 & 0.448 \\
\hline Adrenic, 22:4 & 1.137 & 1 & 0.294 \\
\hline DPA, 22:5, $\mathrm{n}-3$ & 3.132 & 1 & 0.151 \\
\hline DHA, 22:6 & 0.139 & 1 & 0.719 \\
\hline
\end{tabular}

Table 2B: Results from GLMs predicting average fatty acid type and fatty acid ratios by case status adjusting for BMI and dyslipidemia as covariates. Statistics for the combined corrected model are reported.

${ }^{*} p$-value is significant at $<0.05$

\begin{tabular}{|r|r|r|r|}
\hline Fatty Acid Type/Ratio & F-Ratio & $\boldsymbol{d}$ f & $\boldsymbol{p}$-value \\
\hline Saturated & 0.097 & 3 & 0.961 \\
\hline n-9 PUFA & 2.361 & 3 & 0.089 \\
\hline n-7 PUFA & 1.634 & 3 & 0.200 \\
\hline n-6 PUFA & 4.952 & 3 & $0.006^{*}$ \\
\hline n-3 PUFA & 0.702 & 3 & 0.557 \\
\hline Saturated: Unsaturated & 0.338 & 3 & 0.798 \\
\hline MUFA: PUFA & 0875 & 3 & 0.464 \\
\hline n-6: n-3 & 0.662 & 3 & 0.581 \\
\hline
\end{tabular}

Table 3B: Results of GLM predicting FRAP value by case status

\begin{tabular}{|r|rr|r|rr|}
\hline & F-ratio & df & p-value & \\
\hline FRAP & & 1.033 & & 3 & 0.390 \\
\hline
\end{tabular}




\section{VITA}

\section{NICOLE S. LITWIN}

Education:

Academic \&

Research Experience:

Honors \& Awards:
Cookeville High School, Cookeville, TN 2008

B.S. Allied Health Sciences, Nutrition and Foods Concentration, East Tennessee State University, Johnson City, Tennessee 2012

M.S. Clinical Nutrition \& Dietetic Intern, East Tennessee State University, Johnson City, Tennessee 2014

Graduate Assistant, East Tennessee State University, College of Clinical and Rehabilitative Health Sciences, $2012-2014$

Appalachian Student Research Forum, East Tennessee State University, 2013

Poster presentation on “Assessment of red blood cell membrane fatty acid composition in relation to dietary intake in patients undergoing cardiac catheterization".

Food \& Nutrition Conference Expo, Houston, Texas, 2013

Research \& Practice Innovations poster presentation on “Correlation between motivational factors, psychological barriers and body mass index (BMI) among ETSU college students”

ETSU School of Graduate Studies and Graduate Council Research Grant Recipient, March 2013 (\$739.56)

ETSU School of Graduate Studies Thesis Scholarship, November 2013 